\title{
Total scattering and the pair distribution function in crystallography
}

\author{
David A Keen
}

\section{Published version information}

Citation: DA Keen. 'Total scattering and the pair distribution function in crystallography.' Crystallography Reviews, vol.26, no.3 (2020): 143-201.

DOI: $\underline{10.1080 / 0889311 X .2020 .1797708}$

This is an Accepted Manuscript of an article published by Taylor \& Francis in Crystallography Reviews on $27^{\text {th }}$ August 2020, available online at DOI above.

This version is made available in accordance with publisher policies. Please cite only the published version using the reference above. This is the citation assigned by the publisher at the time of issuing the AAM. Please check the publisher's website for any updates. 


\title{
ARTICLE TEMPLATE
}

\section{Total Scattering and the Pair Distribution Function in Crystallography}

David A. Keen ${ }^{\mathrm{a}}$

a ISIS Facility, Rutherford Appleton Laboratory, Harwell Campus, Didcot, Oxfordshire OX11 OQX, UK

\section{ARTICLE HISTORY}

Compiled July 7, 2020

\begin{abstract}
The importance of total scattering and its Fourier transform, the pair distribution function, in crystallography was understood soon after it was shown that crystals diffract X-rays. However for the next fifty years or so other techniques based more firmly on the crystal lattice came to the fore and total scattering measurements were largely the domain of those studying liquid and amorphous structure. In the late 1980s it was 'rediscovered' as a way to uncover disorder within crystal structures and since then the combination of improved instrumentation and analysis has led to a resurgence of the total scattering method. This review sets out this journey, highlighting both the early origins and-more importantly - the ways that total scattering and pair distribution functions are routinely used within crystallography today.
\end{abstract}

\section{KEYWORDS}

total scattering, pair distribution function (PDF), neutron diffraction, X-ray diffraction, reverse Monte Carlo (RMC), PDFgui, structural disorder, nanocrystals.

PACS CLASSIFICATION

(If needed)

\section{Contents}

1 Introduction 2

2 Historical Perspective 3

2.1 The Early Years $(1912-1927) \ldots \ldots \ldots \ldots$

2.2 Following Zernike and Prins $(1930 \mathrm{~s}) \ldots \ldots \ldots$. . . . . . . . . 5

2.3 The Intervening Years $(1940-1985) \ldots \ldots$. . . . . . . . . . 7

2.3.1 Franklin's study of carbons . . . . . . . . . . . . . 8

$\begin{array}{lll}\text { 2.3.2 Structural studies of metal powders and selenium-based glasses } & 8\end{array}$

2.3.3 Neutron total scattering from liquids and glasses . . . . . . . 11 
3 The PDF in Crystallography Today 12

3.1 Modern Developments . . . . . . . . . . . . . . . . . . . . . 12

3.2 Formalism . . . . . . . . . . . . . . . . . . . . . . 12

3.2.1 Impact of limited total scattering data . . . . . . . . . . . 15

3.3 PDF Instrumentation . . . . . . . . . . . . . . . . . . 15

3.3.1 Neutron total scattering diffractometers . . . . . . . . . 15

3.3.2 X-ray total scattering diffractometers . . . . . . . . . 17

3.4 PDF Data Analysis and Modelling . . . . . . . . . . . . . . . 19

3.4.1 'Big box' modelling and RMCProfile . . . . . . . . . . . . . 19

3.4.2 'Small box' refinements and PDFgui . . . . . . . . . . . . 26

3.5 Magnetic Total Scattering . . . . . . . . . . . . . . . . . 27

3.6 Choosing the optimal radiation/instrumentation/analysis methodology 31

3.6.1 X-ray or neutron total scattering? . . . . . . . . . . . 31

3.6.2 'Big'- or 'small'-box refinement? . . . . . . . . . . . . . . . 32

3.6.3 Hydrogen disorder in hydrogrossular . . . . . . . . . . . . . 33

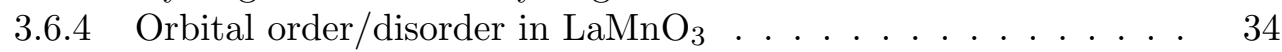

4 A Few Recent Examples $\quad 37$

4.1 Phase transitions . . . . . . . . . . . . . . . . . . 37

4.1 .1 Perovskites . . . . . . . . . . . . . . . . 37

4.1 .2 Solid state amorphization . . . . . . . . . . . . . 39

4.2 Nanoparticles . . . . . . . . . . . . . . . . . . . . . . . . 41

4.2 .1 Introductory remarks $\ldots \ldots \ldots 41$

4.2 .2 'Magic-sized' gold metal clusters . . . . . . . . . . . . . . 42

4.2 .3 Titania nanorods . . . . . . . . . . . . . . . . . 42

4.2 .4 Impact of low- $Q$ scattering . . . . . . . . . . . . . . 43

5 Perspectives and future opportunities $\quad \mathbf{4 4}$

5.1 Future instrumentation . . . . . . . . . . . . . . . . 45

5.2 Future software . . . . . . . . . . . . . . . . . . . 45

5.3 Future directions . . . . . . . . . . . . . . . . 46

\section{Introduction}

One year after the first paper showing X-ray diffraction from single crystals [1], Friedrich measured broad rings of X-ray scattering from non-crystalline substances such as amber, paraffin oil and wax [2]. Friedrich's observations, in part, prompted Debye to develop his eponymous scattering equation [3] and the formalism for total scattering soon followed. Crucially the state of the scattering object, provided it scatters isotropically, is immaterial; these equations are equally applicable to scattering from a crystalline powder, an amorphous material, a liquid or gas. As will be discussed later, this universality of application unhindered by any requirement for 'good' crystallinity is a great strength of total scattering; it has highlighted the significance of local structure in the behaviour of crystalline materials and helped understand the relationship between crystalline, nanocrystalline and amorphous structure.

Following the early groundwork, it became relatively rare for total scattering and the Debye scattering equation to be used within crystallography. The available instrumentation severely limited the resolution of the method and other techniques using single crystal samples and analysis of Bragg scattering alone were able to achieve more 
accurate crystal structures far more straightforwardly. Total scattering was therefore 'relegated' to diffraction studies of materials that didn't yield Bragg peaks. This is not to say that there weren't further developments, but rather that these were largely aimed at reducing the paucity of structural information available from scattering from liquid and amorphous materials.

A resurgence began in the late 1980s through a combination of improvements in instrumentation and computational power and a realisation that our understanding of many crystal structures might be deepened by considering the behaviour of the structure at the local level. Neutron diffraction-particularly at spallation neutron sources - and new refinement tools initiated this renewed use of total scattering, but the community really grew through the transfer of expertise back to the X-ray scattering community as high-energy diffraction became more readily available at synchrotron X-ray sources. It is this era that this review will concentrate on; describing the instrumentation, formalism and methods for interpretation, and highlighting the range of crystalline materials that might benefit from structural investigations using total scattering.

I would like to finish these introductory remarks by stating what total scattering is. In the crystalline context it is all the scattering from the sample, both Bragg and diffuse scattering, which might typically be collected during a carefully taken powder diffraction measurement. Ideally the measurement integrates over all changes of energy within the sample (incorporating scattering from phonons and other dynamic processes) at a constant momentum transfer. Formally this equates to a 'snap-shot' measurement of the sample's structure rather than one with temporal and/or spatial averaging (which is obtained by considering only the elastic scattering, for example). The Fourier transform of the total scattering gives the weighted probability that atoms might be found at certain distances from other atoms, often called the pair distribution function $(\mathrm{PDF})$. This will be developed in later sections but it is important to note that the method determines distances between pairs of atoms; although directional information is lost through the powder averaging of the measurement, the relative atom-atom distances give additional structural detail not easily obtained by other means.

\section{Historical Perspective}

\subsection{The Early Years (1912-1927)}

The observation of rings of 'darkness' on photographic plates placed perpendicular to an X-ray beam incident on a range of non-crystalline materials by Friedrich [2] encouraged Ehrenfest [4] and Debye [3] to simultaneously develop formalisms for scattering from isotropic materials. The Debye equation [3] (and $[5,6])$ gave the scattered X-ray intensity for a monochromatic X-ray beam of wavelength $\lambda$ incident on a collection of electrons as:

$$
I \propto \sum_{n} \sum_{m} \frac{\sin \left[2 k d_{n, m} \sin \left(\frac{\phi}{2}\right)\right]}{\left[2 k d_{n, m} \sin \left(\frac{\phi}{2}\right)\right]},
$$

where $d_{n, m}$ is the distance between the $n^{\text {th }}$ and $m^{\text {th }}$ electron, $\phi$ is the angle between the incident and scattered beam and $k=2 \pi / \lambda{ }^{1}$

\footnotetext{
${ }^{1}$ I have relabelled some of the symbols used by Debye to avoid confusion with those used later in this section.
} 
Whereas Ehrenfest considered the scattering from a simple diatomic gas, Debye's equation was more general, although he advocated initially applying it to measurements of light elements. This was presumably because, although the Debye equation was applicable to all isotropic scatterers, the computational cost meant that at that time it was only viable for the smallest systems. Debye then developed an X-ray camera for these measurements with Scherrer, his student at the time, thus initiating $\mathrm{X}$-ray powder diffraction [7]. Their follow-up paper to this work used a modified DebyeScherrer camera to measure the scattering from liquid benzene [5]. They obtained good data, but the interpretation of the data in terms of the molecular structure using the Debye scattering equation was indeed hampered because the intra- and inter-molecular interference effects overlapped each other.

There then followed a number of experimental X-ray diffraction studies of liquids and, to a lesser extent, gases (see Chapter 9 of [8]), and theoretical attempts to interpret them [9], but it was not until 1927 and a seminal paper by Zernike and Prins that total scattering began to provide more quantitative structural information [10]. Their paper was particularly concerned with understanding how the arrangement of molecules within a liquid, rather than the arrangements of atoms within a molecule, or indeed electrons within an atom, affected the X-ray diffraction pattern. They introduced the idea of a function that represents the probability that a volume element $\mathrm{d} V$ a distance $r$ from the centre of one molecule might contain the centre of another molecule. We would recognise this function as being directly related to the pair distribution function. The crucial insight they had was that when the scattering pattern was formulated in terms of this probability function they could use the properties of the Fourier transform to determine the probability function directly from the X-ray scattering pattern.

The work in $[10]$ therefore forms the basis for total scattering ${ }^{2}$. The integral of their probability function $g(r)$ over a large volume, $V$, is:

$$
\int_{V} g(r) \mathrm{d} v=\rho V+\int_{0}^{\infty} 4 \pi r^{2} g_{0}(r) \mathrm{d} r
$$

where $g_{0}(r)=g(r)-\rho$ and $\rho$ is the number density, the number of atoms (or molecules) per unit volume. This leads to the following expression for the X-ray scattering intensity:

$$
I(\phi)=\frac{1}{2} N A^{2}\left[1+\int_{0}^{\infty} \frac{4 \pi r g_{0}(r)}{s} \sin (s r) \mathrm{d} r\right]
$$

where $\phi$ is the scattering angle, $s=4 \pi \sin \left(\frac{1}{2} \phi\right) / \lambda$ is the momentum transfer, $N$ is the number of scatterers and $A(\phi)$ is the scattering amplitude (the polarisation term was omitted for clarity). If $A^{2}$ is known as a function of $\phi$, then what we now recognise as the total scattering structure factor, $i(s)$, can be calculated:

$$
i(s)=\frac{I(\phi)-\frac{1}{2} N A^{2}}{\frac{1}{2} N A^{2}}
$$

\footnotetext{
${ }^{2}$ Here I have set out these equations from [10] using their notation. The equations described later (Section 3.2) using more 'modern' notation are straightforwardly related to these original formulae.
} 
(a)

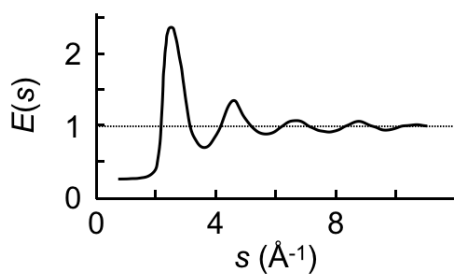

(b)

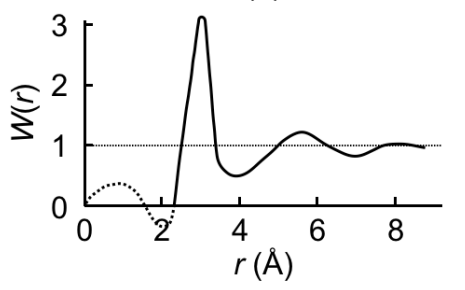

Figure 1. Total scattering from liquid mercury, redrawn and relabelled from [11]. (a) Shows their total scattering structure factor, $E(s)$, which is simply related to $i(s)$ in Equation 5 and (b) their pair distribution function, $W(r)$, similar to $g_{0}(r)$ in Equation 6. (Note that there is a non-standard definition of $s$ in [11]; the $x$-axis in (a) has been rescaled here to be consistent with the more usual definition.)

and

$$
s i(s)=4 \pi \int_{0}^{\infty} r g_{0}(r) \sin (s r) \mathrm{d} r
$$

with the inverse Fourier transform:

$$
r g_{0}(r)=\frac{1}{2 \pi^{2}} \int_{0}^{\infty} \operatorname{si}(s) \sin (r s) \mathrm{d} s
$$

\subsection{Following Zernike and Prins (1930s)}

The first publication of a normalised experimental total scattering structure factor and its Fourier transform was by Debye and Menke in 1930 [11] who measured liquid mercury using a combination of $\mathrm{Cu} \mathrm{K} \alpha$ and Mo $\mathrm{K} \alpha$ radiation to increase the range of momentum transfer $s$ to a $s_{\max }$ of $\sim 11 \AA^{-1}$ whilst retaining good resolution at low-s (see Figure 1). They accounted for experimental factors such as polarisation and absorption, and scaled the scattered intensity data, $I(\phi)$, to follow the total scattering per atom at large $s$ in order to optimise the limiting values of $i(s)$ in Equation 4 . The Fourier transform of this structure factor shows a principle peak just above $3 \AA$ corresponding to the most probable nearest neighbour distance in liquid mercury, in good agreement with much later measurements [12]. A similar measurement and analysis was also made of liquid sodium by Tarasov and Warren in 1936, when they compared the peaks in $g_{0}(r)$ to $\mathrm{Na}-\mathrm{Na}$ distances found in the crystal structure of sodium [13].

It was Warren and co-workers who started applying total scattering methods to crystalline powders. In Warren and Gingrich's [14] 'Fourier integral analysis' reformulation of Zernike and Prins' formulae [10], they make a link to the earlier Debye scattering equation [3], a convenient link for isotropically scattering materials, but one that is not strictly necessary for developing the equations (see Chapter 10 of [15] and Chapter 2 of [16]). ${ }^{3}$ The first total scattering structure factor from a crystalline powder was also given in this article, that of rhombic sulphur [14] (Figure 2a). At the time the structure of this phase was unknown. They used monochromatic Mo K $\alpha$ radiation and a powder diffraction camera with photographic film exposed for 25 hours to produce

${ }^{3}$ Interestingly, there is also a link between total scattering methods and the Patterson function in crystallography. Patterson acknowledges discussions of Warren and Gingrich's paper [14] in his paper [17] which immediately follows [14] in the journal. 
(a)

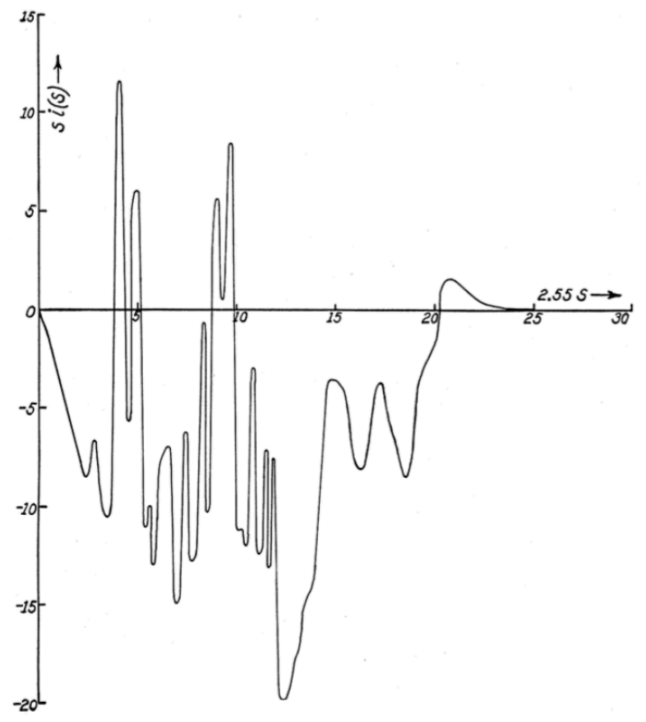

(b)

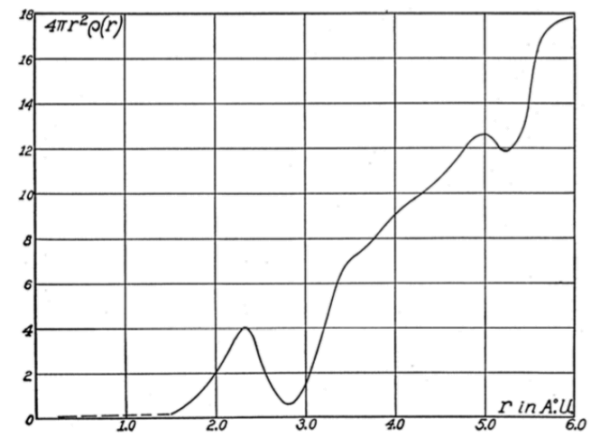

Figure 2. (a) Total scattering structure factor, si(s), and (b) radial distribution function, $4 \pi r^{2} \rho(r),(\rho(r) \equiv$ $g_{0}(r)$ ) from rhombic sulphur (Reprinted figures with permission from [14]. Copyright 1934 by the American Physical Society).

the diffraction pattern. The data were normalised in what we would now recognise as a standard manner to produce a total scattering structure factor over momentum transfers $0.75 \lesssim s \lesssim 9 \AA^{-1}$. The Fourier transform of these data, $\rho(r)$ ( $\equiv g_{0}(r)$ above), has a clear peak centred on $r=2.35 \AA$ with an area corresponding to 2.3 atoms (Figure $2 \mathrm{~b}$ ). This was sufficient to identify that the sulphur atoms form rings or chains with nearest neighbours at this distance, and using this information it was possible to go on to solve the crystal structure. There are a couple of key points about this analysis that these authors appreciated even though the technique was in its infancy. First, no prior knowledge of the crystal structure is needed. The method is 'a perfectly straightforward mechanical operation' for obtaining the relation of each atom to its neighbours. And secondly, the errors introduced in the $i(s)$ during data normalisation will typically only impact the low- $r$ part of $g_{0}(r)$; the form of the physically meaningful part of $g_{0}(r)$ is pretty robust to these errors.

The next paper to highlight was Warren's study of carbon black [18]. The aim of this measurement was to ascertain the nature of the local structure of this form of carbon. The positions and intensities of the first two low- $r$ peaks in $g_{0}(r)$ made it clear that the structure was not diamond-like and that it consisted of graphite-like single layers of carbon atoms, probably in a mix of small graphite crystallites and more random arrangements. This was therefore the first investigation of a substance that was neither crystalline or amorphous; a material that we would probably now call (at least partially) nanocrystalline.

The final four papers in this section use total scattering to compare structures of different crystalline and amorphous phases. The first two papers investigated the structures of the varied phases of phosphorus, the first of which [19] compared the pair distribution functions of 'amorphous' and crystalline black and red phosphorus and showed that they were all very similar with very similar peak positions and areas. The results from crystalline black phosphorus were then used to help solve the crystal structure which, at that time, was unknown. The second paper [20] presented results 
from liquid 'yellow' phosphorus as well as from further measurements on the black and red amorphous forms that revealed an additional lower-s peak in the scattering pattern. This peak was probably observed in [20] and not in [19] because the later work used an X-ray camera with a larger radius. They did however find that including this peak in the Fourier transform made little difference to the resulting pair distribution function at low- $r$, as would be expected. The data were sufficient to show that liquid phosphorus consisted of $\mathrm{P}_{4}$ molecules whereas the solid forms formed networks of 3 -coordinated $\mathrm{P}$ atoms.

The third paper is a study of the structure of silica glass [21], supported by experimental work using monochromatic $\mathrm{Cu} \mathrm{K} \alpha$ and $\mathrm{Mo} \mathrm{K} \alpha$ X-ray radiation described in [22]. The positions and intensities of their pair distribution function of silica nicely showed that the structure was an arrangement of corner-linked $\mathrm{SiO}_{4}$ tetrahedra and, by comparing the diffraction intensity widths of the glass and the crystalline cristobalite phase of $\mathrm{SiO}_{2}$, they also demonstrated that the structures were only similar over a distance of at most $8 \AA$. Their description (expressed in the gendered language prevalent at the time):

'A man sitting on a silicon atom and looking about him would see 4 oxygen nearest neighbors and 4 silicon next nearest neighbors either in cristobalite or the glass; only if he looks farther than the next nearest neighbors could he see any difference'

is a good picture of the similarities and differences between amorphous and crystalline structure networks [21]. However it wasn't until many years later that pair distribution functions from all ambient pressure tetrahedral phases of $\mathrm{SiO}_{2}$ were explicitly used to make a more detailed comparison of the local glass and crystalline structures (see Section 3.4.1). ${ }^{4}$

In the context of the development of the total scattering/pair distribution function method, the measurements made during this period demonstrated a keen understanding of how to optimise the data, such as using more than one incident wavelength to maximise the range of momentum transfers accessible whilst retaining good resolution and the importance of careful data normalisation. The proponents of the method also understood how the pair distribution function could be used to compare the structures of different allotropes irrespective of crystallinity and how it can be an aid to structure solution, especially when samples are only crystalline over a relatively short range. These remain important principles for modern total scattering applications.

\subsection{The Intervening Years (1940 - 1985)}

In this period there were very few groups - indeed perhaps only two [24-27] — using total scattering to study crystalline materials. The section title is perhaps overly negative though, because there were a number of developments of the technique immediately applicable to studies of liquids and amorphous materials that were later applied to crystalline materials. These include the beginnings of the use of neutron diffraction, developments of ideas for multiple measurements such as neutron diffraction from isotopically substituted samples and very early attempts to use computer simulation to model total scattering results. These will be considered in this section.

\footnotetext{
${ }^{4}$ Bernal and Fowler also used comparisons with calculated PDFs based on the various crystalline silica phases
} in their seminal paper on the structure of water, the same paper where they proposed their 'ice-rules' [23]. 


\subsubsection{Franklin's study of carbons}

But before considering these developments to total scattering outside of crystalline systems, it is worth highlighting the first of the three papers referenced in the introductory paragraph above, in which Rosalind Franklin used total scattering to analyse the structure of nanocrystalline carbon in 1950 [24]. This study, part of her wider investigation of coals and other carbons, pre-dated her work on the structure of DNA and was carried out while she was working in Paris [28,29].

Franklin measured X-ray total scattering from a sample of 'Saran', a co-polymer of $\mathrm{C}_{2} \mathrm{H}_{2} \mathrm{Cl}_{2}$ with $\mathrm{C}_{2} \mathrm{H}_{3} \mathrm{Cl}$, which had been heated to $1000^{\circ} \mathrm{C}$ in nitrogen to obtain pure carbon. The fired sample was ground and mixed with a small amount of glue to form a thin plate and X-ray photographs were taken using monochromatic $\mathrm{Cu} \mathrm{K} \alpha$ and Mo K $\alpha$ X-rays. Broad diffraction peaks associated with ( $h k 0)$ Bragg reflections from graphite, together with a weak shoulder to the significant small angle scattering and corresponding to the (002) reflection, were obtained (Figure 3a). These data, when Fourier transformed to produce a pair distribution function (Figure 3b), were used to show that the material's structure consisted of $65 \%$ 'highly perfect and planar graphite-like layers of diameter $16 \pm 1 \AA^{\prime}$ and $35 \%$ that was highly disordered. Approximately $55 \%$ of the layers were arranged in pairs. This study is an excellent example of the issues involved when trying to correct and analyse total scattering data. These include: the difficulties in making the subtraction in Eqtn (4) and its subsequent impact on unphysical low- $r$ features in the resultant pair distribution function; how lack of sufficiently high- $s$ data causes truncation ripples in the PDF (through Eqtn (6)); and ways that this behaviour might be mitigated using appropriate smoothly varying modification functions. The paper also contains the first example of how a PDF can be constructed from a model collection of atoms and compared with the data in real and reciprocal space. A calculation based on a continuous single sheet of graphite is shown as a dotted line in Figure 3b; subsequent variation of this PDF's scale and high- $r$ damping to obtain better agreement with the data provided an estimate of the amount of crystalline material and size of crystalline regions, respectively. Furthermore, because Franklin discusses all these issues in such careful detail, the paper provides an excellent pedagogical reference for the total scattering method at the time.

\subsubsection{Structural studies of metal powders and selenium-based glasses}

The second and larger body of work [25-27] mentioned in the introduction to this period was associated with a group from Massachusetts Institute of Technology who were honing X-ray PDF methods so that they could use them to investigate the structure of amorphous selenium and arsenic-selenium. The significance of their work for this review is twofold: they used PDF data from crystalline phases in their analysis of their glass structures in a more rigorous manner than had been done previously; and they developed Monte Carlo methods to produce model structures from fits to their PDF data. Both might reasonably be considered forerunners of later analysis methods which came to be widely used on PDF data from crystalline materials.

In their first paper, they measured the PDF from lead powder close to its melting point [25]. This sample was carefully chosen, partly so that they might investigate the melting phenomenon, but also because the high degree of disorder suppressed the Bragg peak intensities at high momentum transfers, $k$, making the Fourier transform to the PDF more straightforward despite the limited range of $k=4 \pi \sin \theta / \lambda$ available from Mo $\mathrm{K} \alpha \mathrm{X}$-ray radiation. ( $k$ was increasingly replacing $s$ as the label for momentum transfer at this time; we would now typically label it $Q$.$) Their PDF data$ 

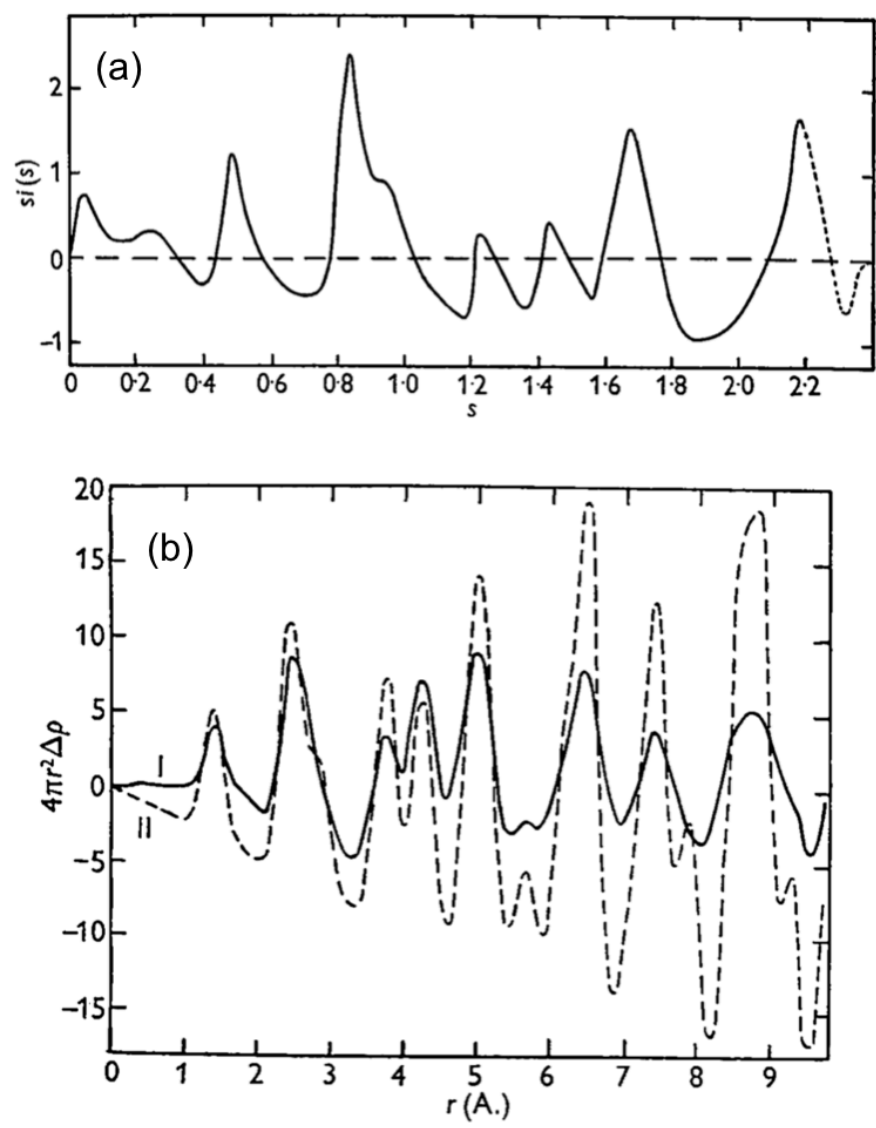

Figure 3. (a) Total scattering structure factor, si(s), and (b) pair distribution function, $4 \pi r^{2} \Delta \rho(r)$, of carbon. (b) shows the experimental data (full line I) and a calculation based on an infinite graphitic layer (dashed line II). In these figures $s=2 \sin \theta / \lambda$, i.e. the more conventional definition of $s$ divided by $2 \pi$, and $\Delta \rho \sim D(r)$ (see Equation 13). (Reproduced from [24] with permission of the International Union of Crystallography.) 


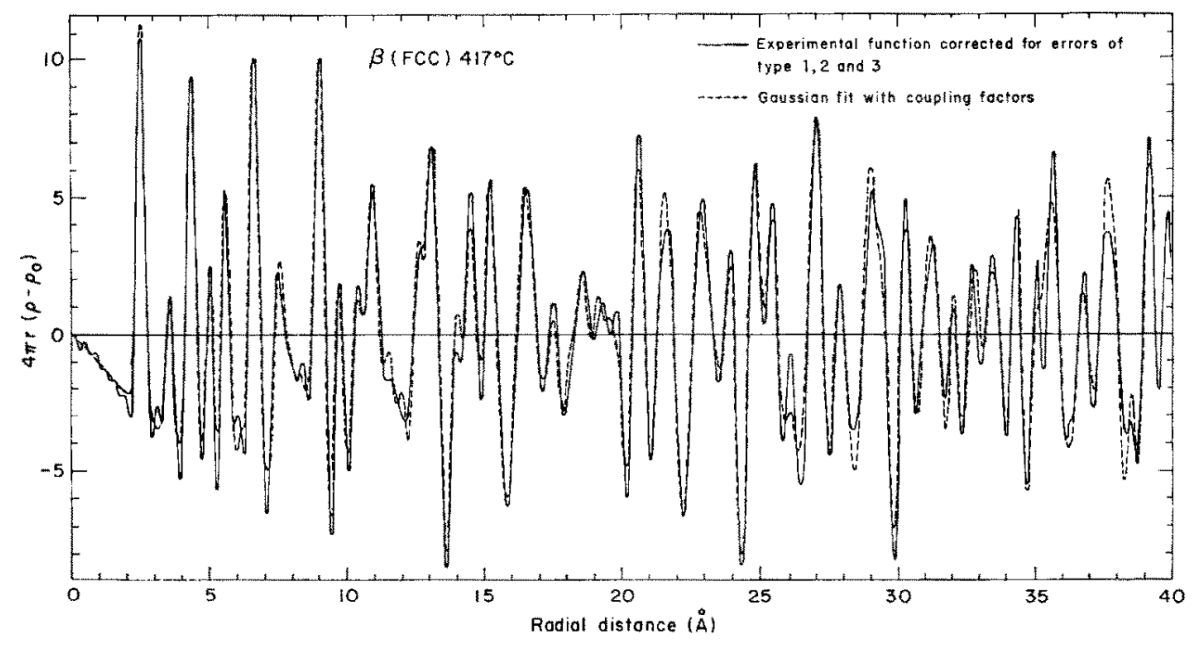

Figure 4. Pair distribution function, $4 \pi r\left(\rho(r)-\rho_{0}\right)\left[=4 \pi r g_{0}(r)\right]$, of cobalt at $417^{\circ} \mathrm{C}$ and in its fcc-structured phase. Solid line is the fully corrected experimental data and the dashed line is a fit based on Gaussian functions placed at $r$-values defined by $\mathrm{Co}-\mathrm{Co}$ distances in the crystal structure and broadened by experimental factors and terms to account for thermal vibrations, see main text and [27] for details. (Reprinted from [27], with permission. Copyright 1967 from Elsevier.

were analysed in terms of Gaussian-broadened peaks whose positions were obtained from atom pair distances in the average fcc lattice and whose widths varied depending on the degree of coupling between the atoms' thermal vibrations. An excellent agreement to the experimental PDF was obtained, showing that the Gaussian broadening increased as the radial distance increased (and as coupling between their thermal vibrations decreased). It is notable that this methodology was much later developed into refinement methods collectively termed 'small-box' methods (see Section 3.4.2).

Two of their later papers further developed the analysis methods from [25] in investigations of two crystalline phases of cobalt [27] and the crystalline and molten phases of aluminium $[26] .{ }^{5}$ In these examples the thermal disorder was much reduced compared to that of their lead sample and this necessitated a more careful treatment of sources of error such as truncation and the effect of $Q$-space resolution. They achieved a very good fit to the data from the high-temperature $\beta$-phase of cobalt (see Figure 4) and obtained lattice parameters, vibrational amplitudes and Debye temperatures from both phases from the PDF data.

Their next work was a study of the structure of glassy selenium [31]. Following the methods developed in [25] they obtained vibration coupling coefficients by fitting Gaussian peaks to the measured PDF from hexagonal selenium. This PDF was then compared to that of amorphous selenium, showing that some agreement might be obtained if the PDF from the refined hexagonal phase was modified by assuming a larger independent mean-squared displacement and a $13 \AA$ microcrystallite size. Better agreement was possible by also expanding the hexagonal structure to account for the differences in density, but the best was obtained with the PDFs calculated from monoclinic phases of selenium. As an alternative to using broadened Gaussian peaks fixed to atom-atom distances in the average crystalline structure, the authors also constructed spherical configurations of around 100 atoms based on the crystal structures and ran-

\footnotetext{
${ }^{5}$ Similar measurements were also being carried out around the same time on aluminium just above and below $T_{\mathrm{M}}$ in Germany [30]. The Al powder PDF data were used to validate calculations of the liquid structure based on the crystal structure.
} 
domly moved atoms one at a time until the PDF calculated from the configuration was in good agreement with the experimental data. Although they only accepted moves that improved the agreement, and their configurations were too small to consider using periodic boundary conditions, it is clear that this is in some sense a forerunner of the reverse Monte Carlo 'big-box' methods developed much later (see Section 3.4.1). Significantly - based on their experimental work and careful analysis - they were able to suggest that the glass structure did predominantly consist of $\mathrm{Se}_{8}$ rings. Their later papers $[32,33]$ developed these modelling methods further with more advanced Monte Carlo algorithms and applying them to binary arsenic-selenium glasses.

\subsubsection{Neutron total scattering from liquids and glasses}

Neutron diffraction from powders was established through the experimental work at an Oak Ridge reactor pile in the United States [34,35]. This was followed by measurements of liquid structure factors using a diffractometer at an Argonne reactor that was modified to increase count-rate [36]. This paper from 1950 gives an 'apparent' differential cross-section of powdered graphite, albeit over a limited range of momentum transfer and only measured to establish the instrumental resolution, but nonetheless this is probably the first publication of a measured neutron total scattering structure factor from a crystalline powder. Furakawa [37] provides an excellent summary of this early neutron and X-ray liquid diffraction work.

A significant advance was made in determining liquid structure with the development of the technique of neutron diffraction with isotopic substitution [38]. In this, neutron diffraction from chemically identical samples are measured, with each sample isotopically enriched by different amounts. Provided the different isotopes have sufficiently distinct neutron scattering lengths, the total scattering structure factors from these data - which are scattering length weighted sums of partial structure factors (see Equation 8 in Section 3.2) — can be combined to separate out contributions from different pairs of atom types. Although isotopic substitution total scattering methods have not been used for crystalline systems, related combinatorial methods to directly obtain partial PDFs from X-ray and neutron total scattering data, as applied to amorphous $\mathrm{GeO}_{2}$ [39] for example, led to the simultaneous fitting of neutron and X-ray total scattering within 'big-box' refinements as a way to further constrain the resultant atomistic models (see Section 3.4.1). Indeed many of these 'big-box' computational methods were also initiated within the liquid structure community, as was a strong focus on experimental precision and accuracy [40].

A few people working on liquid structures using diffraction data realised that for some problems the mathematical inversion of isotopic substitution total scattering data was ill-conditioned, especially if the data were of lower than optimal quality or if the isotope neutron scattering length contrast was low (e.g. [41]) They suggested that the structural descriptions might be improved if the datasets were instead used to refine a three-dimensional atomistic model of the structure, since then the resulting partial PDFs would at least be consistent with a physical collection of space-filling atoms. Furthermore, the atomistic models could also be interrogated to provide structural information such as bond-angle distributions and other longer-ranged correlations that were impossible to extract from a mathematical treatment of isotopic substitution neutron data. These ideas were incorporated into programs such as the Empirical Potential Structure Refinement (EPSR) program [42,43] and Dissolve [44]. 


\section{The PDF in Crystallography Today}

\subsection{Modern Developments}

The first PDF measurements from a crystalline sample within the 'modern' era were used to study the local structure of recently discovered quasicrystals in the late 1980's, initially with measurements using anomalous X-ray diffraction $[45,46]$ and then using neutron diffraction [47], taking advantage of the new developments of synchrotron Xray and spallation neutron sources, respectively. Around the same time we were using the newly developed reverse Monte Carlo modelling method [48,49] to analyse total scattering from crystalline superionic conductors as they approached their melting temperatures [50]. This work led to the program RMCProfile [51], a method for refining 'big-box' supercell models to a range of experimental data including total scattering and the Bragg profile. Takeshi Egami's group were also using PDFs to investigate the local structure of high-temperature superconductors and other topical metal oxide materials [52-54] and these led to the development of the PDFFIT/PDFgui program [55] for refining 'small-box' models through fitting PDF data.

It is the developments of these four techniques - measurements at spallation neutron sources and X-ray synchrotrons, 'big-box' and 'small-box' analysis methods - which are the principle reasons for the widespread use of PDF methods in crystallography today. This section will describe all four in some detail, after first outlining the modern total scattering formalism which will be referred to in the remaining sections of this review.

\subsection{Formalism}

Since PDFs have been used in several distinct fields, the formalism is often different depending on the scientific 'pedigree' of those writing about the topic. In this review we will follow the work of [56]. ${ }^{6}$ For neutrons, the total scattering structure factor, $F(Q)$, is the interference term of the differential neutron scattering cross-section, $\frac{1}{M} \frac{\mathrm{d} \sigma}{\mathrm{d} \Omega}$, i.e.

$$
F^{N}(Q)=\frac{1}{M} \frac{\mathrm{d} \sigma}{\mathrm{d} \Omega}-\sum_{i=1}^{n} c_{i} \overline{b_{i}^{2}}
$$

where there are $M$ atoms and $n$ atom species in the material. $4 \pi \sum_{i} c_{i} \overline{b_{i}^{2}}$ is the neutron total scattering cross-section of the material. The superscript $N$, which is usually omitted in the literature, is used here to distinguish between these neutron-specific equations and those for X-ray total scattering below. Alternatively

$$
F^{N}(Q)=\sum_{i, j=1}^{n} c_{i} c_{j} \bar{b}_{i} \bar{b}_{j}\left[A_{i j}(Q)-1\right]
$$

a weighted sum of $A_{i j}(Q)$, the partial structure factors. $c_{i}$ and $\bar{b}_{i}$ are the proportion and neutron coherent scattering length of atom $i$, respectively. $A_{i j}(Q)$ are related to the partial PDFs, $g_{i j}(r)$, by the sine Fourier relationships

$$
A_{i j}(Q)-1=\rho_{0} \int_{0}^{\infty} 4 \pi r^{2}\left[g_{i j}(r)-1\right] \frac{\sin Q r}{Q r} \mathrm{~d} r
$$

\footnotetext{
${ }^{6}$ Please refer to [56] for the relationships between the equations written here and other commonly used formalisms.
} 
and

$$
g_{i j}(r)-1=\frac{1}{(2 \pi)^{3} \rho_{0}} \int_{0}^{\infty} 4 \pi Q^{2}\left[A_{i j}(Q)-1\right] \frac{\sin Q r}{Q r} \mathrm{~d} Q,
$$

with the number density, $\rho_{0}$, typically given in atoms per $\AA^{3}$. As Equations 9 and 10 include no experimental terms apart from the sample density, they are applicable to neutron and X-ray total scattering.

The neutron total radial distribution function, or PDF, is defined as (cf. Equation 8)

$$
G^{N}(r)=\sum_{i, j=1}^{n} c_{i} c_{j} \bar{b}_{i} \bar{b}_{j}\left[g_{i j}(r)-1\right]
$$

such that $F^{N}(Q)$ and $G^{N}(r)$ are related to each other via a sine Fourier transform in an identical manner to that of $A_{i j}(Q)-1$ and $g_{i j}(r)-1 . g_{i j}(r)$ can be calculated straightforwardly from a collection of atoms

$$
g_{i j}(r)=\frac{n_{i j}(r)}{4 \pi r^{2} \mathrm{~d} r \rho_{j}}
$$

where $n_{i j}(r)$ are the number of atoms of type $j$ between distances $r$ and $r+\mathrm{d} r$ from an atom of type $i$ and $\rho_{j}=c_{j} \rho_{0}$, although the volume element $4 \pi r^{2} \mathrm{~d} r$ might need to be modified for cases where the collection of atoms is smaller than the range of $r$ being evaluated (e.g. when there isn't a full spherical shell of atoms of radius $r$ around all atoms in the model such as might be the case for a nanoparticle). A common alternative normalisation for the $\mathrm{PDF}$ is $D(r)$

$$
D^{N}(r)=4 \pi r \rho_{0} G^{N}(r)=\left(\sum_{i=1}^{n} c_{i} \bar{b}_{i}\right)^{2} G^{\mathrm{PDF}}(r),
$$

which, by scaling by $r$, emphasises the high- $r$ features of the PDF more prominently than the low- $r$ features. $G^{\mathrm{PDF}}(r)$ is the normalisation used in the PDFgui program described in Section 3.4.2.

The equivalent $\mathrm{X}$-ray formalism is a little more complex because the neutron scattering lengths, $\bar{b}_{i}$, used in the equations above must be replaced by $Q$-dependent X-ray scattering factors, $f_{i}(Q)$. Equation 7 then becomes

$$
F^{X}(Q)=\left[\frac{1}{N} \frac{\mathrm{d} \sigma}{\mathrm{d} \Omega}-\sum_{i=1}^{n} c_{i} f_{i}(Q)^{2}\right] /\left(\sum_{i=1}^{n} c_{i} f_{i}(Q)\right)^{2} .
$$

$\left(\sum_{i=1}^{n} c_{i} f_{i}(Q)\right)^{2}$ is a so-called 'sharpening' term used to enhance the high- $Q$ scattering by counteracting the natural fall-off in intensity due to the form of the X-ray scattering factors. Often $\sum_{i=1}^{n} c_{i} f_{i}(Q)^{2}$ is used as an alternative sharpening term (c.f. Equation 4); see [57] for a discussion of their relative merits. Following Equation 8

$$
F^{X}(Q)=\sum_{i, j=1}^{n} c_{i} c_{j} f_{i j}(Q)\left[A_{i j}(Q)-1\right]
$$


with

$$
f_{i j}(Q)=f_{i}(Q) f_{j}(Q) /\left(\sum_{i=1}^{n} c_{i} f_{i}(Q)\right)^{2} .
$$

Although the sine Fourier transform of $F^{X}(Q)$ is typically calculated thus

$$
G^{X}(r)=\frac{1}{(2 \pi)^{3} \rho_{0}} \int_{0}^{\infty} 4 \pi Q^{2} F^{X}(Q) \frac{\sin Q r}{Q r} \mathrm{~d} Q,
$$

it is important to realise that

$$
G^{X}(r) \neq \sum_{i, j=1}^{n} c_{i} c_{j} j_{i j}(r)\left[g_{i j}(r)-1\right]
$$

where $j_{i j}(r)$ would be the Fourier transform of $f_{i j}(Q)$; the two $r$-dependent terms on the right hand side should be convoluted in order to form $G^{X}(r)$. To simplify the calculation of $G^{X}(r)$ from the partial PDFs, it is usually assumed that the $Q$ dependency of $f_{i}(Q)$ is the same for all atoms, each scaled by the effective number of electrons for that atom, i.e. $f_{i}(Q)=K_{i} f_{e}(Q)$. Here $K_{i} \approx Z_{i}$ for neutral atoms and

$$
f_{e}(Q)=\sum_{i=1}^{n} c_{i} f_{i}(Q) / \sum_{i=1}^{n} c_{i} Z_{i}
$$

is an average scattering factor per electron for the material. It depends on the material as to how good an approximation this is, but typically it becomes less good for materials containing atoms with very different numbers of electrons. ${ }^{7}$ Combining the definition of $f_{e}(Q)$ in Equation 19 with the effect of the sharpening term in Equation 16 removes the $Q$-dependency of $f_{i j}(Q)$ and

$$
G^{X}(r)=\sum_{i, j=1}^{n} c_{i} c_{j} \frac{K_{i} K_{j}}{\left(\sum_{i=1}^{n} c_{i} Z_{i}\right)^{2}}\left[g_{i j}(r)-1\right],
$$

a simple weighted sum of the partial PDFs, as in the neutron total scattering case (Equation 11). Note that this simplification is dependent on using $\left(\sum_{i=1}^{n} c_{i} f_{i}(Q)\right)^{2}$ as the sharpening function. Should this approximation be deemed inapplicable then it is still possible to compare models to the X-ray PDF: the partial PDFs, $g_{i j}(r)$, are individually transformed to partial structure factors, $A_{i j}(Q)$, via Equation 9; these are summed together to form the total scattering structure factor, $F^{X}(Q)$, (Equation 15); and this is then Fourier transformed back to the total PDF, $G^{X}(r)$, through Equation 17.

\footnotetext{
${ }^{7}$ The applicability, or otherwise, of this approximation for analysing total scattering using silica as an example is discussed in detail in pages $132 \mathrm{ff}$ and Figure 10.8 of [15]; also see the impact of this approximation when calculating the O-D contribution to the X-ray PDF of hydrogrossular in Section 3.6.3.
} 


\subsubsection{Impact of limited total scattering data}

Of course when applying these equations in practice further limitations come in to play. The most significant of these arises from the limited range of $Q$ available in a measurement where the sine Fourier transform to produce the PDF which ideally integrates between 0 and $\infty$ (e.g. Eqtn 10), is replaced experimentally by a summation between $Q_{\min }$ and $Q_{\max }$. The missing parts of a measured $F(Q)$ at low- and high$Q$ mean that an experimental PDF will lack low- and high-frequency components, respectively. The broad oscillations in the $\mathrm{PDF}$ originating from low- $Q$ data only become significant if there are density fluctuations in the material that would give rise to 'small angle' scattering and can be ignored for most bulk crystalline materials. However, the lack of high-frequency features in the PDF cause the PDF peaks to broaden with Fourier ripples that are more problematic, especially if $Q_{\max }$ is relatively small or there are very well-defined bonds in the material (such as $\mathrm{C} \equiv \mathrm{N}$ bonds). The way that the instrumental resolution broadens the Bragg peaks will also impact on the PDF; the peak broadening causes features at higher- $r$ to be suppressed. And imprecise data normalisation and finite measuring times will introduce systematic and statistical errors, respectively. However, the precise form of these experimental modifications are beyond the scope of this review and have been considered thoroughly elsewhere (see for example [58-60]).

\subsection{PDF Instrumentation}

\subsubsection{Neutron total scattering diffractometers}

As alluded to in Section 2.3.3, neutron diffraction became the method of choice for measuring total scattering from liquids and amorphous materials. This was because there is no fall off in intensity at high- $Q$ from the effect of $Q$-dependent scattering factors (c.f. Equations 7 and 14), 'hot' sources at reactors provide monochromatic neutron beams with (relatively) short wavelengths, and through careful measurement and data normalisation (see later in this section) it is possible to place the total scattering on an absolute scale. These reactor-based instruments, such as D4c [61] at the Institut Laue-Langevin in France, have been used for PDF measurements from powdered crystalline samples, but they are not ideal because the maximum $Q$ available is still (relatively) low and the high-fluxes needed for the measurements are obtained through a compromised (i.e. broadened) instrumental resolution. A $Q_{\max } \simeq 23 \AA^{-1}$ and $Q$-resolution of around 2.25\% [62] on D4c is sufficient for the majority of liquid and glass diffraction patterns, but it can be limiting for highly crystalline materials. The $Q$ resolution broadens Bragg peaks, giving rise to less well resolved Rietveld refinements, and suppresses the intensities of high- $r$ features in the PDF. A small $Q_{\max }$ means that $F^{N}(Q)$ is unlikely to have smoothly approached its limiting value at $Q_{\max }$ and the PDF obtained from the sine Fourier transform of $F^{N}(Q)$ will include noise associated with this, albeit somewhat ameliorated by the associated broad $Q$-resolution at $Q_{\max }$.

The majority of neutron PDF measurements from crystalline materials are now carried out on diffractometers at time-of-flight spallation sources. There are several such instruments world-wide that are ideally suited to PDF measurements, including the GEM [63,64] and Polaris [65] diffractometers at the ISIS Facility in the UK, and PDF measurements make up a substantial proportion of the experimental program on these two general purpose powder diffractometers. A spallation neutron source produces pulses of neutrons with a spectrum of wavelengths that are detected using 
position sensitive detectors, which also record the time the neutrons take to arrive at the detector from the neutron source via the sample. Time-of-flight neutron powder diffractometers have large banks of fixed detectors covering a wide range of scattering angles and, for PDF measurements, typically record data for wavelengths in the range $0.1<\lambda<4.0 \AA .{ }^{8}$ The combination of a broad range of wavelengths, including very short wavelengths, and a substantial detector coverage of scattering angles means that a measurement will collect data over a very wide range of momentum transfers, typically $0.1<Q<50 \AA^{-1}$. Furthermore, the characteristics of the source can be tailored to provide good $Q$-resolution in the back-scattering detectors, e.g. $\Delta Q / Q=$ $0.3 \%$ for Polaris [65].

A PDF measurement on a neutron time-of-flight instrument is qualitatively the same as a standard powder diffraction measurement, except that the data are collected for longer to improve counting statistics, especially for the diffuse scattering at high- $Q$, and additional measurements are required to characterise backgrounds fully and to enable absolute data normalisation. A cylindrical sample is measured with separate measurements for the empty cylindrical sample container, empty sample environment (furnace, cryostat etc.), empty instrument and a V-Nb rod of similar dimensions to the sample. Vanadium has a very small negative neutron scattering length and hence a very small coherent neutron scattering cross-section. This is balanced by alloying with 5.14 atomic \% niobium which doesn't perturb the vanadium structure significantly and, by having a positive neutron scattering length, results in a calibration rod with an overall neutron scattering length of zero. The scattering from a V-Nb rod is therefore almost completely incoherent and is used as a measure of the incident neutron flux as measured by each instrument detector. Programs exist, such as GudrunN $[66]^{9}$, that take all these different data-sets and use them to accurately account for all experimental 'artefacts' such as backgrounds, attenuation, multiple scattering, Placzek corrections etc. and to merge the data from different detector banks to produce a single absolutely normalised neutron total scattering structure factor.

Absolute normalisation is important, not just as a check of data quality, but also for comparison with other data-sets and so that simple parameters such as co-ordination numbers might be calculated. For most dense samples that don't scatter neutrons at low- $Q$ (from density fluctuations etc.) the limiting values of $F^{N}(Q)$ are

$$
\begin{aligned}
F^{N}(Q \rightarrow 0) & \approx-\sum_{i=1}^{n} c_{i} \bar{b}_{i}^{2} \\
F^{N}(Q \rightarrow \infty) & =0
\end{aligned}
$$

and the limiting values for the corresponding PDF are

$$
\begin{aligned}
G^{N}\left(r<r^{0}\right) & =-\left(\sum_{i=1}^{n} c_{i} \bar{b}_{i}\right)^{2} \\
G^{N}(r \rightarrow \infty) & =0,
\end{aligned}
$$

\footnotetext{
${ }^{8}$ Although in a neutron diffraction measurement, shorter neutron wavelengths integrate over a greater range of energy transfers within the sample and at closer to constant $Q$, and are therefore preferred for total scattering (see introductory chapters of the GudrunN program manual, available from [67])

${ }^{9}$ The best way to find out more about this program and its graphical user interface, GudrunGUI, and indeed further descriptions of the data corrections listed here, is to download the manual provided with the program, available from [67].
} 
where $r^{0}$ is the shortest distance that any pair of atoms in the material might approach each other (see [56] for further details, including how co-ordination numbers might be obtained from $G^{N}(r)$ ).

\subsubsection{X-ray total scattering diffractometers}

Beamline X13-A of the National Synchrotron Light Source (NSLS) of Brookhaven National Laboratory was used for the anomalous diffraction studies of quasicrystals [45,46] mentioned earlier (Section 3.1). Shortly afterwards, Station 9.1 [68] of the Daresbury Synchrotron Radiation Source (SRS) became the key diffractometer for establishing synchrotron X-ray PDF studies in the U.K. Although this work mainly revolved around measurements of liquid and amorphous samples, a number of important measurement principles were trialled using this diffractometer. This flexible high-resolution, high-intensity powder diffractometer viewed a three-pole five Tesla superconducting wiggler with a critical wavelength of around $1 \AA$. Various detection systems were available and it was possible to tune the incident wavelength precisely and with good stability. Shorter wavelengths were available for total scattering measurements; $0.5 \AA$ was typical (e.g. as used for a study from that period of the structure of a rare-earth metaphosphate glass [69]). Methods were tested to reduce the Compton scattering, thought to be a hindrance in data normalisation at high- $Q$ where the Compton scattering dominates. One such method was the Warren-Mavel technique [70], whereby the X-rays scattered from the sample shine on a foil chosen to have an absorption edge just below the energy of the incident X-ray beam. The detector records the fluorescence from the foil and only the elastically scattered X-rays cause the foil to fluoresce; the lower energy incoherently scattered X-rays (i.e. Compton) do not. This was used with some success on Station 9.1 [71] at the $\mathrm{Ag} K$-edge, and in a study of silver-ion conducting glasses at the Zr $K$-edge [72].

In the 1990's there was a debate about the best way to collect total scattering data. Some thought that it was important to remove extraneous scattering that impacted on the ability to correct the scattering data experimentally, advocating methods such as the Warren-Mavel technique described in the previous paragraph. This view had implications on the types of detection systems that could be used and any treatment of the scattered beam (fluorescence detection or further monochromatisation [73], for example) reduced the overall count-rate. It also precluded the use of area detection systems that were being developed at the time. Others advocated the use of 'black' detectors, i.e. detectors that recorded all X-rays scattered from the sample. They argued that the way that the inelastic Compton scattering signal varied in peak intensity, width and position, $\Delta E$, relative to the elastic signal as a function of scattering angle made a complete removal virtually impossible and that a subsequent subtraction of a partially — or even almost completely — removed signal (e.g. by incorporating a Ruland monochromator cut-off function in the analysis [74,75]) was more difficult than removing the larger, but total, Compton contribution which is well described theoretically. Furthermore, they believed that the greater statistical accuracy at high- $Q$ possible with 'black' detectors outweighed the poorer signal to background, especially since area detectors could then be used to further increase count-rate. Area detectors were trialled on Station 9.1, initially using flat image plates [76], before installing a curved device [77].

Daresbury and NSLS were so-called 'second-generation' synchrotron sources. It was the advent of 'third-generation' sources that really saw PDF methods using X-rays firmly established. Third-generation sources, such as the ESRF in France, the Ad- 
vanced Photon Source in the USA, Diamond Light Source in the UK etc. have far higher brightness and typically operate at higher energies than the synchrotrons they replaced, thus providing higher fluxes at shorter wavelengths. Shorter wavelengths give access to higher momentum transfers, and the higher flux at short wavelengths combined with large 2D detectors enabled very quick PDF data collections. An early example using a flat-plate sample of $\mathrm{Ga}_{1-x} \operatorname{In}_{x}$ As and a scanning single detector achieved a $\mathrm{Q}_{\max }=45 \AA^{-1}$ with an incident X-ray energy of $60 \mathrm{keV}\left(\lambda=0.206 \AA^{-1}\right)$ at the Cornell High Energy Synchrotron Source $[78,79]$. However the vast majority of diffractometers being used for PDF measurements do not achieve such a high $Q_{\max }$ because, although they might use still shorter X-ray wavelengths, their detectors do not collect data to such high scattering angles. (The measurement on $\mathrm{Ga}_{1-x} \mathrm{In}_{x}$ As would have collected data to $2 \theta \sim 110^{\circ}$ [79].) Most PDF diffractometers use a flat square pixellated detector placed perpendicular to the incident beam, often such that the straight though beam would pass through the centre of the detector. This significantly limits the maximum scattering angles available even if the detector is placed very close to the sample. Furthermore it is much harder to normalise data from area detectors because they are inherently more 'noisy' and harder to shield from sources of background. So even if it is possible to measure to $Q \sim 40+\AA^{-1}$, through use of very high energy X-rays for example, it is often not possible to correct the data reliably at the higher $Q$-values and $Q_{\max }$ of $25-35 \AA^{-1}$ is more typical.

Although many powder diffractometers at synchrotrons are used for PDF measurements ${ }^{10}$, there are currently only two diffractometers that were designed from the outset for PDF measurements from crystalline samples, XPDF (I15-1) at Diamond [82] and 11-ID-B at the APS [83]. Both of these use fixed incident wavelengths and simple beam line optics to deliver a stable, intense, clean beam onto the sample. Area detectors are used to collect the scattered radiation, placed behind the sample and perpendicular to the beam. On XPDF the 'PDF' detector is arranged so that the transmitted beam would hit the bottom corner of the detector, whereas on 11ID-B the detector is placed symmetrically about the path of the transmitted beam. The former arrangement accesses the highest range of $Q$ for a given incident wavelength and sample-to-detector distance whereas the latter-by measuring the whole Debye-Scherrer cone simultaneously - maximises count-rate. This arrangement is particularly effective for rapid measurements and 11-ID-B is often used for in-situ PDF measurements $[84,85]$. This so-called "Rapid Acquisition PDF" or RA-PDF is especially effective, for example, for following the growth of nanoparticles out of solution [86].

The treatment of the data to produce a PDF is fairly routine, with many of the principles determined many years ago (e.g. [24] amongst others). Most experiments, as with neutron total scattering, collect data in capillary (cylindrical) geometry, although in contrast to the neutron case it is more usual for the beam to be smaller than the internal diameter of the capillary. With these small samples and small beam sizes the powders should be finely ground and, where possible, the capillary is rotated to improve powder averaging. Measurements are also made of an empty capillary (or other appropriate sample container) and instrument background. For area detectors the first stage is to azimuthally average the $2 \mathrm{D}$ detector images to produce a $1 \mathrm{D}$ pattern as a function of scattering angle, taking into account slight misalignments of the detector and sample, changes in effective detector pixel thickness from parallax,

${ }^{10}$ Such as high-resolution instruments with detectors behind analyser crystals (e.g. ID22 - previously ID31[80]at the ESRF) or those using microstrip detectors that cover a wide $2 \theta$ range (e.g. MS-X04SA at the SLS, PSI [81]). 
intensity variations due to X-ray polarisation, as well as detector pixel responses and dark count background etc. [87].

The experimental 'artefact-free' one-dimensional scattering pattern is then normalised to account for backgrounds, absorption, multiple scattering and Compton scattering and placed on an absolute scale using programs such as GudrunX [57]. Here the absolute scaling is a little more arbitrary than in the neutron total scattering case because there isn't an X-ray equivalent to a vanadium scatterer. Instead the data are scaled such that they follow the sum of the theoretical independent coherent (i.e. $\left.\sum_{i=1}^{n} c_{i} f_{i}(Q)^{2}\right)$ and incoherent (Compton) scattering functions. This is usually achieved using an iterative method; see the GudrunX manual [67]. Another program PDFgetX3 [139] follows a more $a d-h o c$ approach to the data normalisation by fitting a smoothly varying $Q$-dependent additive term to the structure factor to account for the errors in the measured data. This has the benefit of being very straightforward to use and computationally fast, but does not provide an absolute normalisation or necessarily produce 'well-behaved' structure factors. This is often most evident at low- $Q$, probably because the program does not subtract the background data in as rigorous a manner as GudrunX. It relies on the observation that the low- $r$ part of the PDF - the part typically used in subsequent analysis - is relatively insensitive to smoothly-varying and unphysical errors in the low- $Q$ part of the structure factor and, provided that further analysis only requires an arbitrarily-scaled PDF, reasonably corrected data are produced.

As a postscript to this section on X-ray total scattering diffractometers, it is notable that there has been a recent return to laboratory-based diffractometers for PDF measurements. A handful of diffractometer manufacturers have developed instruments based on silver $\mathrm{K} \alpha \mathrm{X}$-ray sources, some coupled with focusing mirrors to remove the Bremsstrahlung radiation, and small efficient detectors to measure PDFs with a $Q_{\max } \simeq 20 \AA$. The count-rate is low and the resolution means that they are more likely to be used for liquids and amorphous samples or crystalline samples with simple structures or low crystallinity, but well-normalised data are obtained using programs such as GudrunX, which was developed with these diffractometers in mind [57]. Other university researchers are pairing monochromatic silver sources with area detectors with some success [89], providing higher-resolution data with collection times of the order of hours rather than the one or two days from the commercial instruments.

\subsection{PDF Data Analysis and Modelling}

\subsection{1. 'Big box' modelling and RMCProfile}

The reverse Monte Carlo (RMC) modelling method [48,49] was developed in the late 1980 's as a way to create atomistic models of liquids and amorphous materials from diffraction data which, given the samples under study, necessarily meant that total scattering data were used. The early liquid and amorphous starting models were simply constructed by randomly filling a box with atoms to give the correct chemical composition and density. This approach was quite inefficient and starting non-crystalline models are now more likely to have been created by other means such as molecular dynamics simulation, with the RMC method used to refine the models against the total scattering data (e.g. [90]).

The principle of RMC is very straightforward. The atoms within this large box of atoms - which is additionally a supercell of the unit cell if the sample is crystallineare moved randomly one at a time a random amount and after each move relevant 
functions are calculated from the atoms. The differences between these calculated functions and the equivalent experimental ones are then used to calculate a $\chi^{2}$-like cost function; if $\chi^{2}$ decreases after the move the move is accepted and if it increases it is accepted with a certain probability related to the size of the change in $\chi^{2}$. The process is repeated many times until there is no improvement in $\chi^{2}$ with additional atom moves and the atomistic model is said to have converged. The algorithm is therefore very similar to the Monte Carlo method [91] except that the change in $\chi^{2}$ is used to drive the simulation instead of the change in energy.

As mentioned in section 3.1, the first use of RMC on a crystalline material was to investigate the structural disorder as $\mathrm{AgBr}$ approached melting [50]. Rocksalt-structured $\mathrm{AgBr}$ shows increasing ionic conductivity as the melting point at $T_{\mathrm{M}}=701 \mathrm{~K}$ is approached, reaching values of $\simeq 1 \Omega^{-1} \mathrm{~cm}^{-1}$ just below $T_{\mathrm{M}}$, and thought to be due to increasing concentrations of cation Frenkel interstitial defects. Neutron total scattering was measured on the D20 diffractometer at the ILL at a number of temperatures to within $2 \mathrm{~K}$ of $T_{\mathrm{M}}$ (Figure 5) and the resultant PDFs were used to refine $8 \times 8 \times 8$ supercell models of the rocksalt structure (Figure 6). The models at the highest temperatures showed an increasing silver-ion occupation of $\left(\frac{1}{4}, \frac{1}{4}, \frac{1}{4}\right)$ interstitial sites, with a few $\%$ occupancy just below $T_{\mathrm{M}}$ and supporting the Frenkel mechanism for ionic conductivity in AgBr. A follow-up experiment [92], using a very stable and precise furnace, measured total scattering data to within $0.3 \mathrm{~K}$ of $T_{\mathrm{M}}$. These data were analysed using RMC, although in this instance the model was refined to structure factor data, leading to very similar structural conclusions to those of [50]. This work on $\mathrm{AgBr}$ spawned a number of similar studies on silver and copper superionic conductors [93] such as $\alpha$-AgI [94], $\mathrm{CuBr}$ [95] and $\mathrm{Ag}_{2} \mathrm{Te}$ [96] and provided the basis for RMC analysis of total scattering from the more technologically relevant oxide-ion conductors (e.g. [97]), although these later studies used the RMCProfile program described below.

It was soon realised that the PDF alone wouldn't always constrain a crystalline supercell structure well because it only contained information on the distances between atom pairs and information on the crystal lattice and average atom positions isn't directly encoded into the PDF. Furthermore, the total scattering structure factor, when calculated from the partial PDFs determined from a box of atoms (via Equations 12, 9 and 8) also only directs the relative atom pair distances over the distances within the supercell box. However this 'average' structural information is available in the Bragg powder pattern through the Bragg peak positions and intensities and it is straightforward to calculate these from the supercell model. This additional calculation was incorporated into the program RMCProfile [51] and this has become the RMC program of choice for crystalline materials.

The cost function in RMCProfile is made up of a weighted sum of a number of different cost functions

$$
\chi_{\mathrm{RMC}}^{2}=\sum_{j} \chi_{j}^{2}
$$

where

$$
\chi_{j}^{2}=\sum_{i}\left[f_{\text {calc }}\left(x_{i}\right)-f_{\text {expt }}\left(x_{i}\right)\right]^{2} / \sigma^{2}\left(x_{i}\right)
$$

The 'calc' and 'expt' subscripts refer to the calculated and experimental versions of function $f(x)$. $f(x)$ could be the total scattering structure factor, $F(Q)$, the PDF, 


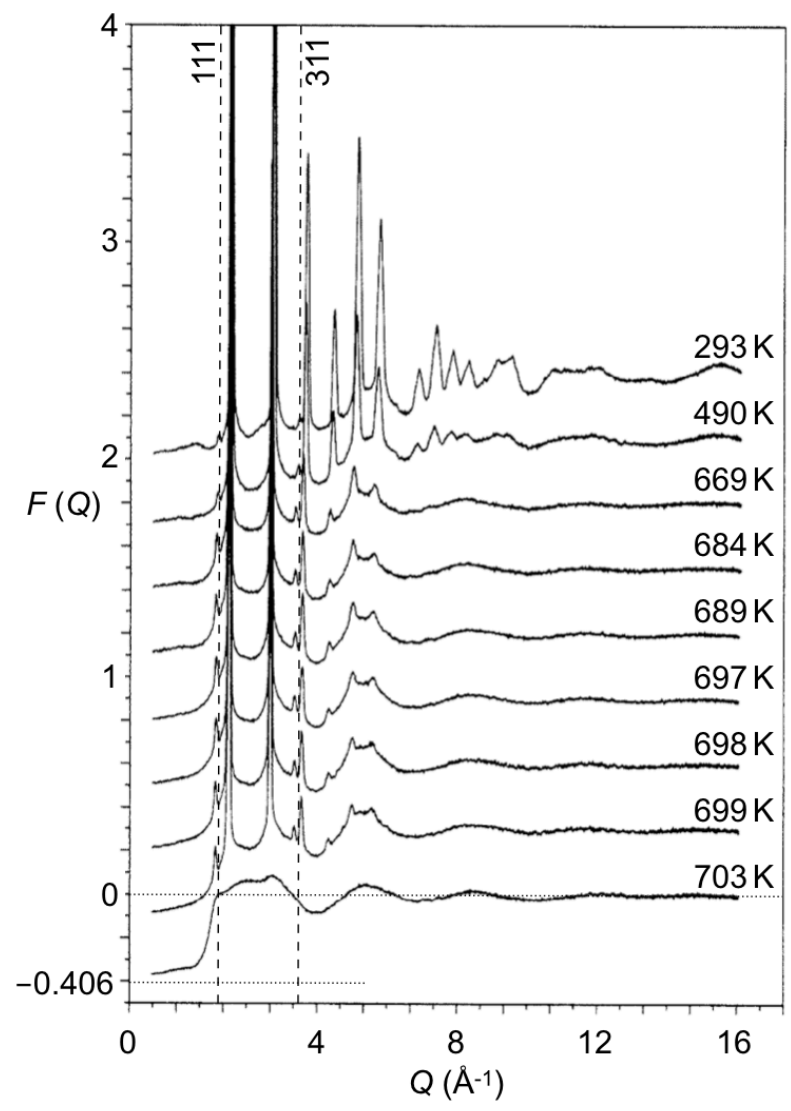

Figure 5. Neutron total scattering structure factor of molten $\mathrm{AgBr}$ at $703 \mathrm{~K}$, and of $\mathrm{AgBr}$ powder at a number of temperatures below $T_{\mathrm{M}}=701 \mathrm{~K}$. Each successive temperature has been offset vertically by 0.3 . Vertical dashed lines correspond to the positions of the 111 and 311 Bragg reflections at $293 \mathrm{~K}$. The structure factor for these reflections in the rocksalt structure is given by the difference between the $\mathrm{Ag}$ and $\mathrm{Br}$ neutron scattering lengths $\left(\bar{b}_{\mathrm{Ag}}=5.92 \mathrm{fm}, \bar{b}_{\mathrm{Br}}=6.80 \mathrm{fm}\right)$. These peaks increase in intensity as temperature is increased because the increased thermal disorder of the silver atoms effectively increases this scattering length difference especially as a growing percentage of the silver atoms moves into tetrahedral interstitial sites. The horizontal dotted lines give the theoretical limiting values for the molten $\operatorname{AgBr} F(Q)$ defined by Equation 21. (Adapted from [50] and reproduced with permission. Copyright IOP Publishing. All rights reserved.) 


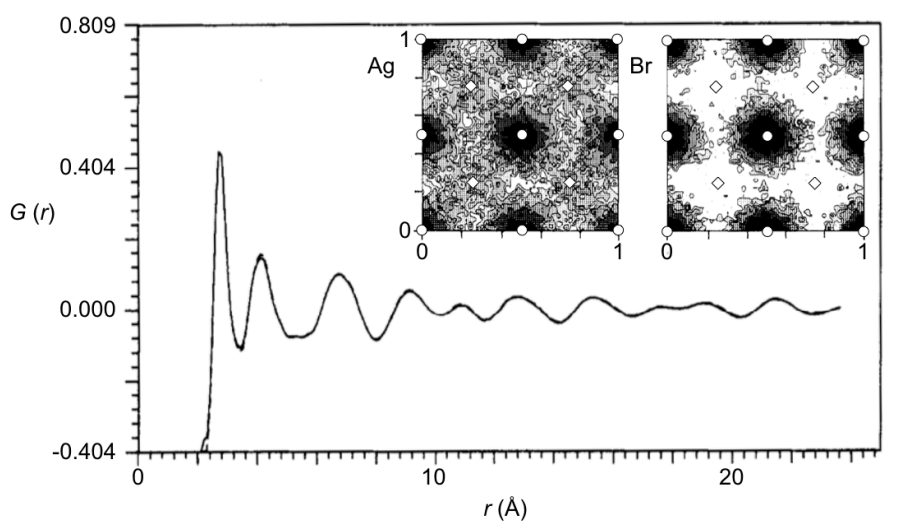

Figure 6. RMC fit to a neutron PDF of $\mathrm{AgBr}$ at $699 \mathrm{~K}, 2 \mathrm{~K}$ below $T_{\mathrm{M}}$. Dashed and full lines correspond to the experimental and calculated functions, respectively. The $y$-axis labels emphasise the limiting values for this function, as given by Equation 22. The inset shows the density distribution for silver and bromide ions in the RMC-refined model folded into a single unit cell and viewed down a 100-direction. In this projection the octahedral rocksalt positions for both atom types are labelled with white circles and the tetrahedral interstices with white diamonds. (Adapted from [50] and reproduced with permission. Copyright IOP Publishing. All rights reserved.)

$G(r)$, the Bragg profile, $I(t)$, or a restraint on the model such as a requirement for atoms to follow simple bonding potential functions. $\sigma\left(x_{i}\right)$ is the weighting of point $x_{i}$ related to its measurement error, although in practice RMCProfile usually uses a single overall weighting parameter for each function, $f$. Furthermore, although for ideal data with similar statistical precision it might be possible to assign the same weighting parameter to each experimental function, again in practice, their relative weights are adjusted to give greater prominence to the better quality data by requiring a closer agreement with these data, with their overall magnitudes chosen to give an acceptable proportion of accepted to rejected moves within the RMCProfile minimisation.

In addition to the random atom moves described above, atom pairs can be swapped (to investigate site disorder), neighbouring atoms can be required to remain within certain distance bounds (to maintain network topologies or molecular fragments) and magnetic spins can be rotated (to investigate magnetic structures, see Section 3.5).

The finite size of the RMC supercell model imposes a restriction on the form of $F(Q)$ in Equation 24 because it is only sensible to calculate the PDF out to distances that correspond to half the distance of the smallest dimension of the model, $L$. As the longer distances are not accessed, the higher frequency components in $F_{\text {calc }}(Q)$ are not calculated and so the $\chi^{2}$ in Equation 24 for the structure factor compares $F_{\text {calc }}(Q)$ with $F_{\text {expt }}(Q)$ convoluted with a box function of size $L / 2$.

The calculated Bragg profile function, typically as a function of neutron time-offlight, $t$, for spallation neutron source data, is given by

$$
I_{\text {calc }}\left(t_{i}\right)=\sum_{h k l} L_{h k l}|F(h k l)|^{2} R_{h k l}\left(t_{i}-t_{h k l}\right)+B\left(t_{i}\right)
$$

where $L_{h k l}, F(h k l)$ and $R_{h k l}\left(t_{i}-t_{h k l}\right)$ are the Lorentz factor, the structure factor and the peak shape of the $h k l$ reflection, respectively. $B\left(t_{i}\right)$ is the diffraction pattern background and an additional scale factor is usually applied before comparing to the experimental data. The values used to determine the scale factor, background coefficients and peak shape are obtained from a separate Rietveld refinement of the Bragg 

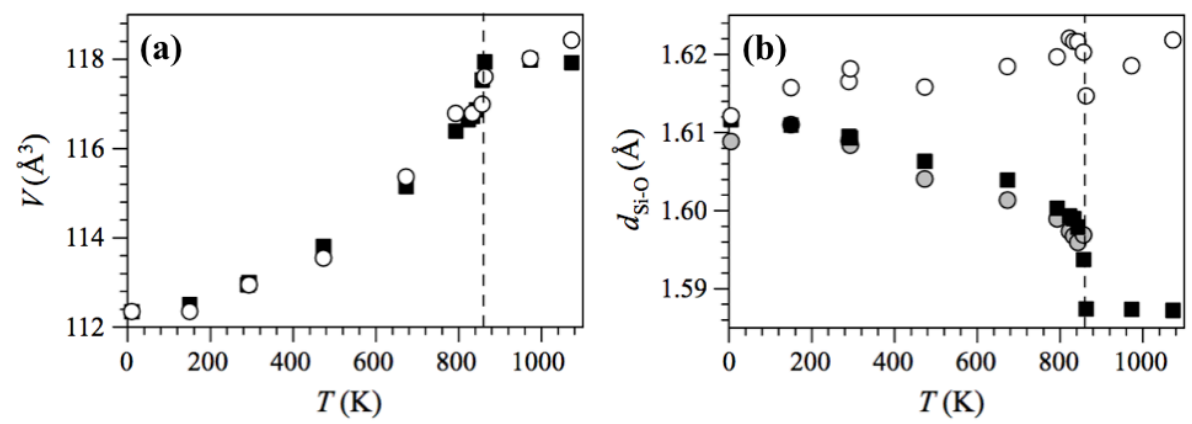

Figure 7. Temperature dependence of (a) the unit cell volume and (b) Si-O distance in quartz. The vertical dashed line marks the transition between the low-temperature $\alpha$-phase and high-temperature $\beta$-phase. The data in (a) are obtained from a Rietveld refinement of the Bragg profile and from the cube of the position of a peak in the PDF around $17 \AA$ that was scaled to coincide with the Rietveld-refined volume at $20 \mathrm{~K}$ (filled squares and open circles, respectively). In (b) the open circles are the average values of the instantaneous $\mathrm{Si}-\mathrm{O}$ bond length from the RMC model (i.e. $\langle\mathrm{Si}-\mathrm{O}\rangle$ ) which also corresponds to the position of the lowest- $r$ peak in the PDFs. The black squares and grey circles are the distance between average Si and O positions (i.e. $\langle\mathrm{Si}\rangle-\langle\mathrm{O}\rangle)$ from the Rietveld and RMC-refined models, respectively. These plots show how an RMC model can capture distinct local structure within a model that is simultaneously consistent with the average structure. (Reproduced from [101] with permission. Copyright IOP Publishing. All rights reserved.)

powder profile using a program such as GSAS-EXPGUI $[98,99]$. The Rietveld refined average structure is also used to define the positions of the Bragg peaks, $t_{h k l}$, and is used as the basis for the starting supercell model structure. As a result RMCProfile can be considered as a refinement program; a supercell of the average structure is refined to account for local variations that are encoded in the total scattering data.

The first study using RMC modelling where explicit account was taken of the Bragg peaks [100] was an investigation of the $\alpha-\beta$ phase transition of quartz [101,102]. This preceded the RMCProfile code and refined the supercell structure to the neutron PDF, $F(Q)$ and Bragg intensities, having first used the Pawley method [103] to extract the Bragg intensities from the experimental powder pattern. Restraints [104] were also used to maintain the topology of the crystal structure and prevent atoms from moving past each other and disrupting the overall structure of the network of corner-linked $\mathrm{SiO}_{4}$ tetrahedra. This work was the forerunner of a large number of RMCProfile analyses of phase transitions that have been used to show how local structure may or may not conform to the expected norms of classically-described phase transitions (see, for example, Section 4.1.1). The research in these papers highlighted how the local disorder begins at temperatures far below the $\alpha-\beta$ transition temperature (see also [105] for the implication of this on the piezoelectric properties of quartz) and that some features, such as the $\mathrm{Si}-\mathrm{O}$ bond length and other measures of thermal dynamic disorder, are largely insensitive to the phase transition (Figure 7). Others, such as the distribution of $\mathrm{Si}-\mathrm{Si}-\mathrm{Si}$ bond angles, change discontinuously at $T_{\mathrm{c}}$ (Figure 8 ), as does the overall average structure as determined from the RMC-refined supercell model.

The molecular disorder in the high-temperature phase of $\mathrm{SF}_{6}$ was used for the first demonstration of the RMCProfile code [106], followed by some interesting work on the negative thermal expansion (NTE) material $\mathrm{ZrW}_{2} \mathrm{O}_{8}[107,108] . \mathrm{ZrW}_{2} \mathrm{O}_{8}$ has a cubic structure which contracts for over $1000 \mathrm{~K}$ and with a particularly large negative thermal expansion coefficient of $-9.1 \mathrm{MK}^{-1}$ below room temperature [109]. The RMCProfile refinements supported an NTE interpretation based on flexibly linked rigid $\mathrm{ZrO}_{6}$ and $\mathrm{WO}_{4}$ polyhedral units within rigid unit mode theory [110]. The magnitude of the effect is large because each $\mathrm{WO}_{4}$ tetrahedron is only corner-linked to three 

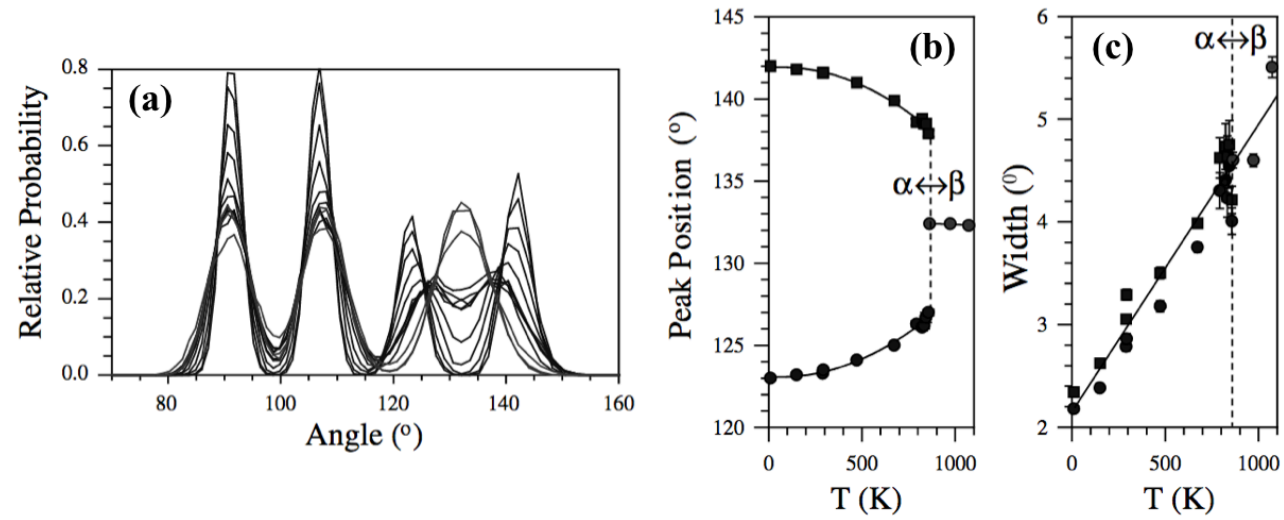

Figure 8. Temperature dependence of the $\mathrm{Si}-\mathrm{Si}-\mathrm{Si}$ bond angle distribution obtained from the RMC-refined models of quartz. (a) shows the overall distribution for temperatures between $20 \mathrm{~K}$ (four sharp peaks) and $1073 \mathrm{~K}$ (three broad peaks). The change from a four- to three-peaked distribution corresponds to the $\alpha-\beta$ phase transition as seen in (b) which plots the peak positions of the two higher-angled peaks that merge to form a single peak in the $\beta$-phase. (c) shows that the widths of these two peaks increase continuously through the phase transition, indicating that the phase transition is largely ignored by the thermal disorder. (Reproduced from [101] with permission. Copyright IOP Publishing. All rights reserved.)

neighbouring $\mathrm{ZrO}_{6}$ octahedra with the fourth oxygen atom non-bridging. This enables the $\mathrm{WO}_{4}$ tetrahedra to move substantially without a large energy penalty and the greater this movement the more the network structure contracts overall. Just before this RMCprofile study, an interpretation of XAFS measurements supported a stiffer linkage between $\mathrm{WO}_{4}$ and $\mathrm{ZrO}_{6}$ units [111]. In order to fit the XAFS data, the width of the $\mathrm{Zr} \cdots \mathrm{W}$ 'bond' refined to values that were as narrow as those of the (rigid) $\mathrm{W}-\mathrm{O}$ and $\mathrm{Zr}-\mathrm{O}$ bonds. A detailed analysis of the RMCprofile models showed that the structural model used in [111], by relying on the average 'crystallographic' structure, was slightly too simplistic. For example, the local distributions of bond angles, and how these distributions changed with temperature, would have a large impact on the multiple scattering corrections required for the XAFS data interpretation especially when the $\mathrm{Zr}-\mathrm{O}-\mathrm{W}$ bond angle is very close to $180^{\circ}$ (Figure 9) [108].

Another pair of early RMCProfile studies investigated how one structure, that of $\mathrm{Ag}_{3}\left[\mathrm{Co}(\mathrm{CN})_{6}\right][112]$, accommodated colossal thermal expansion [113], whilst the response of isostructural $\mathrm{H}_{3}\left[\mathrm{Co}(\mathrm{CN})_{6}\right]$ [114] was much more modest. The structure of $\mathrm{Ag}_{3}\left[\mathrm{Co}(\mathrm{CN})_{6}\right]$ consists of $\simeq 10 \AA$ long $\mathrm{Co}-\mathrm{CN}-\mathrm{Ag}-\mathrm{NC}-\mathrm{Co}$ linkages joining $\mathrm{CoC}_{6}$ octahedra to form three interpenetrating distorted cubic networks with Co atoms on the cube corners and $\mathrm{Co}-\mathrm{CN}-\mathrm{Ag}-\mathrm{NC}-\mathrm{Co}$ along each cube edge. The structure is trigonal and the interpenetrating networks are arranged to give alternating layers of $\mathrm{Ag}^{+}$and $\left[\mathrm{Co}(\mathrm{CN})_{6}\right]^{3-}$ ions perpendicular to the unique trigonal $c$-axis with the $\mathrm{Co}-\mathrm{CN}-\mathrm{Ag}-$ $\mathrm{NC}-\mathrm{Co}$ linkages aligned along $\langle 101\rangle$-directions. $\mathrm{Ag}_{3}\left[\mathrm{Co}(\mathrm{CN})_{6}\right]$ behaves like collapsible lattice fencing in response to temperature whereby the $c$-axis contracts as the $a$-axes expand. The key to this mechanism - and its surprisingly large magnitude - is the weak argentophilic $\mathrm{Ag} \cdots \mathrm{Ag}$ bonding within the $\mathrm{Ag}^{+}$layers which link the interpenetrating networks together, tying the lattice fencing together in its storage condition. At low temperatures this bonding produces a (relatively) long $c$-axis as the $a$ - $b$ layers perpendicular to the $c$-axis are kept compact. However, on heating the weak bonds are readily broken (the lattice fencing bundle is untied) so that the layers expand substantially (positive thermal expansion, PTE) and this is transmitted through the largely non-expanding $\mathrm{Co}-\mathrm{CN}-\mathrm{Ag}-\mathrm{NC}-\mathrm{Co}$ linkages to produce a colossal NTE response per- 
(a)

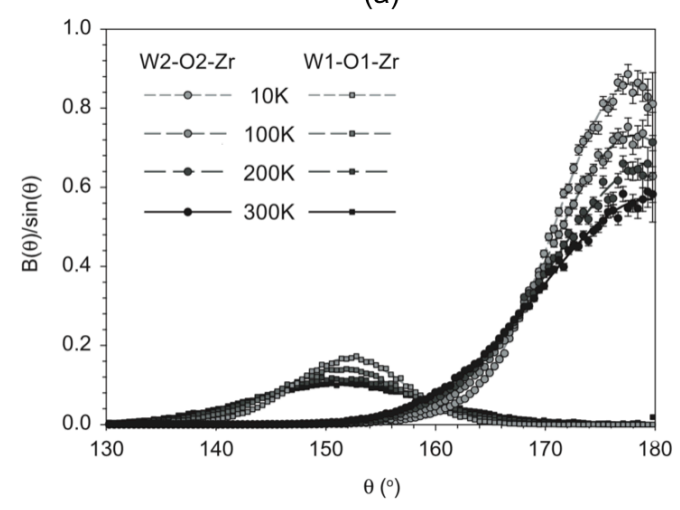

(b)

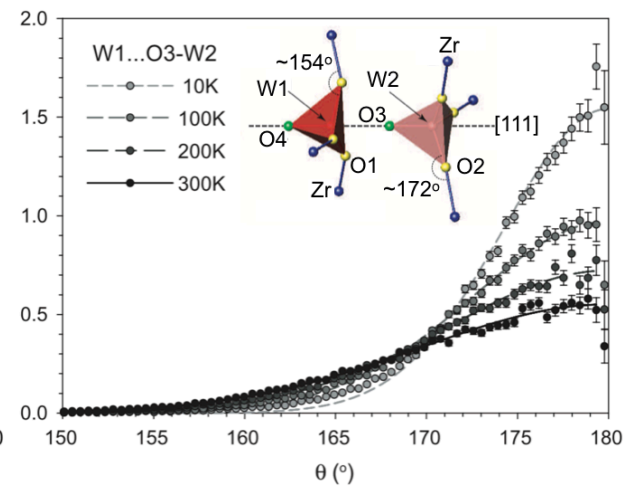

Figure 9. Key bond angle distributions from RMCProfile models of $\mathrm{ZrW}_{2} \mathrm{O}_{8}$ at four temperatures. The distributions are divided by $\sin (\theta)$ to capture the behaviour close to $180^{\circ}$ correctly. (a) Note that the peak of the $\mathrm{W} 2-\mathrm{O} 2-\mathrm{Zr}$ distribution, i.e. $\left\langle B_{\mathrm{W} 2-\mathrm{O} 2-\mathrm{Zr}}(\theta) / \sin (\theta)\right\rangle$, moves closer to $180^{\circ}$ as temperature increases (from $177.7^{\circ}$ at $10 \mathrm{~K}$ to $179.5^{\circ}$ at $\left.300 \mathrm{~K}\right)$ whereas the $\langle\mathrm{W} 2\rangle-\langle\mathrm{O} 2\rangle-\langle\mathrm{Zr}\rangle$ bond angle from Rietveld refinements decreases (from $172.4^{\circ}$ at $10 \mathrm{~K}$ to $171.5^{\circ}$ at $300 \mathrm{~K}$ ). (b) In contrast the non-bonded W1 $\cdots \mathrm{O} 3-\mathrm{W} 2$ bond angle distributions remain centred on $180^{\circ}$, albeit significantly increasing in width with increasing temperature. (Adapted from [108] and reproduced with permission. Copyright IOP Publishing. All rights reserved.)

pendicular to the layers. The magnitude of the effect is large, with thermal expansion coefficients of $\pm 130 \times 10^{-6} \mathrm{~K}^{-1}$ over a wide temperature range.

It was the nature of the temperature response of the $\mathrm{Co}-\mathrm{CN}-\mathrm{Ag}-\mathrm{NC}-\mathrm{Co}$ linkages that was the primary focus of the RMCProfile study. What was found was that although the individual average bond lengths along the linkage increased with increasing temperature the overall expansion of the linkage was very small. This apparent discrepancy was understood through the NTE effect of the transverse vibrational motions of the $\mathrm{C}$ and $\mathrm{N}$ atoms, with pronounced changes in the widths of the bond-angle distributions and, in the case of the $\mathrm{C}-\mathrm{N}-\mathrm{Ag}$ bond angle, movement of the mean position away from $180^{\circ}$ on heating. One interesting observation was that the flexibility of this linkage not only limited the overall Co $\cdots$ Co expansion but also was able to preserve the $\left[\mathrm{CoC}_{6}\right]$ geometries; $\left[\mathrm{CoC}_{6}\right]$ translations and whole-body rotations were more prevalent than $\mathrm{C}-\mathrm{Co}-\mathrm{C}$ bond-bending and $\mathrm{Co}-\mathrm{C}$ bond-stretching deformations.

$\mathrm{H}_{3}\left[\mathrm{Co}(\mathrm{CN})_{6}\right]$ is isostructural with $\mathrm{Ag}_{3}\left[\mathrm{Co}(\mathrm{CN})_{6}\right]$ if the $\mathrm{H}$-atom is centred between the neighbouring $\mathrm{N}$-atoms in the $\mathrm{Co}-\mathrm{CN}-\mathrm{H}-\mathrm{NC}-\mathrm{Co}$ linkage, either through the central position being the preferred location, or the $-\mathrm{N}-\mathrm{H} \cdots \mathrm{N}-$ off-centring producing a centred distribution on average. An overall off-centring would lead to a symmetry lowering of the structure. Differentiating between these options is an old problem [115] ${ }^{11}$ and one that is still relevant given the ongoing interest in short hydrogen bonds; the $2.6 \AA$ $\mathrm{N} \cdots \mathrm{N}$ separation in $\mathrm{H}_{3}\left[\mathrm{Co}(\mathrm{CN})_{6}\right]$ is at the critical distance below and above which the proton resides in a single and double well potential, respectively (e.g. [117]). The RMCProfile study [114] was based on neutron total scattering data from a deuterated sample and showed a transition between unimodal and bimodal character in the $-\mathrm{N} \cdots \mathrm{D} \cdots \mathrm{N}-$ motif on heating. This suggests that the structure has a covalent 'network' character at low temperatures and then switches to a more molecular arrangement at room temperature, albeit with the average structure still maintaining the same crystal symmetry.

This has important implications for the thermal expansion behaviour. The lin-

${ }^{11}$ Linus Pauling's notebook from the time indicates that this paper was written in a two-week period while Linus and his son Peter were at home over Christmas and New Year [116]. 
ear thermal expansion coefficients are much more modest than those observed for $\mathrm{Ag}_{3}\left[\mathrm{Co}(\mathrm{CN})_{6}\right]$, with values in the range $-15 \times 10^{-6}<\alpha<+20 \times 10^{-6} \mathrm{~K}^{-1}$, because the $\mathrm{H}^{+}$cannot act to 'tie' the structure up at low temperature in the way that the bonding within the layers of $\mathrm{Ag}^{+}$do. There is also a marked difference between $\mathrm{H}_{3}\left[\mathrm{Co}(\mathrm{CN})_{6}\right]$ and $\mathrm{D}_{3}\left[\mathrm{Co}(\mathrm{CN})_{6}\right]$, with the magnitude of NTE along the unique axis significantly greater in the hydrogenous compound at the lower temperatures. This can be explained by the relative vibrational energies of $\mathrm{N}-\mathrm{D}$ and $\mathrm{N}-\mathrm{H}$; the lower value for the $\mathrm{N}-\mathrm{D}$ stretching frequency allows the cross-over from unimodal to bimodal linkages at a lower temperature. The unimodal arrangement will show very small or negative thermal expansion along the linkages and will couple the PTE within the plane to a NTE perpendicular to the plane whereas the bimodal arrangement will produce positive thermal expansion along the linkages and break the PTE-NTE coupling between the perpendicular directions in the structure.

These RMCProfile studies on unusual thermal expansion behaviour serve to demonstrate one of the key strengths of analysing total scattering with 'big box' refinement methods - the ability to reveal local structural disorder associated with dynamic processes. As I show in later examples, this strength has been used to reveal important structural detail in the characterisation of disorder, especially across phase transitions. It is probably unique as a method for producing experiment-based atomistic models that capture both the average (crystallographic) structure whilst simultaneously revealing how the local arrangements of atoms might deviate from this average structure.

\subsection{2. 'Small box' refinements and PDFgui}

The modern approach to this fitting method would typically use PDFgui [55], the graphical user interface for the enhanced $\mathrm{C}++$ version of the PDF fitting program PDFFIT [118]. PDFFIT is based on the methodology described by Kaplow and coworkers in the 1960's when fitting models to their PDF data from lead and cobalt [25,27] (see Section 2.3.2). As with RMCProfile, the principle is very straightforward. A small box of atoms, typically one unit cell, is used to calculate all the distances between atom pairs within a required $r$-range using periodic boundary conditions. These distances are then weighted by the appropriate scattering factors and broadened by convoluting with Gaussian functions with widths calculated from the atomic displacement parameters and an $r$-dependent term to account for local correlated motion. The calculated PDF is further modified by convoluting with a $\sin \left(Q_{\max } r\right) / r$ term to account for termination ripples arising from the finite range of the reciprocal-space data (i.e. the data only extend to $\left.Q_{\max }\right)$ and damped by a term $\exp \left(-\sigma_{Q}^{2} r^{2} / 2\right)$ to account for the resolution in $Q$-space. $\sigma_{Q}$ is either treated as a refineable parameter or determined from a measurement of a calibration standard. PDFgui allows the user to choose which parameters to refine and the refinement protocol follows a standard least-squares approach. The structural parameters that are refined are the same as would be refined in a typical Rietveld refinement: cell parameters, atom coordinates and displacement parameters etc. and the method is aptly described as a "real-space Rietveld" refinement technique.

The first use of PDFFIT was in a study of $\mathrm{LaMnO}_{3}$ using neutron PDF data measured at $20 \mathrm{~K}$ [119]. The authors made a careful comparison of the Jahn-Teller distortion of the $\mathrm{MnO}_{6}$ octahedra, comparing the size of the short, medium and long $\mathrm{Mn}-\mathrm{O}$ bonds obtained from Rietveld refinement and PDFFIT refinements over a short $(1.5 \AA<r<3.5 \AA)$ and longer $(1.5 \AA<r<15.5 \AA)$ distances. Good agreement was 
obtained from all three refinements showing that the behaviour seen in the average structure was replicated at the short-range. The study was followed up by a similar temperature-dependent study between $300 \mathrm{~K}$ and $1150 \mathrm{~K}$ [120]. They found that, when refining the structure to the PDF over the low- $r$ region, the Jahn-Teller distortion of the $\mathrm{MnO}_{6}$ octahedra observed in the low-temperature phase was retained for all temperatures, even through the transitions to higher symmetry average structures (see Figure 10(a) and (b)). In contrast, when refined to PDF data out to $20 \AA$ the structure reflected that of the average. This direct observation that the $\mathrm{MnO}_{6}$ octahedra remained distorted for all temperatures was an important observation for understanding the behaviour of orbital order within the perovskite manganites, but the paper also highlights an issue with the PDFFIT method. Because the refinement is of a small fragment of the structure which then decorates the lattice to obtain pair distances greater than the size of the fragment, if the fragment distorts in a way that is incompatible with the average lattice then different parts of the the calculated PDF pattern will attempt to refine the structure differently. The short-ranged data will produce the distorted structure whereas as the pair distances become progressively larger the structure will tend to the average. This is clearly seen in [120] which has a figure (part of which is reproduced in Figure 10(c)) showing how the Mn-O distances slowly morph from the more distinct Jahn-Teller distorted values (fitting to short distances) to all being more or less equivalent (when fitting out to $20 \AA$ ) at high temperature. This can be a good indicator of the behaviour of the structure, indeed in [120] the authors use these trends to identify ordered cluster sizes. It has been developed into the so-called 'box-car' approach (see, for example, [121]) whereby a fixed range of $r$-values are used to refine the structure with the range beginning at progressively larger values of $r$. However it is not always entirely satisfactory and I shall discuss this further in a later section when comparing analysis methods (see Section 3.6.2).

\subsection{Magnetic Total Scattering}

The theoretical basis for the interaction between the magnetic moment of the neutron and the magnetic moment of atoms was developed in the 1930's [122]. Around the same time experiments were carried out on the diffuse neutron scattering from paramagnetic material to deduce the nature of the neutron magnetic moment and magnetic form factor associated with the scattering. It was two papers by Shull and co-workers $[123,124]$ that showed structured diffuse neutron scattering (i.e. scattering that was neither following the magnetic form factor shape nor sharply peaked at Bragg positions) from $\mathrm{MnO}$ at room temperature suggesting that 'the magnetic moments are not completely randomly arranged, but that there exists a short-range magnetic order with neighboring moments tending to be aligned' [124]. Although their work used powdered samples, most of the magnetic diffuse neutron scattering studies that followed preferred to use single crystals, especially when using polarised neutron beams (see [125], for example). Furthermore, where possible, the magnetic diffuse scattering is separated experimentally from other scattering (such as the nuclear Bragg and diffuse scattering) before analysis of the magnetic structure. Typically the structured magnetic contribution would be isolated either by subtracting data above a magnetic phase transition from the data below or by using the more time-consuming neutron polarisation techniques. To my knowledge there are no reports of analysis of the combined total scattering (i.e nuclear plus magnetic, diffuse plus Bragg scattering) without 
(a)

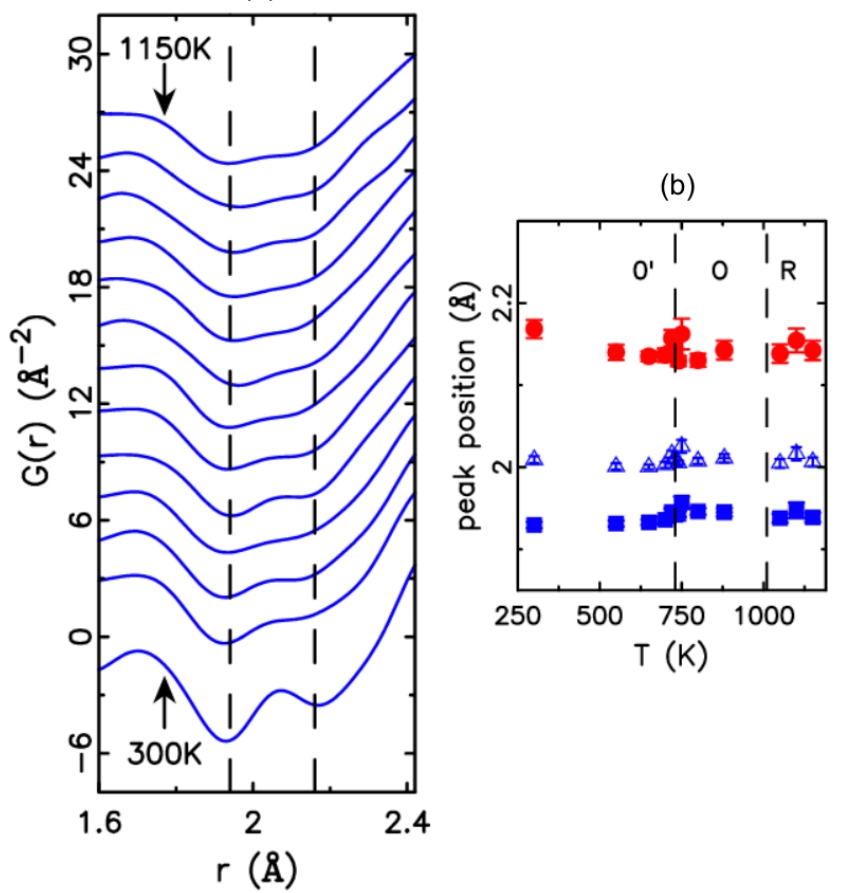

(c)

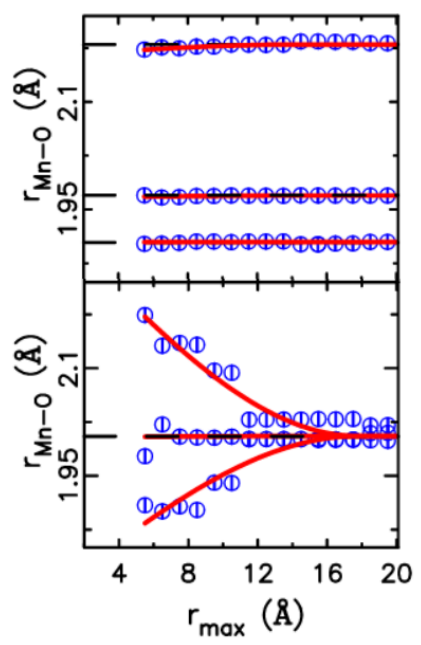

Figure 10. Neutron pair distribution functions from $\mathrm{LaMnO}_{3}$ measured at a number of temperatures. (a) Low- $r$ portion of the PDF showing the (negative) Mn-O peaks corresponding to the 'short' and 'long' bonds in the $\mathrm{MnO}_{6}$ octahedra (dashed vertical lines). (b) Results from fitting Gaussians to the two peaks in (a), including the weighted average positions (open triangles, the fits were constrained to have twice as many 'short' bonds as 'long'). The refined 'short' bond Gaussians were broader than those of the 'long' bond most likely because they included a contribution from the 'medium' bonds that are expected in the $\mathrm{O}^{\prime}$ structure but not resolved in the PDF. The vertical dashed lines correspond to the phase transitions between the two orthorhombic $\left(\mathrm{O}^{\prime}\right.$ and $\mathrm{O})$ and rhombohedral (R) phases. (c) $\mathrm{Mn}-\mathrm{O}$ bond lengths obtained from a refinement of the structure to the PDF between $1.5 \AA$ and $r_{\max }$ at $300 \mathrm{~K}$ (upper plot) and $1100 \mathrm{~K}$ (lower plot). Here three distinct Mn-O bond lengths are obtained at both temperatures when $r_{\max }$ is small; as $r_{\max }$ increases the refined bond lengths tend towards the Rietveld refined values (given by horizontal dashed lines in both plots). (Reprinted from figures with permission from [120]. Copyright 2005 by the American Physical Society.) 
using experimental separation methods. ${ }^{12}$

It is however possible to denote magnetic total scattering as the neutron diffuse and Bragg scattering which has a magnetic origin and arises from the orientation of magnetic moments on a (fixed) arrangement of magnetic atoms. The magnetic total scattering formalism, in terms of spin-spin correlations, was first set out in 1964 in a paper by Blech and Averbach [127]. They used the Fourier transform of the magnetic total scattering to investigate the likely spin arrangements in $\mathrm{MnO}$ above the Néel temperature; local correlations between magnetic moments exist at temperatures well above $T_{\mathrm{N}}$ in $\mathrm{MnO}$ [128]. This formalism was later incorporated into an RMC code and used to study the magnetic structure of amorphous materials [129]. The key amorphous material for developing the magnetic RMC methods was the metallic glass $\mathrm{Dy}_{7} \mathrm{Ni}_{3}$, the total scattering from which had been measured [130] for 4 different isotopic compositions, ${ }^{\mathrm{N}} \mathrm{Dy}_{7}{ }^{\mathrm{N}} \mathrm{Ni}_{3},{ }^{\mathrm{N}} \mathrm{Dy}_{7}{ }^{0} \mathrm{Ni}_{3},{ }^{0} \mathrm{Dy}_{7}{ }^{\mathrm{N}} \mathrm{Ni}_{3}$ and ${ }^{0} \mathrm{Dy}_{7}{ }^{0} \mathrm{Ni}_{3}$, where the superscripts $\mathrm{N}$ and 0 refer the the natural isotopic abundance and a ratio chosen such that the overall neutron scattering length is zero, respectively. (This is somewhat fortuitously possible for this metallic glass because there are isotopes of both $\mathrm{Dy}$ and $\mathrm{Ni}$ with positive and negative scattering lengths.) An atomistic model of the metallic glass was refined using the room temperature (nuclear) total scattering structure factors, ${ }^{\mathrm{NN}} F(Q),{ }^{\mathrm{N} 0} F(Q)$ and ${ }^{0 \mathrm{~N}} F(Q)$, obtained by subtracting the " 00 " total scattering data from that of each of the other three compositions, and then magnetic moments were added to the Dy atoms in the RMC model. Using the RMC modelling method, these moments were then rotated - while keeping the atoms fixed - until a good agreement with the ${ }^{0} \mathrm{Dy}_{7}{ }^{0} \mathrm{Ni}_{3}$ structure factor, ${ }^{00} F(Q)$, was obtained (which contained no signal from the atomic structure). The temperature dependence of the magnetic structure was then determined by refining the magnetic spin orientations to ${ }^{0} \mathrm{Dy}_{7}{ }^{0} \mathrm{Ni}_{3}$ total scattering data at temperatures below room temperature. The low-temperature spin-spin correlation function was then compared to what might be expected for a RudermanKittel-Kasuya-Yoshida (RKKY) indirect exchange interaction operating on the Dy spins separated by the experimentally determined distance [131].

Similar methods were applied in a study of the ordered magnetic structure of $\mathrm{MnO}$ at low-temperature [126], a system very different to the disordered magnetic metallic glasses described above. $\mathrm{MnO}$, despite being thought of as an archetypal antiferromagnet and the first material used to demonstrate the ability of neutron diffraction to observe antiferromagnetism, has some unresolved anomalies in its magnetic structure. Below the Néel temperature the cubic rocksalt atomic structure distorts into a rhombohedral arrangement and the Mn moments align ferromagnetically within planes perpendicular to the rhombohedral unique axis and antiferromagnetically between successive planes. It is however not possible to deduce the direction of the spins within these (111) planes from neutron powder diffraction, and untwinned single crystal samples in the rhombohedral phase are virtually impossible to obtain $[132,133]$. Furthermore, this arrangement of spins is incompatible with rhombohedral symmetry and the true symmetry must be lower [134]. Goodwin et al [126] measured total scattering data from $\mathrm{MnO}$ powder at $10 \mathrm{~K}$ and analysed the resultant total scattering structure factor and Bragg profile using RMCProfile [51]. The two main advantages of this approach were that the Bragg and diffuse scattering were analysed simultaneously and, by measuring the data to high- $Q$, accurate positions of the magnetic atoms were obtained. In addition, apart from the overall shape and size of the model, the model

\footnotetext{
${ }^{12}$ With the possible exception of the work on the low temperature structure of MnO [126] described below, although even in this example the atomic and magnetic structures are highly ordered and the total scattering data contained very limited diffuse scattering.
} 
is not constrained to obey a specific symmetry and any symmetry that develops in the refined models will have been directed by the data alone. All models converged to the expected ferromagnetic sheets parallel to (111) stacked antiferromagnetically along [111], although different RMCProfile runs produced different directions for the spins within the (111) planes. Additionally, there was a weak preference for the spins to align along $\langle 11 \overline{2}\rangle$ axes and modulated out-of-plane spin components and atom displacements consistent with space group $C 2$, albeit with Bragg peak splittings smaller than the instrumental resolution and superlattice peaks which were too small to be observed conclusively.

This work using RMC led to the development of the program spinvert [135], a dedicated RMC-based program for refining disordered magnetic structures using magnetic diffuse scattering data. This has had considerable success for a wide range of systems using data from powdered [136] and single crystal samples [128]. Although spinvert does not strictly use magnetic total scattering, when applied to disordered systems it effectively is as the magnetic Bragg component is zero. It also fits to the magnetic total scattering in reciprocal space and the magnetic PDF is by-passed in the refinement. This is for good reason. The magnetic contribution to neutron scattering is modified by the magnetic form factor which decays fairly rapidly as $Q$ increases. The Fourier transform of a magnetic signal of this form produces broadened features in the PDF and a contribution at low- $r$, a part of the PDF where data normalisation uncertainties often dominate. These issues were somewhat mitigated against in the initial experimental work on $\mathrm{Dy}_{7} \mathrm{Ni}_{3}$ where a magnetic difference PDF ( $\mathrm{m} \Delta \mathrm{PDF}$ ) was calculated as the Fourier transform of ${ }^{00} \Delta F(Q), \Delta F(Q)$ being the difference between $F(Q)$ measured at low-temperature and high-temperature [130].

The form and theory of mPDF's have been investigated in more detail by Frandsen and colleagues $[137,138]$. In their formalism the magnetic total scattering structure factor is divided by the magnetic self-scattering (calculated from the neutron magnetic form factors) prior to Fourier transformation, analogous to dividing X-ray structure factors by a sharpening factor. The resultant $\mathrm{mPDF}$ has been analysed in a number of systems, although to do so the atomic and magnetic contributions to the combined (nuclear and magnetic) PDF need to be separated, often by fitting the combined PDF (or the combined total scattering) with the atomic structure and using the residual to obtain the mPDF.

The first application of mPDF techniques was-perhaps unsurprisingly given the history of magnetic total scattering outlined above - to the low-temperature magnetic structure of $\mathrm{MnO}$ [138]. Very good fits to the $\mathrm{mPDF}$ data from $\mathrm{MnO}$ at $5 \mathrm{~K}$ were obtained using a magnetic structural model based on that proposed previously [126], although there were differences in the detail, with suggestions of a higher symmetry $C 2 / m$ monoclinic space group and the possibility of the Mn moments within the (111) sheets favouring a different alignment direction. The similarity of the results with those from RMCProfile confirmed the robustness of the mPDF method, whereas the differences highlight the difficulty in obtaining information on these subtle magnetic structural details when they cannot couple strongly to the neutron diffraction measurements. 


\subsection{Choosing the optimal radiation/instrumentation/analysis methodology}

At the start of this section (3.1), I stated that it was the establishment of four techniques - spallation neutron and X-ray synchrotron total scattering measurements and 'big'- and 'small'-box refinements - that have underpinned the extensive use of the PDF within crystallography today. Access to shorter-wavelengths at these central facilities have extended the $Q$-range of the total scattering data and improved the $r$-space resolution in the PDFs, thus increasing the effectiveness in more complex structural problems. This makes the methods more widely applicable and having established more user-friendly analysis tools the barriers to learning the methods have been significantly reduced. But how should the methods be compared and for a given problem which scattering probe and analysis method should one consider? This section aims to provide some guidance.

\subsubsection{X-ray or neutron total scattering?}

The considerations for making this choice for diffraction experiments are well-known. Relative scattering strengths prioritise neutrons over X-rays for hydrogen containing samples, light elements in the presence of heavy elements and for some neighbouring elements. X-rays are much more appropriate for small samples and when rapid data collections are required. An X-ray PDF might be determined from a few seconds' measurement on a sub-mm diameter capillary; the equivalent neutron PDF would require several hours of counting on a sample $\sim 1-2 \mathrm{~cm}^{3}$. However other 'traditional' distinctions become more nuanced when considering total scattering. The greater penetration of high-energy X-rays (relative to those of lower energy), coupled with smaller samples, means that the absorption of the sample is comparable for Xray and neutron measurements. There is also a need for rigorous data normalisation. This typically favours neutron measurements since absolute normalisation is possible using vanadium-niobium calibration, although the quality of the normalisation can be compromised when the samples contain light atoms such as hydrogen. It also might be helpful to exploit the more extreme relative X-ray scattering strengths in cases where the more even neutron scattering contrast is a hindrance. For example, metallic nanoparticles are often coated with organic material such that the number of atoms in the coating far exceeds those within a nanoparticle with a diameter of a few nanometres. In this case, especially if the nanoparticle contains heavy metals such as gold, an $\mathrm{X}$-ray PDF is going to be much more appropriate (unless the interest is in the coating or coating-nanoparticle surface interaction of course, although this would also be a challenging neutron experiment). Faster data collection and the potential for greater scattering contrast also means that in-situ experiments are also far more suited to $\mathrm{X}$-ray PDF measurements. The key thing is to calculate the expected partial PDF weighting factors for the X-ray (Equations 20 or 15) and neutron (Equation 11) PDFs before making the choice of scattering probe.

Although X-ray total scattering data can now be collected to high- $Q$, and values above $40 \AA^{-1}$ are in principle possible [78], the damping effect of the X-ray form factor on the signal means that high- $Q$ data above $\sim 30 \AA^{-1}$ are still a challenge with existing instrumentation. Area detectors restrict the maximum scattering angle available and if the X-ray energy is increased to counteract this limitation, the overall background tends to increase as the shielding becomes less effective. Correcting area detectors for background and other sources of noise is also a challenge at high- $Q$; very small 
variations in their response or other small glitches are amplified when the sample signal is minimal and these can be extremely difficult to account for satisfactorily. In contrast, high- $Q$ data are easily obtained from a well-designed powder diffractometer at a spallation neutron source which has a high flux of epithermal (short-wavelength) neutrons. The highest real-space resolution is routinely available from neutron total scattering. It may also be thought that the highest reciprocal-space resolution would be routinely available using synchrotron X-ray instruments, given the availability of highly parallel beams and detectors with very small pixels. However this is not always the case because to obtain the necessary $Q$-range for PDF means that the detectors are placed very close to the sample. In general the $Q$-space resolution for typical synchrotron Xray and spallation neutron total scattering diffractometers are comparable, although the neutron total scattering will include data from backward scattering detector banks with better resolution, and some instruments such as I15-1 (XPDF) include optional higher-resolution detection, should analysis of Bragg data alone be required.

Of course it could be that the answer is that X-ray and neutron total scattering are required. This is increasingly the response for those working on liquids and amorphous materials; X-ray plus neutron total scattering plus modelling is taking the place of the more established-but limited to specific materials - isotopic neutron scattering methods to obtain structural models. Some facilities now offer reciprocal arrangements where neutron and X-ray data can be collected via a single application process and this is being taken advantage of for crystallographic problems (see Sections 3.6.3 and 3.6.4 below).

\subsection{2. 'Big'- or 'small'-box refinement?}

Small-box PDF refinements have the advantages of being quick, both in terms of time to set up through PDFgui [55] and in computation time, and more tolerant of total scattering data quality. The physical part of the PDF used by these methods is usually very robust to most of the data normalisation issues outlined in earlier experimental sections, issues which often will introduce large systematic errors in the reciprocal space total scattering structure factor data. Hence a method that refines the model structure to the PDF alone and uses an arbitrary (fitted) scale factor for the calculated PDF bypasses many of the more serious problems that might occur with non-ideal data.

Big-box refinements, in contrast, are dependent on having absolutely normalised total scattering data with consistently normalised real and reciprocal space neutron and/or X-ray data (and Bragg peak profile data for the case of RMCProfile). This is because the atoms within the supercell model are free to disorder/order to adjust the overall intensities of the features (i.e. the scale factor) in the PDF data. This places a greater emphasis on obtaining good quality data, particularly reciprocal space data where a smoothly varying offset would give rise to a poor fit to $F(Q)$ even though this would only cause a problem in the PDF at low- $r$ which is unphysical and (rightly) ignored by small-box refinements. They therefore typically take longer to set up, require more attention to the data normalisation (for example, the ad-hoc approaches of data normalisation such as PDFgetX3 [139] and PDFgetN3 [140] that do not necessarily place the PDF data on an absolute scale or produce well-corrected structure factor data would not be appropriate in the majority of cases), and take much longer to run.

So, given that PDFgui is more straightforward, faster and requires simpler data processing, when might one consider running RMCProfile in preference to PDFgui? Very broadly, if the disorder is extensive within the crystal structure then RMCProfile 
would be more suitable, whereas if it is very short-ranged or gives rise to small distortions then PDFgui is appropriate. Structures that show large atom displacements, or atom diffusion, or where there is a need to show disorder beyond the size of the small-box models, are all better refined using RMCProfile. 'Big'-box models can also be straightforwardly linked to disorder models from molecular dynamics simulations [93]. RMCProfile can be used for all structure disorder problems, but it would be unnecessarily laborious to do so for the subset of structure disorder problems where PDFgui would excel. A benefit of big-box methods is that they are able to refine local disorder whilst also requiring that these disordered structural units pack together to produce a supercell structure that, when spatially averaged into the unit cell, is consistent with the average structure (i.e. the structure obtained from refining to the Bragg peaks alone). The PDFgui method doesn't impose this requirement on the refined local disordered structure and cannot be used to compare with the Bragg intensities. To account for this in scenarios where the local structure is very different from the average and where the PDF fit is only effective over a limited range of pair distances, PDFgui can be used to fit the data over different $r$-ranges or over a rolling small range of distances (a "box-car" compromise approach) to show how the local structure slowly morphs into the average (e.g. $[120,141])$.

\subsubsection{Hydrogen disorder in hydrogrossular}

Fully hydrated hydrogrossular, also known as katoite, is the end-member of the garnet series $\mathrm{Ca}_{3} \mathrm{Al}_{2}\left(\mathrm{SiO}_{4}\right)_{3-x}(\mathrm{OH})_{4 x}$ with $x=3$, i.e. $\mathrm{Ca}_{3} \mathrm{Al}_{2}\left[(\mathrm{OH})_{4}\right]_{3}$. The structure can be thought of as that of grossular garnet, but with every $\mathrm{SiO}_{4}$ tetrahedra within the structure replaced by $(\mathrm{OH})_{4}$ 'units' i.e. every $\mathrm{Si}$ atom has been removed and replaced by $4 \mathrm{H}$ which form $\mathrm{O}-\mathrm{H}$ bonds with the $\mathrm{O}$ atoms that were surrounding the $\mathrm{Si}$ atoms. Although this expands the lattice and the $\mathrm{H}$ atoms predominantly reside outside the $(\mathrm{Si})_{\text {vacancy }} \mathrm{O}_{4}$ tetrahedra, the average structure has a very short $\mathrm{H}-\mathrm{H}$ contacts indicating a likelihood of their correlated motion [142].

A total scattering study to investigate the nature of this possible correlated hydrogen motion within fully hydrated hydrogrossular clearly requires a measurement using neutrons to have any sensitivity to disordered hydrogen locations and the sample should also be perdeuterated to reduce the hydrogen incoherent scattering and improve data quality. However, the contribution to the PDF from other atom pairs, and particularly those with a low concentration (i.e. $\mathrm{Al}-\mathrm{Al}, \mathrm{Al}-\mathrm{Ca}$ and $\mathrm{Ca}-\mathrm{Ca}$ ), are relatively weakly weighted in the neutron PDF. The relative weightings are effectively reversed in the X-ray PDF; the weighting of all pairs involving hydrogen are reduced whereas those of all other atom pairs are increased. In view of this, a recent study [143] used a combination of neutron and X-ray total scattering (Figure 12) and RMCProfile to investigate the nature of deuterium disorder within katoite. It was anticipated that the X-ray data would constrain the overall garnet structure well whilst the neutron data would provide the necessary detail on the deuterium atom locations. Furthermore, given the extent of the deuterium atomic displacement parameters in the Rietveld-refined structure, RMCProfile was preferred to PDFgui as the analysis method.

This detailed total scattering/PDF analysis gave good agreement between the scattering functions calculated from the supercell model and the data. It showed that the D atoms were able to 'fit' within the structure rather straightforwardly and without any obvious need for correlated mutual avoidance; indeed if anything the shortest D-D contacts were even shorter than those determined from the average structure (Figure 


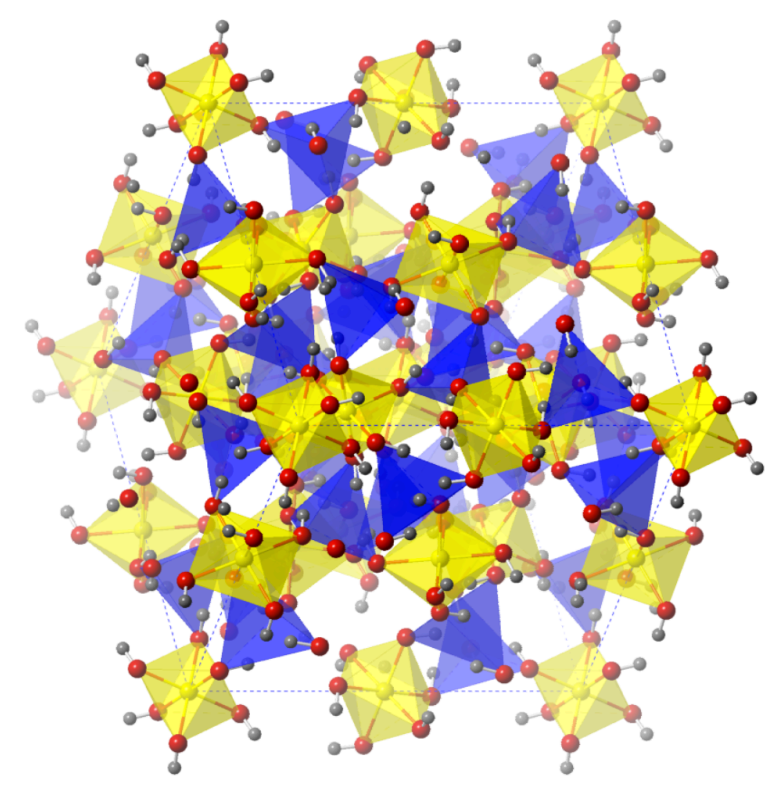

Figure 11. Crystal structure of hydrogrossular, $\mathrm{Ca}_{3} \mathrm{Al}_{2}\left[(\mathrm{OD})_{4}\right]_{3}$. Yellow and blue polyhedra correspond to $\mathrm{AlO}_{6}$ octahedra and $(\mathrm{Si}) \mathrm{O}_{4}$ tetrahedra, respectively, albeit with each $\mathrm{Si}$ atom in the grossular garnet structure replaced by four $\mathrm{D}$ atoms. $\mathrm{Al}, \mathrm{O}$ and $\mathrm{D}$ are shown as yellow, red and grey spheres, respectively; Ca atoms, which sit in the interstices of the corner-linked $\mathrm{AlO}_{6}-(\mathrm{Si}) \mathrm{O}_{4}$ network, have been omitted for clarity. (Using crystal structure data from [143].)

13). The neutron PDF provided a direct measurement of the length of the $\mathrm{O}-\mathrm{D}$ bond, showing that the Rietveld refined value was somewhat (artificially) shortened by the way the average structure model characterised the thermal motion, but other than this any local structure did not deviate from what might be anticipated from the average structure. (As a postscript, in this RMCProfile refinement the approximation given by Equation 20 was used to form the calculated X-ray PDF (see Figure 14). This $G^{X}(r)$ contained a clear peak at the O-D position even though no peak is observed in the $\mathrm{X}$-ray PDF data. This is a good example of where Equation 20 is less than ideal as the X-ray scattering factor for D-atoms reduces to zero much faster with increasing $Q$ than that of the overall scattering factor for hydrogrossular. This causes the O-D contribution to the PDF to broaden significantly, to the extent that the $\mathrm{O}-\mathrm{D}$ bond isn't observed in the data.)

\subsubsection{Orbital order/disorder in $\mathrm{LaMnO}_{3}$}

The behaviour of the Jahn-Teller distorted $\mathrm{MnO}_{6}$ octahedra in $\mathrm{LaMnO}_{3}$, as determined using PDFFIT refinements to neutron PDF data, has already been discussed (Section 3.4.2 above, [119]). A further investigation was carried out more recently using neutron and X-ray total scattering data analysed with a combination of RMCProfile and PDFgui refinements [144]. This study was also concerned with the nature of the orthorhombic $\left(O^{\prime}\right)$ to pseudocubic $(O)$ phase transition at $T_{\mathrm{JT}}=750 \mathrm{~K}$, widely viewed as a typical orbital order/disorder transition [145] whereby the Jahn-Teller distortions are retained across the transition but change from being fixed (below $T_{\mathrm{JT}}$ ) to fluctuating (above $T_{\mathrm{JT}}$ ). An alternative view based on a three-state Potts (3SP) model proposed that, although the low-temperature octahedral distortions were retained above $T_{\mathrm{JT}}$, the long $\mathrm{Mn}-\mathrm{O}$ bonds that reside within planes in the $O^{\prime}$ phase 

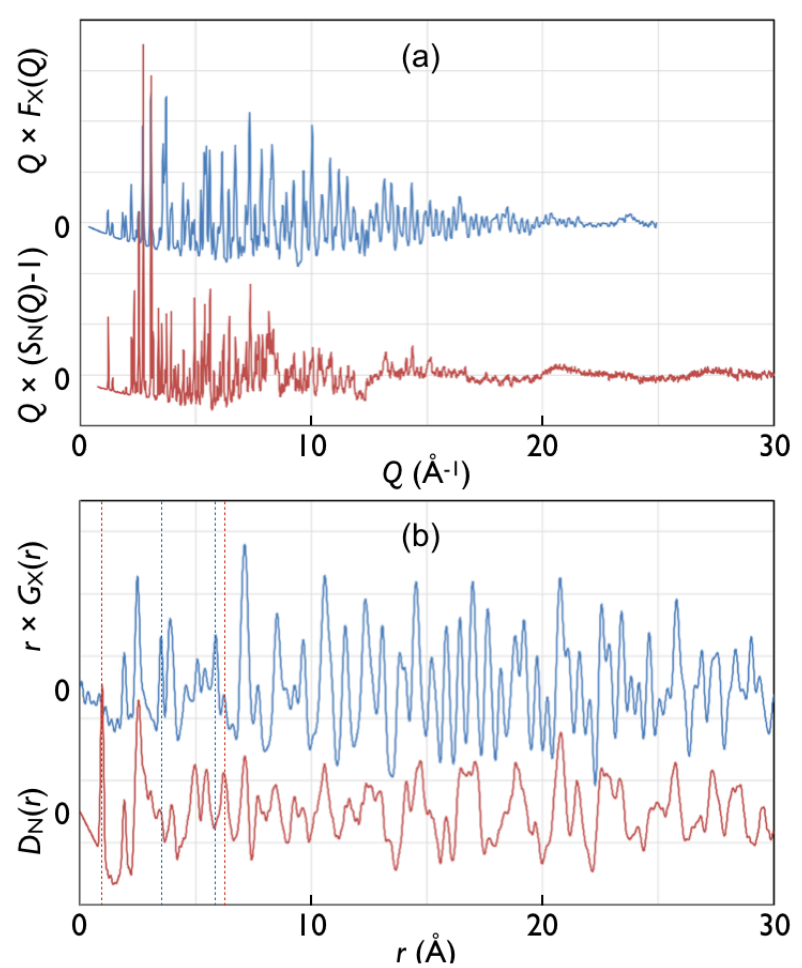

Figure 12. X-ray and neutron total scattering from hydrogrossular. (a) X-ray (upper curve) and neutron (lower curve) structure factors measured on XPDF and GEM, respectively. In this material the Bragg peaks persist to higher- $Q$ in the X-ray data because these data are more sensitive to the heavier atoms in the structure that typically have small atomic displacement parameters whereas the neutron data are dominated by the more disordered part of the structure. (b) X-ray (upper curve) and neutron (lower curve) PDFs. Note the strong contrast in the patterns with (e.g.) a strong O-D peak at $\sim 1 \AA$ in the neutron data that is absent in the X-ray PDF. Other significant differences at low- $r$ are marked by vertical lines. (Using data reported in [143].)

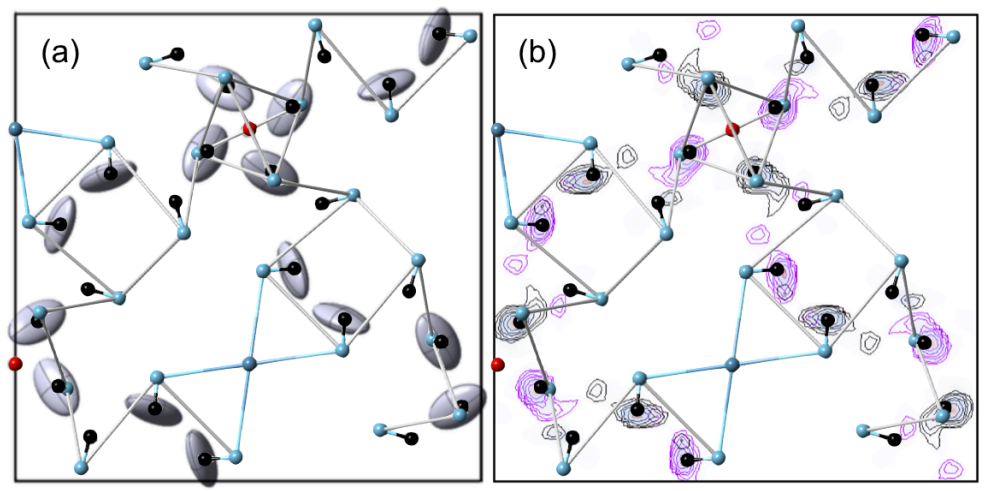

Figure 13. Plots of a (001) section of the unit cell of hydrogrossular at $120 \mathrm{~K}$. (a) Anisotropic atomic displacements parameters from the Rietveld-refined model for D atoms with $z$-values between 0.3 and 0.45 . Ellipses are drawn at $98 \%$ probability. (b) Contour plot of D-atom density within the unit cell from RMCProfile-refined supercell models. Purple and black contours correspond to slices centred on $z \sim 0.35$ and $z \sim 0.39$, respectively, chosen to replicate the D-atom positions in (a) although some density from other D-atoms is visible within this slice. Contours are plotted at approximately 1.6, 3.1, 6.3, 12.5, 25 and 50\% of the maximum intensity. Superimposed on both plots are the Rietveld refined positions of calcium (grey) and oxygen (blue) atoms (with the deuterium (black) atoms connected to these oxygens), and the centres of the $\mathrm{O}_{4} \mathrm{D}_{4}$ tetrahedra (red) within this $z$-range. (Reprinted and adapted from [143] with permission. Copyright 2018 Springer Nature.) 


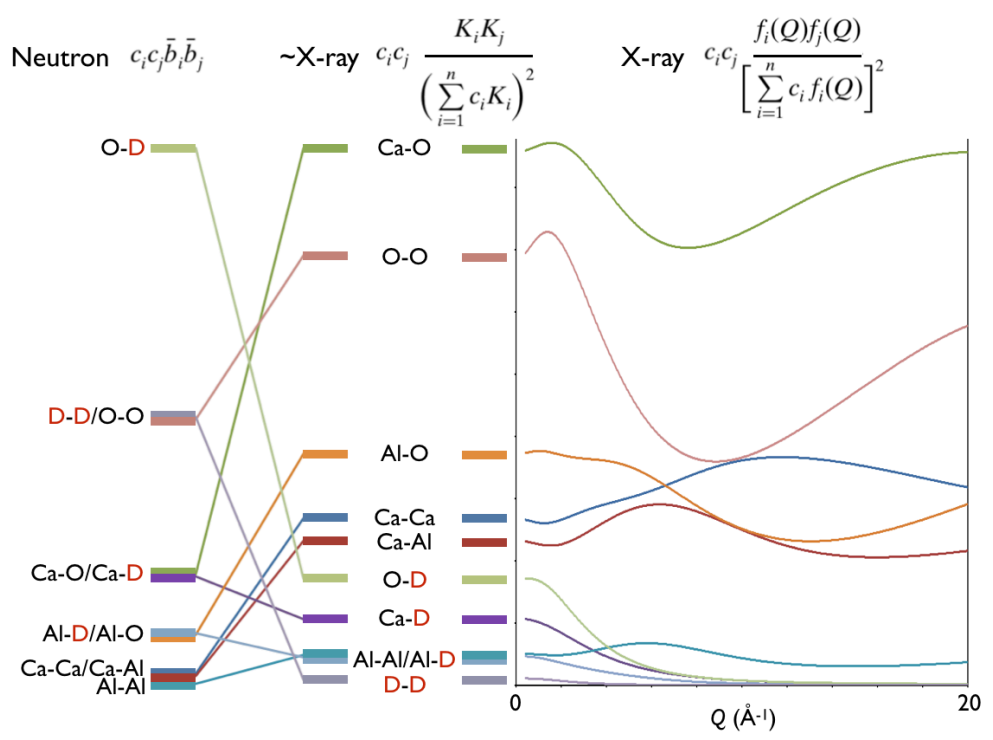

Figure 14. A schematic representation of the different weighting factors for the partial functions in the total scattering for hydrogrossular $\mathrm{Ca}_{3} \mathrm{Al}_{2}\left[(\mathrm{OD})_{4}\right]_{3}$. The left-hand side shows the relative values for neutron total scattering that are dominated by those atom pairs including $\mathrm{D}$ and with high atomic proportions, $c_{i}$. The middle section shows equivalent values for the approximate X-ray weightings to the partial PDFs when bypassing the $Q$-dependence of the atomic scattering factors (see Equation 20); the pairs involving D are now very weakly weighted. The right-hand side shows the correct $Q$-dependent weighting factors for the X-ray structure factor (see Equations 15 and 16) showing that those pairs involving D quickly reduce to zero with increasing $Q$.

were no longer required to do so in the $O$ phase, leading to a significantly different arrangement of $\mathrm{MnO}_{6}$ octahedra where out of plane long $\mathrm{Mn}-\mathrm{O}$ bonds were permitted [146]. Both these pictures are supported by-but not differentiated between - the continued presence of a number of distinct $\mathrm{Mn}-\mathrm{O}$ bond lengths in the neutron PDF that remain largely unchanged through $T_{\mathrm{JT}}[119]$.

The purpose of the later total scattering study was to investigate how the structure might accommodate the dynamically fluctuating distorted $\mathrm{MnO}_{6}$ octahedra within the high-temperature $O$ phase and hence to confirm the detail of the order-disorder transition [144]. A key observation was that there were discontinuous changes in the intensities of the PDFs, particularly for distances between $\sim 3.5 \AA$ and $\sim 6 \AA$ at $T_{\mathrm{JT}}$. They were more prominent in the X-ray PDFs and were a clear indication that the local structure, at distances just beyond the individual polyhedral units, changes across $T_{\mathrm{JT}}$ (Figure 15). This observation prompted RMCProfile refinements of the data which, somewhat surprisingly, produced some unusual distortions in the $\mathrm{MnO}_{6}$ octahedra with the long $\mathrm{Mn}-\mathrm{O}$ bonds at $90^{\circ}$ to each other in the $O$ phase. To attempt to validate this observation, and to compare with the other two possible octahedral arrangements (in-plane only long $\mathrm{Mn}-\mathrm{O}$ bonds at $180^{\circ}$ to each other as in the $O^{\prime}$ phase, and 3SP arrangement of $O^{\prime}$-type distortions arranged in three-dimensions), the authors created small-box approximant structures and refined them using PDFgui. The approximant structures were constructed to replicate the local distortions of the three models below $6 \AA$ and each with the same number of refinable parameters. The goodness-of-fit parameters gave no clear indication as to which model fitted the data better although there was a very marginal preference for the 3SP arrangement.

The reasons for highlighting these two examples in this section are two-fold. First, combining neutron and X-ray total scattering data will often provide a greater understanding of the local structural detail and a greater confidence in the final refined 


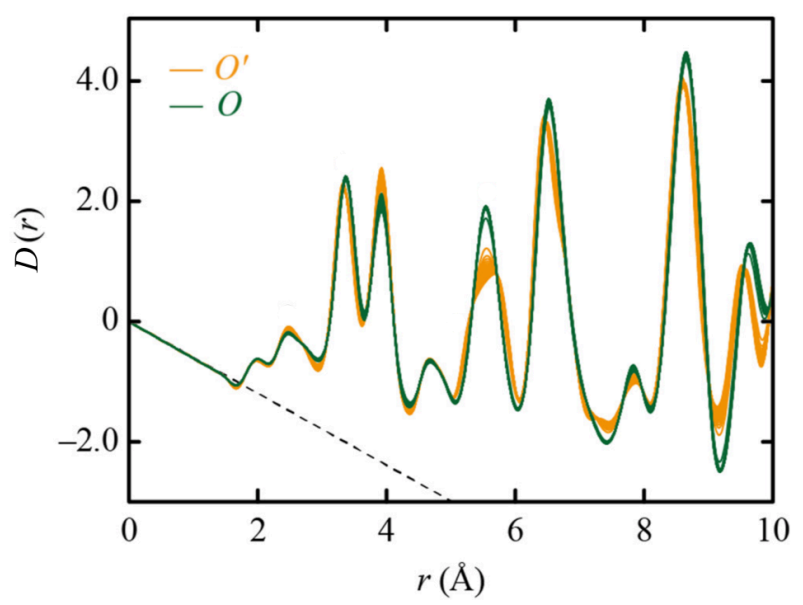

Figure 15. X-ray PDFs from $\mathrm{LaMnO}_{3}$ measured in $10 \mathrm{~K}$ steps between $523 \mathrm{~K}$ and $973 \mathrm{~K}$. Yellow and green lines correspond to data within the $\mathrm{O}^{\prime}$ and $\mathrm{O}$-phases, respectively. There is a clear discontinuous change across the transition in many of the PDF peaks shown; one notable exception being that corresponding to the Mn-O bonds at $\simeq 2 \AA$ which was analysed in the previous neutron work [120]. (Reprinted figure with permission from [144]. Copyright 2017 by the American Physical Society.)

structural models. And secondly, as highlighted in the concluding paragraphs of [144], the refined models should only be presented as definitive after a careful critique. RMCProfile models will always be the most disordered description of a structure consistent with the data and restraints and are unlikely to produce equally plausible, but more ordered, arrangements even if they would give a similarly acceptable fit to the data. Different small-box models can also produce quantitatively similar fits to the PDF and should also be tested for uniqueness. These problems are accentuated at high temperatures and for structures with greater complexity where the information content of the PDFs is reduced and the number of parameters is greater, respectively. Hence the increasing emphasis on including more data and restraints within RMCProfile refinements (see e.g. [147]) and the development of total scattering methods using single crystals (see Section 5.3 below).

\section{A Few Recent Examples}

\subsection{Phase transitions}

\subsubsection{Perovskites}

The work on the different phases of silica, such as the RMCProfile studies of quartz described in Section 3.4.1 above, highlighted the benefits of using PDF methods for studying the way that the local structure changes across phase transitions. Total scattering has been used to good effect for a number of perovskite materials, including $\mathrm{BaTiO}_{3}$ [148], $\mathrm{Ba}_{1-x} \mathrm{Sr}_{x} \mathrm{TiO}_{3}$ [149], PZT [150], NBT [151], PMN [147] and $\mathrm{LaMnO}_{3}$ described above (Section 3.6.4), amongst others.

$\mathrm{BaTiO}_{3}$ undergoes a series of phase transitions from rhombohedral to orthorhombic, tetragonal and then cubic on heating. Single crystal X-ray diffuse scattering from $\mathrm{KNbO}_{3}$, which has an identical series of phase transitions to $\mathrm{BaTiO}_{3}$, was interpreted in terms of the offset of the Ti atom from the centre of its surrounding oxygen octahedron [152]. The Ti atoms at all temperatures are displaced along $\langle 111\rangle$ directions 


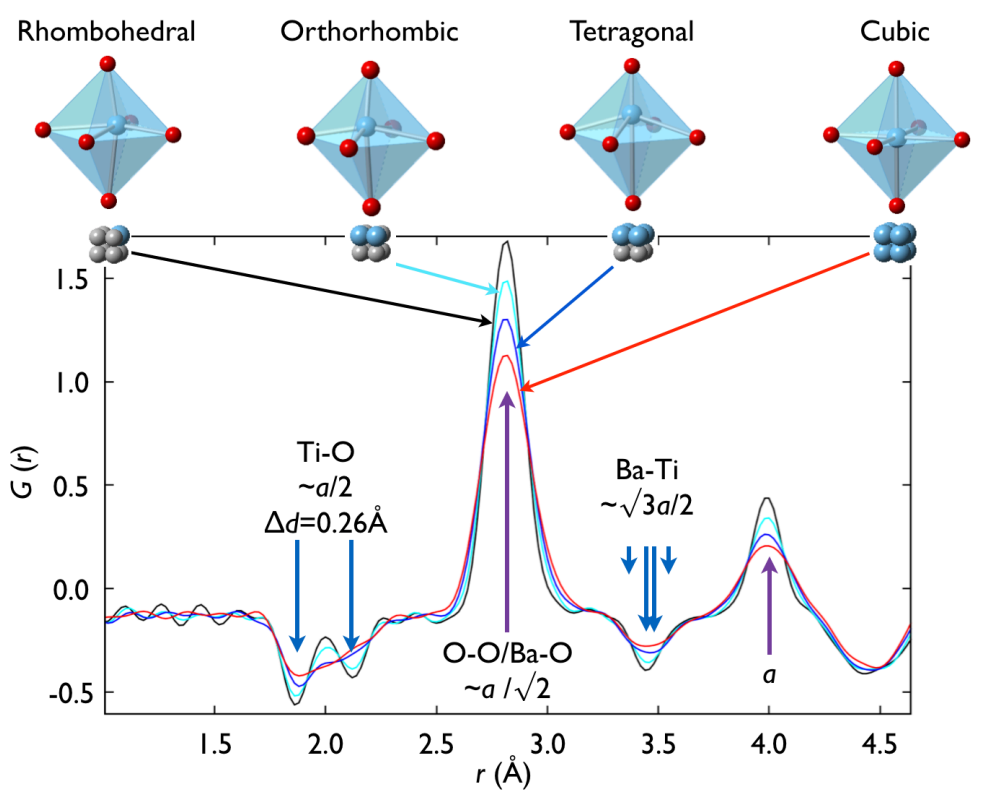

Figure 16. Neutron $\mathrm{PDF}$ data from $\mathrm{BaTiO}_{3}$ within each of its four phases. The Ti-O distance within the $\mathrm{TiO}_{6}$ is split into short- and long-bonds showing that the $\mathrm{Ti}$ is offset along $\langle 111\rangle$ for all temperatures. The blue polyhedra at the top show the average position of the Ti atom for each phase and the sequence of blue occupied spheres below shows how the average position is obtained from different combinations of subsets of the available $\langle 111\rangle$-directions. $a$ refers to the cubic perovskite unit cell parameter. (Using data from [148].)

and the sequence of phase transitions on heating can be understood by this distortion being along one, two, four and eight of the possible $\langle 111\rangle$ directions, arranged to give an apparent $\mathrm{Ti}$ displacement (and macroscopic polarisation direction) along (111), (110), (001) and (000) in the average structure, respectively. Neutron PDFs and RMCProfile analysis supported this picture, the $\mathrm{TiO}_{6}$ octahedra remained distorted with three long and three short $\mathrm{Ti}-\mathrm{O}$ bonds at all temperatures (Figure 16)[148] and RMCProfile models showed that these distortions averaged to (e.g.) provide a net [001] displacement in the tetragonal room temperature phase composed of four predominant $\langle 111\rangle \mathrm{Ti}$ offsets all with a positive $z$ component [149].

In addition the planes of diffuse scattering in [152] were linked to the way these $\langle 111\rangle$ Ti displacements become increasingly uncorrelated along specific $\langle 100\rangle$ directions. At low temperatures the displacements are all correlated and no diffuse scattering is observed. On heating the displacements along $\langle 100\rangle$ 'chains' become uncorrelated in one, two and three dimensions, leading to planes of diffuse scattering in $\{100\}$ planes in reciprocal space in one, two and three directions. This sequence was confirmed using Monte Carlo simulations based only on near-neighbour interactions and local distortions obtained from symmetry-adapted distortion modes refined to the shortrange part of the neutron PDF using constraint tools within PDFgui [148].

Several of the other RMCProfile investigations have determined the rotation of the local polarisation vector as a function of temperature/phase. One good example of this is in the study of $\mathrm{Na}_{0.5} \mathrm{Bi}_{0.5} \mathrm{TiO}_{3}$ (NBT) [151]. At low temperatures within the rhombohedral phase the A-site bismuth ion is displaced predominantly along the pseudo-cubic $\langle 111\rangle$ directions with respect to the centre of the twelve surrounding oxygen anions. On heating, whilst still within this phase, these displacements rotate away from $\langle 111\rangle$ in two opposite directions around a monoclinic plane before settling on a $\langle 001\rangle$ direction within the high-temperature tetragonal phase (see Figure 17). A similar RMCProfile 


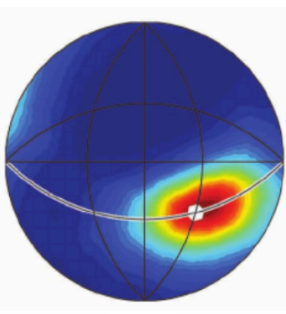

$10 \mathrm{~K}$

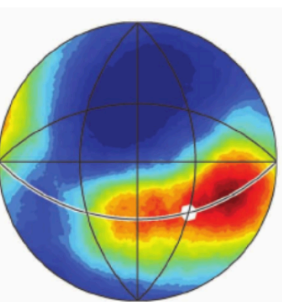

$298 \mathrm{~K}$

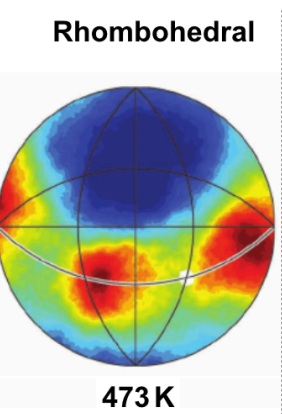

Tetragonal

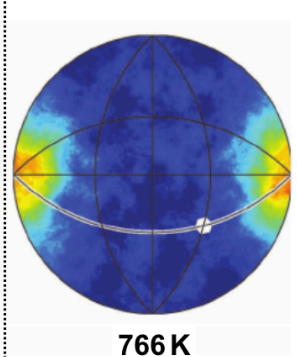

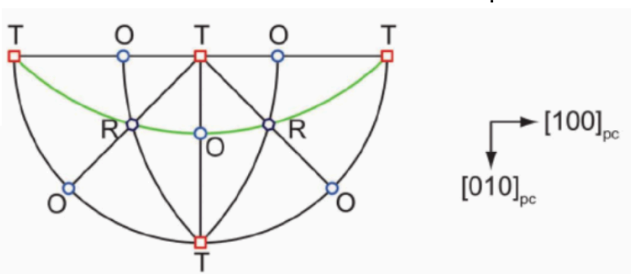

Figure 17. Stereographic projections of bismuth displacement directions from the centroid of their coordination polyhedra of oxygen atoms in $\mathrm{Na}_{0.5} \mathrm{Bi}_{0.5} \mathrm{TiO}_{3}$ at a number of temperatures (upper plots). The lower diagram identifies key positions in the stereographic projections in the upper plots with respect to the perovskite psuedo-cubic (pc) structure: T (tetragonal), O (orthorhombic) and R (rhombohedral) displacements along $\langle 100\rangle,\langle 110\rangle$ and $\langle 111\rangle$ directions, respectively. The green arc identifies one possible set of monoclinic displacements, along $\{110\}$ planes. At low temperature the bismuth atoms are displaced predominantly along the rhombohedal polar direction (identified with a white hexagon) and on heating, whilst still within the rhombohedral phase, the displacements are seen to rotate away in two directions around the highlighted monoclinic plane before localising along a tetragonal direction above the phase transition. (Adapted from [151] with permission. Copyright 2013 WILEY-VCH Verlag GmbH \& Co. KGaA, Weinheim)

analysis of room temperature total scattering data from $\mathrm{PbZr}_{1-x} \mathrm{Ti}_{x} \mathrm{O}_{3}$ (PZT) [150] for $x$-values within the Zr-rich region below the morphotropic phase boundary also provided evidence for local $\mathrm{Pb}$ displacements within the rhombohedral phase reminiscent of two monoclinic phases. These phases are termed $\mathrm{M}_{\mathrm{A}}$ or $\mathrm{M}_{\mathrm{B}}$, depending on whether the displacements were moving away from the rhombohedral directions around $\{110\}$ pseudocubic mirror planes towards tetragonal or orthorhombic directions, respectively. This insight allowed the authors to re-examine their high-resolution powder diffraction data in terms of these monoclinic $\mathrm{M}_{\mathrm{A}}$ and $\mathrm{M}_{\mathrm{B}}$ phases, showing that the inconsistencies in previous structural analyses were most likely related to samples with different $M_{A}$ and $\mathrm{M}_{\mathrm{B}}$ structured domain sizes within the rhombohedral phase. They then used this to provide a credible explanation for the structural confusion around the morphotropic phase boundary.

\subsubsection{Solid state amorphization}

The fact that the PDF can be used irrespective of the nature of the material being measured, whether it is crystalline, amorphous or liquid (or indeed sits anywhere along this spectrum), makes it an ideal tool to investigate transitions between these three states of matter. Note that the study of "pre"-melting in $\mathrm{AgBr}$ described above included measurements of the liquid PDF just above $T_{\mathrm{M}}[50,92]$ (Section 3.4.1). Neutron PDFs were also used to compare the local structures of crystalline silica phases with that of silica glass [153] showing that the glassy structure was remarkably similar to the (instantaneous) local structural arrangements of high-temperature dynamicallydisordered phases of cristobalite and tridymite. 
Although the above silica example is not of a direct transition between a crystalline and amorphous phase it did establish the principle that the PDF was well-placed to compare the structures of such phases. Later work on high-pressure amorphization of the negative thermal expansion (NTE) material $\mathrm{ZrW}_{2} \mathrm{O}_{8}$ used total scattering data measured in-situ and on recovered amorphous samples to investigate the nature of the high-pressure amorphous phase [154,155]. RMCProfile refinement to X-ray and neutron total scattering from a recovered sample was used to test how the crystal structure might change into the amorphous structure.

A good fit to the total scattering data for the amorphous material was obtained for a structure based on a mechanism whereby the pairs of $\mathrm{WO}_{4}$ tetrahedra in the crystalline phase link to form a composite $\mathrm{W}_{2} \mathrm{O}_{8}$ unit (c.f. inset to Figure $9 \mathrm{~b}$; oxygen atom $\mathrm{O} 3$ bonds with neighbouring tungsten W1) with the remaining non-bonded oxygen in this unit (i.e. O4) randomly choosing to bond with the opposite end (i.e. with W2) of one of the three now nearest $\mathrm{W}_{2} \mathrm{O}_{8}$ units to provide a structure with five-coordinated tungsten atoms and with the zirconium atoms remaining six coordinated. It is the 'ice-rules'-like way that the additional W2-O4 bonds form that introduces the disorder necessary to convert the crystal structure into an amorphous one-amorphous from the point of view of the diffraction data at least - with the zirconium atoms largely remaining in their original periodic locations. The additional bonding between $\mathrm{WO}_{4}$ tetrahedra and subsequent relaxation without any bond breaking or significant strain in the structure explains why the structure was recoverable to ambient pressure and the large $\mathrm{WO}_{4}$ motion is consistent with what is known about the dynamics of this NTE material. The remanent crystalline order of the $\mathrm{Zr}$ atoms was not sufficiently coherent to give rise to any Bragg peaks, although it was noted that the contribution from $\mathrm{Zr}-\mathrm{Zr}$ correlations to both the neutron and X-ray data was relatively weak and this might explain why the RMCProfile method did not require the $\mathrm{Zr}$ atoms to move from their original positions significantly. In addition, X-ray total scattering measurements made at pressures up to $10 \mathrm{GPa}$ from a sample in a diamond anvil cell were consistent with this amorphous structural model with no indications of further changes to cation coordinations; the glass structure undergoes simple compression [155].

A similar investigation was performed on the metal organic framework ZIF-4 at high temperature [156]. ZIF-4 possesses $\mathrm{Zn}$ ions that are tetrahedrally coordinated to $\mathrm{N}$ atoms from four different imidazolate ( $\mathrm{Im}, \mathrm{C}_{3} \mathrm{H}_{3} \mathrm{~N}_{2}^{-}$) ions to form a continuous corner linked framework. The shape of the imidazolate ion is such that the $\mathrm{Zn}-\mathrm{Im}-\mathrm{Zn}$ 'bond' angle is similar to that frequently observed in $\mathrm{Si}-\mathrm{O}-\mathrm{Si}$ bonding in zeolites and hence the topologies observed in ZIFs (or zeolitic imidazolate frameworks) are often the same as those found in alumino-silicate-based zeolites. ZIF-4 undergoes a crystal to amorphous transition on heating above $\sim 573 \mathrm{~K}$ to an amorphous phase that is recoverable to room temperature. On further heating the amorphous phase undergoes a transition around $673 \mathrm{~K}$ to a dense crystalline phase, ZIF-zni, which is stable until decomposition or melting, the liquid phase being formed when heated under argon [157]. The structure of the amorphous phase was determined using RMCProfile refinement of model structures to neutron and X-ray total scattering data which found that the most likely structure was akin to that of silica glass, i.e. a continuous random network of corner-sharing $\mathrm{Zn}(\mathrm{Im})_{4}$ tetrahedra, rather than any structure based on the neighbouring crystalline phases (Figure 18). This study has led to a significant amount of related work identifying other amorphous phases, including those formed through ball-milling and melt-quenching, for example $[158,159]$. 


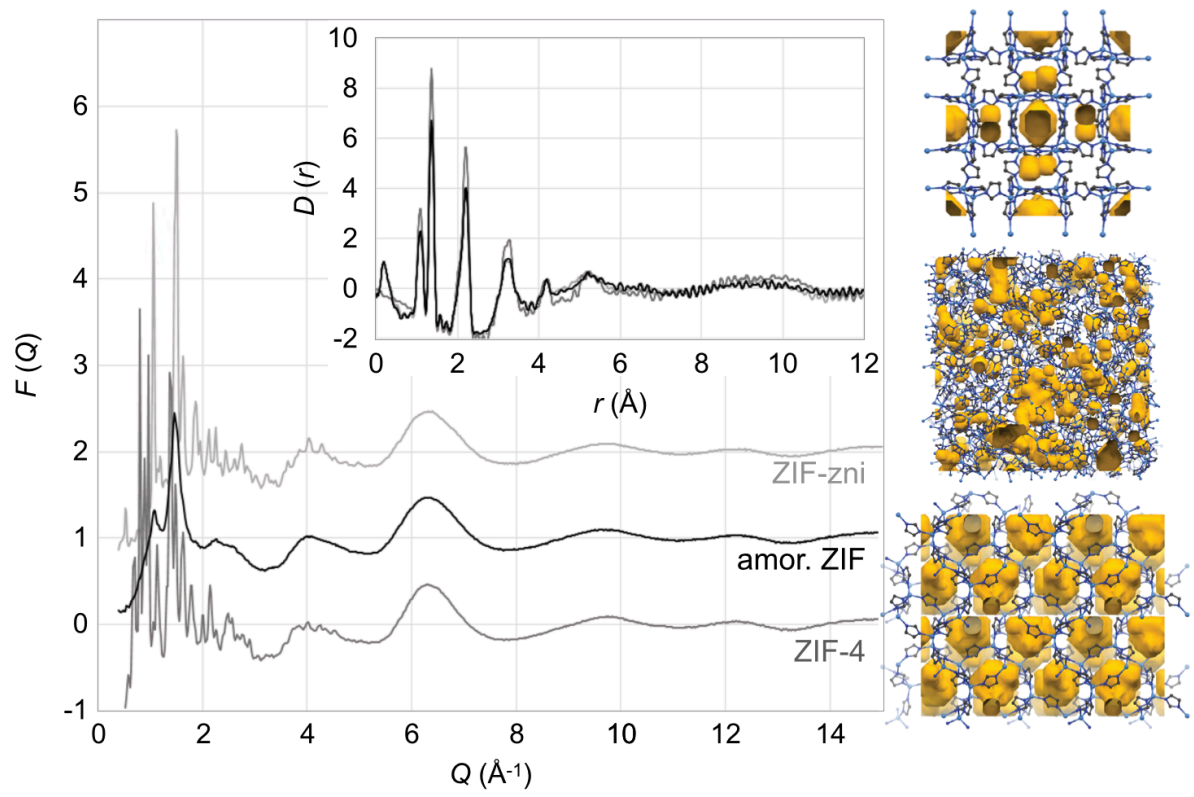

Figure 18. Total scattering from the zeolitic imidazolate framework ZIF-4 on heating. The main figure shows neutron $F(Q)$ data from ZIF-4 on heating through the sequence of phase transitions: ZIF-4 at $478 \mathrm{~K}$, amorphous-ZIF at $573 \mathrm{~K}$ and ZIF-zni at $673 \mathrm{~K}$ (successive plots offset by one vertically). The inset shows the PDFs corresponding to these $F(Q)$ data. The structure plots on the right-hand side correspond to the crystalline structures of ZIF-4 (lower) and ZIF-zni (upper) and the amorphous-ZIF structure (middle) determined from RMCProfile refinement of a silica glass based structure refined to both neutron and X-ray total scattering data. Void volumes are shown in all three structures as yellow isosurfaces. (Adapted from [159] with permission from the PCCP Owner Societies and using data from [156].)

\subsection{Nanoparticles}

\subsubsection{Introductory remarks}

As the name suggests, nanoparticles are materials with a very small particle size (typically less than $100 \mathrm{~nm}$ in all three dimensions). Often they consist of a crystalline core and an outer shell with a different atomic arrangement. Although crystalline, due to their limited size the Bragg peaks from nanocrystals are inherently broad, making routine crystallographic structural analysis difficult. The well-known Scherrer equation[160], $\Delta 2 \theta \simeq \lambda /(D \cos \theta)$, relating the full width half maximum, $\Delta 2 \theta$, of a Bragg peak measured at scattering angle, $2 \theta$, and using radiation of wavelength, $\lambda$, to the size of the crystallite, $D$, shows that as nanoparticles become smaller than $10 \mathrm{~nm}$ the information obtainable from the Bragg peaks is severely hampered by peak overlap and reduced peak heights. However, a PDF from a nanoparticle will still measure the pair distances accurately, irrespective of how small it is, and can therefore be used to determine their structure. Furthermore, the variation of the PDF peak intensities as a function of distance may be used to determine the particle size and - in favourable situations - shape. (Additional general discussions of the PDF and nanoparticle structure are given in two recent reviews. Reference [161] places PDF work in the context of other X-ray scattering techniques and [162] provides a very recent summary of the range of PDF work on nanomaterials, including work carried out in-situ and inoperando.)

Complications in the study of nanoparticles arise because they are often not monodispersed (i.e. a sample of nanoparticles usually contains particles of many different 
sizes), they are usually coated with additional material to prevent them agglomerating and they might have to remain in solution for scattering measurements. The latter two complications means that poor scattering contrast may be an issue, especially for the smallest nanoparticles where the number of atoms in the outer coating may be greater than those in the nanoparticle itself. This is more problematic for neutron total scattering than from X-ray since the coating is often composed of organic molecules which would easily dominate the nanoparticle signal in a neutron measurement. As a result, PDF experiments on nanoparticles were, at least initially, dominated by X-ray measurements on nanocrystals containing heavier elements (e.g. gold, platinum etc.).

Neder and co-workers have published a series of papers on the methodologies for analysing PDF data from nanocrystals (e.g. [163-166]). Most of the complications in the interpretation arise from the granular nature of the samples outlined in the paragraph above. Atom pairs tend to be strongly correlated within the core of the nanoparticle, correlated within the nanoparticle (core and surface atoms), only weakly correlated to the coating and uncorrelated to the surrounding nanoparticles. It is the calculation of the 'background' contribution to the PDF from these uncorrelated atoms that is tricky, being dependent on nanoparticle distributions of size and shape within the sample. In some senses this 'background' of correlations can be considered as an envelope function, appearing to suppress the PDF peak intensities that would arise from a long-ranged periodic sample with the same structure as that of the nanoparticle. This is the approach taken by PDFgui [55] using envelope functions calculated for simple nanoparticle shapes such as spheres. A more complete methodology, whereby nanoparticles are 'built' using DISCUS [167] and DIFFEV [168] programs is more flexible, allowing the nanoparticles to contain defects, distinct surface structure and coatings, is described in [166]. Similarly detailed nanocrystals can be built and refined using the Debye equation explicitly, although this approach is not taken further here; instead the reader is referred to work using the DEBUSSY program, see for example [169]

\subsection{2. 'Magic-sized' gold metal clusters}

One model-building nanoparticle study investigated the structure of the so-called 'magic-sized' gold metal clusters [170]. The authors investigated $\mathrm{Au}_{144}(\mathrm{SR})_{60}$ where 'SR' were a number of different thiolate ligands. The models were mostly based on various already-proposed structures, composed of a crystalline gold core covered in a shell of additional gold atoms (to form a 'grand core') followed by a further covering of gold and sulphur atoms that form -SR-Au-SR- 'staples' over the surface (see, for example, Figure 19). The nanoparticle is therefore very small with the grand gold core below the staples layer consisting of only three shells of atoms with a diameter below $20 \AA[171]$ and the Bragg peaks in the X-ray diffraction pattern are very broad. The models were refined to X-ray PDF data showing that some samples had truncated decahedron structures, others had the previous-proposed icosahedral structure (based on, for example, DFT calculations [171] and STM measurements [172]) whereas in other samples the two structures coexisted.

\subsubsection{Titania nanorods}

$\mathrm{TiO}_{2}$ nanoparticles have attracted considerable attention because of the ability to tune their optical properties through varying their size and shape. The thermodynamically stable form of bulk titania has the rutile structure, whereas for nanoparticles 


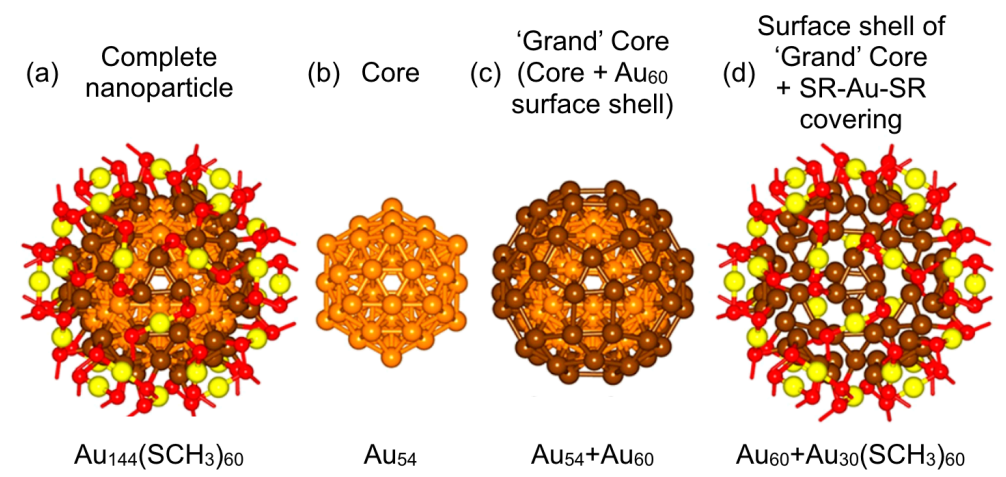

Figure 19. A structure of $\mathrm{Au}_{144}(\mathrm{SR})_{60}$ with $\mathrm{R}=\mathrm{CH}_{3}$. The gold atoms are shown as orange, brown or yellow spheres, depending on where they are in the structure with $\mathrm{S}$ atoms as red spheres and methyl groups (not shown) attached to the end of the red sticks. (Reprinted with permission from [172]. Copyright 2013 American Chemical Society.)

the less-common anatase and brookite structures are preferred [173]. In a recent study Krayzman and colleagues used X-ray total scattering and reverse Monte Carlo refinements to try to determine the structure of nanorods of $\mathrm{TiO}_{2}$ [174]. Samples of the nanorods with two different lengths were prepared mostly with the brookite structure, although it was found that some included intergrowths with the anatase structure and the samples also contained irregularly-shaped anatase-structured nanoparticles. STEM measurements confirmed that on average the nanorods were either $\sim 15 \mathrm{~nm}$ long and $\sim 3 \mathrm{~nm}$ diameter or $\sim 25 \mathrm{~nm}$ long and $\sim 4 \mathrm{~nm}$ diameter (depending on the sample) with both samples having the long nanorod direction aligned along the [001] brookite axis and clear $\{210\}$ facets on the side of the nanorods.

Model structures, to be refined against the total scattering data, were constructed by forming suitably-sized and faceted nanorods of the known brookite structure and additional cuboid-shaped nanoparticles with the anatase structure. The refinements followed the usual RMCProfile protocols (see Section 3.4.1), albeit with each atomic configuration consisting of a single nanoparticle surrounded by empty space to override the periodic boundary conditions imposed on RMCProfile models. Use was also made of the latest RMCProfile codes that improve the speed, and hence increase the size of the models that are tractable within a practical timescale $[147,175,176]$. The refined model structures were then used to investigate changes to cell parameters relative to the bulk values and how the atomic environment might vary across the nanorods. It was found that there was an overall expansion of the brookite lattice and that the $\mathrm{Ti}$ displacements and $\mathrm{TiO}_{6}$ octahedral distortions increase going radially from the centre to the edges of the nanorod. Despite these changes, the atomic arrangements retained the overall expected symmetry, a result supported by their accompanying DFT calculations.

\subsubsection{Impact of low-Q scattering}

Another way to visualise the nanoparticle sample's granularity is to take clues from the analysis of small-angle scattering. The influence of this scattering on the PDF was considered in a study of amorphous germanium samples that contained voids [177] using theory developed a couple of years earlier [178]. The theory, as applied specifically to nanoparticles, was later developed more completely [179]. The information from small-angle scattering was incorporated into the example in the previous section (Sec- 
(a)

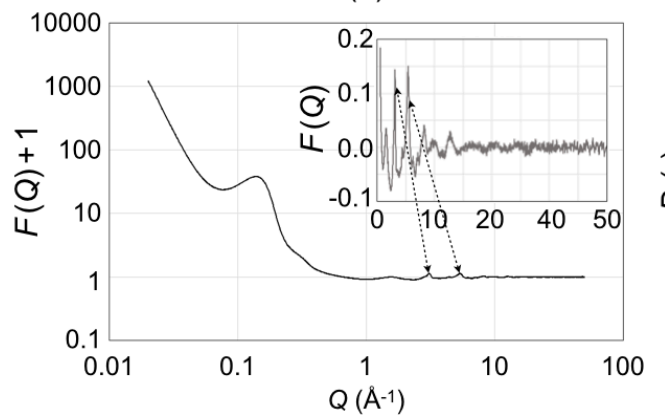

(b)

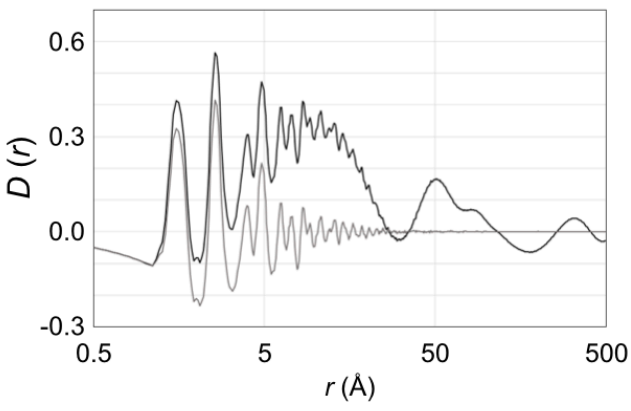

Figure 20. Neutron total scattering measured on the Nimrod diffractometer at ISIS from a hexagonal mesoporous silica with a wormhole framework structure (HMS-wormhole). Despite the name, samples of HMSwormhole usually only have very small regions, if any, with hexagonal wormhole order, and the particle sizes are often less than $100 \mathrm{~nm}$ [181]. The main panel of (a) shows the total scattering structure factor-including substantial scattering at low- $Q$ - on a log-log plot (with $F(Q)$ offset by one to facilitate the log-y-axis); the inset shows $F(Q)$ in its more usual presentation and over a typical $Q$-range for PDF measurements. (b) shows the PDFs from the data in (a) (black line) and from the more limited $Q$-range given in (a) inset (grey line) (plotted against $r$ on a log-scale). Notice that although the high-frequency components ( $\mathrm{Si}-\mathrm{O}, \mathrm{O}-\mathrm{O}$ distances etc.) are present in both PDFs, the PDF from the full range of $Q$ reveals large underlying low-frequency oscillations from the mesoporous structure, which for this sample has a characteristic distance of around $40 \AA$. (Using data from [180].)

tion 4.2.3) where the it was used to determine the (distributions of) size and shape of the nanoparticles; information that is also possible to obtain from scanning tunnelling microscopy (STM). A nanoparticle sample will scatter into the "small angle" (i.e. very low- $Q$ ) region due to density variations between the nanoparticles and surrounding material. The total scattering signal from the sample includes this scattering, but on many PDF instruments it is not measured. The Fourier transform of this total scattering (i.e. Bragg plus diffuse plus low- $Q$ scattering) will provide a pair distribution function composed of the familiar PDF pattern on a low-frequency oscillatory signal arising from the low- $Q$ scattering (see examples from mesostructured silicas in [180], and Figure 20). By truncating the total scattering to a minimum $Q$-value that is above the low- $Q$ scattering this oscillatory signal is removed and the PDF instead sits on a flat 'background' that is unrepresentative of the overall sample signal.

\section{Perspectives and future opportunities}

I hope that this review article has successfully demonstrated that the PDF method is now fully established within the suite of crystallographic techniques available for revealing crystal structure. Furthermore, it fulfils a unique role in being able to explore the way that crystal structures disorder at the local level through a relatively simple experiment and careful analysis. There are inevitably gaps in the review-for example I have not compared in detail the relative benefits of PDF experiments with XAFS or single crystal diffuse scattering experiments, or analyses of total scattering using the Debye equation - but I have tried to be as all encompassing within the PDF field as possible, albeit with a greater emphasis on 'big box' methods which have tended to be under-represented in the existing review literature.

The PDF method has been reappropriated from the liquid and amorphous structure community and applied to a wide range of crystalline structure problems, made possible through the improved instrumentation and analysis methods developed in 
the past thirty-odd years. With the rapid developments in the method in these thirty years, perhaps it would be pertinent to consider what might be around the corner and available thirty years from now? I therefore conclude this review with some brief musings about the future - future instrumentation, analysis software and directions.

\subsection{Future instrumentation}

As already discussed in Section 3.1 the PDF method within crystallography was transformed through dedicated time-of-flight neutron and synchrotron X-ray total scattering diffractometers giving access to high- $Q$ data and rapid data collections. It is now far more common for central facility developers to include these instruments within the overall suite of new instruments as a priority and I expect this to continue. Access to PDF instrumentation will therefore grow, with greater capacity and instruments spread around the world more widely. I anticipate that most, if not all, new X-ray synchrotrons with high energy X-ray beams and so-called 'short-pulse' spallation neutron sources will have at least one PDF instrument.

I also believe that PDF experiments will soon become possible at X-ray free-electron lasers (XFELs). There will be challenges with this, mostly in terms of experimental stability, but XFELs are pushing to higher X-ray energies $(20-25 \mathrm{keV}$ are possible now; still higher energies might be available using the harmonics of the XFEL beam) and with this - and their intense sharply pulsed X-ray beams - there will be good opportunities for time-resolved studies of disordering structural processes. Such studies might investigate the mechanisms of phase transitions, the rapid structural response to extreme heating, the PDFs of warm dense matter or the way that structure changes under laser irradiation (essentially pump-probe measurements with the X-ray PDF as the probe). XFELs will enable unique, potentially ground-breaking, PDF experiments albeit with a limited experimental throughput.

\subsection{Future software}

The second driver of the growth of total scattering within crystallography was the development of software and in particular the programs RMCProfile [51] and PDFgui [55]. These, and the programs that stemmed from them, gave crystallographers the tools to interpret their PDF data in a way to provide clear physical insight. PDFgui is a tool that is conceptually very simple - a 'real-space Rietveld' method - that provides a smooth access point for those who are familiar with the regular Rietveld method for refining crystal structures. RMCProfile is more demanding in terms of the data and the time and effort required to obtain good results, but is more generally applicable. Increasingly general Rietveld refinement codes will offer PDF fitting options, indeed they are already available within the TOPAS software [182] (see [141] where TOPAS was used for Rietveld refinement of the Bragg powder pattern and for small-box modelling of the PDF) and may soon be incorporated within GSAS-II [183], the modern incarnation of the original GSAS-EXPGUI $[98,99]$ version of Rietveld refinement software; RMCProfile has very recently been made available from within GSAS-II [184]. The RMCProfile code has also recently been optimised for speed in order to increase the size of models that can be refined [175] as has the related EPSR code for liquid and amorphous materials [44]. Its scope has also been broadened to enable refinement to a greater range of structural data (e.g. $[147,185]$ ), including single crystal diffuse scattering [147]. I expect to soon see RMCProfile being more widely used for nanopar- 
ticle studies and for analysis of mixed phase samples as well as developing ways to make the program more straightforward to use.

\subsection{Future directions}

Total scattering/PDF methods continue to play an important role in the structural understanding of new materials. Taking some examples from those materials that have come to prominence in the last few years, relevant PDF studies have been conducted on perovskite photovoltaics [186,187], possible lithium battery anodes (e.g. [188]), local orbital effects in iridium-containing materials [189] and local nematicity in iron-based superconductors [190]; other examples, and examples from materials discovered less recently have been described earlier in this article. With the ever-increasing chemical, structural and magnetic complexity of new materials the need for quantitative measurements of local structure will only increase [191]. An example of this within chemistry might be a role in the growing need to understand defect structures within metal-organic frameworks (e.g. [192,193]). Furthermore, improvements in speeds of data collection and in atomistic modelling, especially towards larger-sized multi-phase modelling, which are currently under development, will lead to more detailed structural investigations of chemical processes such as those promoted by catalysts and the nucleation and growth of nanoparticles. Within physics, a good recent example might be the use of total magnetic scattering to elucidate complex disordered magnetic structures such as the 10-spin multipole loops within the spin-liquid gadolinium gallium garnet [194]. In contrast, the application of PDF methods within protein crystallography has been very limited, although there is an increasing interest in measuring the total scattering from single crystal protein samples to extract the diffuse scattering for further analysis (e.g. $[195,196])$.

Indeed it is in the area of single crystal total scattering where one of the recent interesting developments have been made, using the so-called 3D- $\triangle \mathrm{PDF}$ approach $[197,198]$. The idea is that the Fourier transform of the total scattering from a single crystal sample will be a three-dimensional pair distribution function (3D-PDF). The 3D-PDF function may be split into two terms, the Fourier transform of the Bragg peaks (which is the familiar Patterson function [17] giving the relative positions of atoms within the average structure) and the $3 \mathrm{D}-\triangle \mathrm{PDF}$, which captures the deviations of atom pairs from those found in the Patterson function. As long as the diffuse scattering can be accurately separated from the Bragg scattering, an experimental $3 \mathrm{D}-\triangle \mathrm{PDF}$ can be determined directly as the Fourier transform of the measured single crystal diffuse scattering. The two clear advantages of this approach over the (powder) PDF method are that the $3 \mathrm{D}-\triangle \mathrm{PDF}$ includes directional information and that the contribution to the $3 \mathrm{D}-\mathrm{PDF}$ which is not associated with the average structure is more visible. The disadvantages are that it is more difficult to collect a full three-dimensional set of total scattering data from a single crystal, although this is significantly more straightforward at X-ray synchrotrons than it used to be, and the reciprocal space X-ray data typically extend to a smaller maximum $|\mathbf{Q}|$ than the value of $Q_{\text {max }}$ routinely achieved for a PDF measurement from a powder (but see the possibilities with time-of-flight neutron instruments [199]). The restricted real-space resolution determined by the reduced maximum scattering vectors is mitigated for by calculating the $3 \mathrm{D}-\triangle \mathrm{PDF}$ directly from the diffuse scattering; the strong - and broadened - average structural features that would be present in the 3D-PDF are experimentally removed. The program YELL [198] can be used to refine structures to the 3D- $\triangle \mathrm{PDF}$ and has 
been used to good effect in a number of cases, including in a wide-ranging study of the vacancy arrangements in Prussian blue analogues [200]. 3D- $\triangle \mathrm{PDF}$ concepts have also been extended to magnetic systems, i.e. $3 \mathrm{D}-\mathrm{m} \Delta \mathrm{PDF}$, and applied to measurements of magnetic diffuse scattering from single crystal samples [201].

It is appropriate that this review ends with this discussion of three-dimensional PDFs; the link between powder total scattering and the Patterson function was first made in 1934 by Warren, Gingrich and Patterson $[17,18]$ at the birth of the two techniques. The intervening $80+$ years have brought the experimental and computational methods to a sufficiently advanced state that total scattering from single crystals can now be usefully incorporated within a Patterson function, further strengthening the conceptual link identified all those years ago.

\section{Acknowledgements}

I am grateful to the many people who I have worked with on total scattering problems; their help, advice and support are the backdrop to this review. More specifically I thank Andrew Goodwin, Daniel Bowron and Stephen Hull who kindly read through and commented on the manuscript in advance of submission.

\section{Notes on contributor}

David Keen began applying neutron total scattering to crystalline structure disorder problems during his DPhil thesis work in Oxford with William Hayes and Robert McGreevy in the 1980s where he worked with Robert to develop the reverse Monte Carlo (RMC) method while it was still in its infancy. He then went to work at the ISIS Neutron Facility, Rutherford Appleton Laboratory, continuing his total scattering research using the GEM powder diffractometer, as well as developing single crystal diffuse scattering methods on the SXD time-of-flight Laue instrument. His early interest was in using their newly established total scattering methods to characterise the disorder associated with ion transport in silver and copper superionic conductors but this quickly broadened to encompass a much wider variety of materials. A collaboration with Martin Dove, initially studying the similarities in polyhedral disorder in silica poymorphs, soon diversified to other related areas including, amongst others, negative thermal expansion materials. Their collaboration-together with Matt Tucker and Andrew Goodwin-resulted in the RMCProfile refinement code and led to further developments of 'big-box' methods for analysing magnetic total scattering.

More recently he has been incorporating X-ray total scattering measurements in his work. Although one of his first RMC papers [202] used neutron and X-ray total scattering to model silica glass structure, it was not until his early forties that he first filled an X-ray-sized glass capillary, so you might say that he has been a somewhat late adopter of X-rays! His particular interests here are in optimising the experimental method to obtain the best quality X-ray total scattering data and in demonstrating the benefits of combining neutron and X-ray PDF data when refining structural models.

He has been President of the British Crystallographic Association, the Chair of the International Advisory Committee for all the three-yearly Budapest RMC Conferences and an advisor for several total scattering instrument designs at central facilities around the world. 


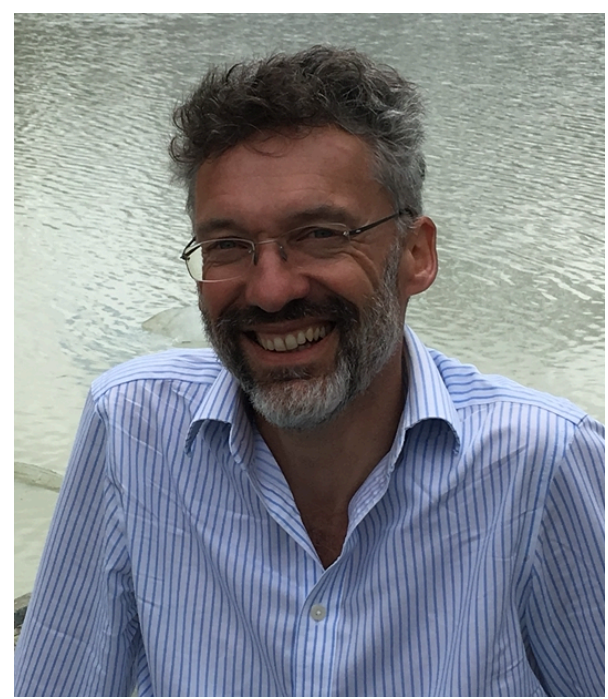

Figure 21.

\section{References}

[1] Friedrich W., Knipping P. and Laue M. Interferenz-Erscheinungen bei Röntgenstrahlen Sitzungsber. Kgl. Bayer: Akad. Wiss. 1912, 303-322

[2] Friedrich W. Eine neue Interferenzerscheinung bei Röntgenstrahlen. Physikalische Zeitschrift 1913, 14, 317-319

[3] Debye P. Zerstreuung von Röntgenstrahlen. Nachrichten von der Gesellschaft der Wissenschaften zu Göttingen, Mathematisch-Physikalische Klasse. 1915, 70-76; Annalen der Physik. 1915, 46, 809-823

[4] Ehrenfest P. On interference phenomena to be expected when Röntgen rays pass through a di-atomic gas. Proc. Amsterdam Acad. 1915, 17, 1184-1190

[5] Debye P. and Scherrer P. Interferenzen an regellos orientierten Teilchen im Röntgenlicht. II. Nachrichten von der Gesellschaft der Wissenschaften zu Göttingen, MathematischPhysikalische Klasse 1916, 16-26

[6] Gelisio L and Scardi P. 100 years of Debye's scattering equation. Acta Cryst. A 2016, 72, $608-620$

[7] Debye P. and Scherrer P. Interferenzen an regellos orientierten Teilchen im Röntgenlicht. I. Nachrichten von der Gesellschaft der Wissenschaften zu Göttingen, MathematischPhysikalische Klasse 1916, 1-15

[8] James RW. The optical principles of the diffraction of X-rays. G. Bell and Sons Ltd (London) 1958

[9] Debye P. Note on the scattering of X-rays. J. of Math. and Phys. Camb. Mass. (later incorporated into Studies in Appl. Math.) 1925, 4, 133-147

[10] Zernike F. and Prins JA. Die Beugung von Röntgenstrahlen in Flüssigkeiten als Effekt der Molekülanordnung. Zeit. Physik 1927, 41, 184-194

[11] Debye P. and Menke H. Bestimmung der inneren Struktur von Flüssigkeiten mit Röntgenstrahlen. Physikal. Zeit. 1930, 31, 797; Untersuchung der molekularen Ordnung in Flüssigkeiten mit Röntgenstrahlung Ergebnis. d. techn. Röntgenkunde 1931, 2, 1

[12] Bosio L., Cortes R. and Segaud C. X-ray diffraction study of liquid mercury over temperature range 173 to $473 \mathrm{~K}$. J. Chem. Phys. 1979, 71, 3595-3600

[13] Tarasov LP. and Warren BE. X-ray diffraction study of liquid sodium. J. Chem. Phys. 1936, 4, 236-238

[14] Warren BE. and Gingrich NS. Fourier integral analysis of X-ray powder patterns Phys. 
Rev. 1934, 46, 368-372

[15] Warren BE. X-ray diffraction. (Reprint) Dover Publications Inc. (Mineola, N.Y.) 1990 (From original publication by Addison-Wesley Publ. Co., Inc. (Reading, Mass.) 1969)

[16] Guinier A. X-ray diffraction in crystals, imperfect crystals, and amorphous bodies. (Reprint) Dover Publications Inc. (Mineola, N.Y.) 1994 (from English edition originally published by W. H. Freeman and Co. (San Francisco) 1956)

[17] Patterson AL. A Fourier series method for the determination of the components of interatomic distances in crystals. Phys. Rev. 1934, 46, 372-376

[18] Warren BE. X-ray diffraction study of carbon black. J. Chem. Phys. 1934, 2, 551-555

[19] Hultgren R, Gingrich NS. and Warren BE. The atomic distribution in red and black phosphorus and the crystal structure of black phosphorus. J. Chem. Phys. 1935, 3, 351355

[20] Thomas CD. and Gingrich NS. The atomic distribution in the allotropic forms of phosphorus at different temperatures. J. Chem. Phys. 1938, 6, 659-665

[21] Warren BE. and Biscoe J. The structure of silica glass by X-ray diffraction studies. J. Am. Ceram. Soc. 1938, 21, 49-54

[22] Warren BE., Krutter H. and Morningstar O. Fourier analysis of X-ray patterns of vitreous $\mathrm{SiO}_{2}$ and $\mathrm{B}_{2} \mathrm{O}_{3}$. J. Am. Ceram. Soc. 1936, 19, 202-206

[23] Bernal JD. and Fowler RH. A theory of water and ionic solution, with particular reference to hydrogen and hydroxyl ions. J. Chem. Phys. 1933, 1, 515-548

[24] Franklin RE. The interpretation of diffuse scattering X-ray diagrams of carbon Acta Cryst. 1950, 3, 107-121

[25] Kaplow R, Averbach BL and Strong SL. Pair correlations in solid lead near the melting temperature J. Phys. Chem. Solids 1964, 25, 1195-1204

[26] Fessler RR, Kaplow R and Averbach BL. Pair correlations in liquid and solid aluminium Phys. Rev. 1966, 150, 34-43

[27] Lagneborg R and Kaplow R. Radial distribution functions in solid cobalt Acta Metall. 1967, 15, 13-24

[28] Harris PJF. Rosalind Franklin's work on coal, carbon, and graphite. Interdisciplinary Sci. Rev. 2001, 26, 204-210

[29] Maddox B. Rosalind Franklin the dark lady of DNA. HarperCollins 2002

[30] Ruppersberg H and Seeman HJ. Die Atomverteilungskurven für festes und flüssiges Aluminium nach Untersuchungen an SAP. Zeit. Naturforschg. 1965, 20a, 104-109

[31] Kaplow R, Rowe TA and Averbach BL. Atomic arrangement in vitreous selenium Phys. Rev. 1968, 168, 1068-1079

[32] Rechtin MD, Renninger AL and Averbach BL. Monte Carlo models of amorphous materials J. Non-Cryst. Solids 1974, 15, 74-82

[33] Renninger AL, Rechtin MD and Averbach BL. Monte Carlo models of atomic arrangements in arsenic-selenium glasses J. Non-Cryst. Solids 1974, 16, 1-14

[34] Wollan EO and Shull CG. The diffraction of neutrons by crystalline powders Phys. Rev.. 1948, 73, 830-841

[35] Shull CG, Wollan EO, Morton GA and Davidson WL. Neutron diffraction studies of NaH and NaD Phys. Rev.. 1948, 73, 842-847

[36] Chamberlain O. Neutron diffraction in liquid sulfur, lead and bismuth Phys. Rev.. 1950, 77, 305-313

[37] Furukawa K. The radial distribution curves of liquids by diffraction methods Rep. Prog. Phys. 1962, 25, 395-440

[38] Enderby JE, North DM and Egelstaff PA. The partial structure factors of liquid Cu-Sn Phil. Mag. 1966, 14, 961-970

[39] Bondot P. Essai de séparation des distributions de paires dans $\mathrm{GeO}_{2}$ vitreux á partir de l'effet de diffusion anormale Acta Cryst. A 1974, 30, 470-471

[40] Fischer HE, Barnes AC and Salmon PS. Neutron and X-ray diffraction studies of liquids and glasses Rep. Prog. Phys. 2006, 69, 233-299

[41] Soper AK. Computer simulation as a tool for the interpretation of total scattering data 
from liquids and glasses. Mol. Simul. 2012, 38, 1171-1185

[42] Soper AK. Empirical potential Monte Carlo simulation of fluid structure. Chem. Phys. 1996, 202, 295-306

[43] Soper AK. Joint structure refinement of x-ray and neutron diffraction data on disordered materials: application to liquid water. J. Phys. Condens. Matter 2007, 19, 335206

[44] Youngs TGA. Dissolve: next generation software for the interrogation of total scattering data by empirical potential generation. Mol. Phys. 2019, 117, 3464-3477

[45] Kofalt DD, Nanoa S, Egami T, Wong KM and Poon SJ. Differential anomalous-X-rayscattering studies of icosahedral and amorphous $\mathrm{Pd}_{58.8} \mathrm{U}_{20.6} \mathrm{Si}_{20.6}$ Phys. Rev. Lett. 1986 , $57,114-117$

[46] Kofalt DD, Morrison IA, Egami T, Preische S, Poon SJ and Steinhardt PJ. Quasicrystallinity of icosahedral $\mathrm{Pd}_{58.8} \mathrm{U}_{20.6} \mathrm{Si}_{20.6}$ Phys. Rev. B 1987, 35, 4489-4492

[47] Nanoa S, Dmowski W, Egami T, Richardson, Jr. JW and Jorgensen JD. Structure of Al-Mn-Cr-Si quasicrystals studied by pulsed neutron scattering Phys. Rev. B 1987, 35, $435-440$

[48] McGreevy RL and Pusztai L. Reverse Monte Carlo simulation: A new technique for the determination of disordered structures Mol. Simul. 1988, 1, 359-367

[49] McGreevy RL. Reverse Monte Carlo modelling J. Phys. Condensed Matter 2001, 13, R877-R913

[50] Keen DA, Hayes W and McGreevy RL. Structural disorder in AgBr on the approach to melting J. Phys. Condensed Matter 1990, 2, 2773-2786

[51] Tucker MG, Keen DA, Dove MT, Goodwin AL and Hui Q. RMCProfile: reverse Monte Carlo for polycrystalline materials J. Phys.: Condensed Matter 2007, 19, 335218

[52] Billinge SJL, Davies PK, Egami T and Catlow CRA. Deviations from planarity of copperoxygen sheets in $\mathrm{Ca}_{0.85} \mathrm{Sr}_{0.15} \mathrm{CuO}_{2}$ Phys. Rev. B 1991, 43, 10340-10352

[53] Billinge SJL, Egami T, Richards DR, Hinks DC, Dabrowski B, Jorgenen JD and Volin KJ. Local structural change close to $T_{r m c}$ in in $\mathrm{Nd}_{2-x} \mathrm{Ce}_{x} \mathrm{CuO}_{4-y}$ Physica $\mathrm{C}$ 1991, 179, 279 285

[54] Billinge SJL and Egami T. Short-range atomic structure of $\mathrm{Nd}_{2-x} \mathrm{Ce}_{x} \mathrm{CuO}_{4-y}$ determined by real-space refinement of neutron-powder-diffraction data Phys. Rev. B 1993, 47, 14386-14406

[55] Farrow CL, Juhas P, Liu JW, Bryndin D, Božin ES, Bloch J, Proffen Th and Billinge SJL. PDFfit2 and PDFgui: computer programs for studying nanostructure in crystals J. Phys.: Condensed Matter 2007, 19, 335219

[56] Keen DA. A comparison of various commonly used correlation functions for describing total scattering J. Appl. Cryst. 2001, 34, 172-177

[57] Soper AK and Barney ER. Extracting the pair distribution function from white-beam X-ray total scattering data. J. Appl. Cryst. 2011, 44, 714-726

[58] Peterson PF, Božin ES, Proffen T and Billinge SJL. Improved measures of quality for the atomic pair distribution function. J. Appl. Cryst. 2002, 36, 53-64

[59] Egami T and Billinge SJL. Underneath the Bragg peaks structural analysis of complex materials. Pergamon Materials Series (Series Ed. Cahn RW.) Volume 7, Elsevier Ltd. (Oxford, U.K.) 2003

[60] Olds D, Saunders CN, Peters M, Proffen T, Neuefeind J and Page K. Precise implications for real-space pair distribution function modeling of effects intrinsic to modern time-offlight neutron diffractometers. Acta Cryst. A 2018, 74, 293-307

[61] Fischer HE, Cuello GJ, Palleau P, Feltin D, Barnes AC, Badyal YS and Simonson JM. D4c: A very high precision diffractometer for disordered materials. Appl. Phys. A 2002, 74, s160-s162

[62] Paściak M, Ondrejkovic P, Kulda J, Vaněk P, Drahokoupil J, Steciuk G, Palatinus L, Welberry TR, Fischer HE, Hlinka J and Buixaderas E. Local structure of relaxor ferroelectric $\mathrm{Sr}_{x} \mathrm{Ba}_{1-x} \mathrm{Nb}_{2} \mathrm{O}_{6}$ from pair distribution function analysis. Phys. Rev. B 2019, 99, 104102

[63] Enderby JE, Williams WG, Chapon LC, Hannon AC, Radaelli PG and Soper AK. GEM: 
The general materials diffractometers at ISIS - multibank capabilities for studying crystalline and disordered materials Neutron News 2004, 15, 19-

[64] Hannon AC. Results on disordered materials from the GEneral Materials diffractometer Nucl. Instrum. Meth. A 2005, 551, 88-107

[65] Smith RI, Hull S, Tucker MG, Playford HY, McPhail DJ, Waller SR and Norberg ST. The upgraded Polaris powder diffractometer at the ISIS neutron source. Rev. Sci. Instrum. 2019, 90, 115101

[66] Hannon AC, Howells WS and Soper AK. ATLAS: A suite of programs for the analysis of time-of-flight neutron diffraction data from liquid and amorphous samples. IOP Conf. Ser. 1990, 107 193-211

[67] https://www.isis.stfc.ac.uk/Pages/Disordered-Materials-Software.aspx

[68] Bushnell-Wye G and Cernik RJ. The general purpose two-circle diffractometer on Station 9.1, Daresbury Laboratory. Rev. Sci. Instrum. 1992, 63, 999-1001

[69] Bowron DT, Newport RJ, Rainford BD, Saunders GA and Senin HB. EXAFS and x-ray structural studies of $\left(\mathrm{Tb}_{2} \mathrm{O}_{3}\right)_{0.26}\left(\mathrm{P}_{2} \mathrm{O}_{5}\right)_{0.74}$ metaphosphate glass. Phys. Rev. B 1995, 51 5739-5745

[70] Warren BE and Mavel G. Elimination of the Compton component in amorphous scattering. Rev. Sci. Instrum. 1965, 36, 196-197

[71] Bushnell-Wye G, Finney JL, Turner J, Huxley DW and Dore JC. The use of synchrotron x rays to exploit the Warren-Mavel fluorescence detection technique in studies of disordered systems Rev. Sci. Instrum. 1992, 63, 1153-1155

[72] Wicks JD, Börjesson L, Bushnell-Wye G, Howells WS and McGreevy RL. Structure and ionic conduction in $(\mathrm{AgI})_{x}\left(\mathrm{AgPO}_{3}\right)_{1-x}$ glasses. Phys. Rev. Lett. 1995, 74, 726-729

[73] Strong SL and Kaplow R. Elimination of incoherent $\mathrm{x}$ rays with diffracted beam monochromators. Rev. Sci. Instrum. 1966, 3\%, 1495-1496

[74] Ruland R. The separation of coherent and incoherent Compton X-ray scattering. Brit. J. Appl. Phys. 1964, 15, 1301-1307

[75] Thijsse BJ. The accuracy of experimental radial distribution functions for metallic glasses. J. Appl. Cryst. 1984, 17, 61-76

[76] Bushnell-Wye G, Finney JL and Wicks JD. Use of new image-plate techniques in synchrotron radiation studies of materials and processes. Nucl. Instrum. and Meth. in Phys. Res. B 1995, 97, 557-560

[77] Roberts MA, Finney JL and Bushnell-Wye G. Development of Curved Image-Plate Techniques for Studies of Powder Diffraction, Liquids and Amorphous Materials. Mater. Sc. Forum 1998, 278-281, 318-322

[78] Petkov V, Jeong I-K, Chung JS, Thorpe MF, Kycia S and Billinge SJL. High real-space resolution measurement of the local structure of $\mathrm{Ga}_{1-x} \mathrm{In}_{x}$ As using X-ray diffraction. Phys. Rev. Lett. 1999, 83, 4089-4092

[79] Jeong I-K, Mohiuddin-Jacobs F, Petkov V, Billinge SJL. Local structure of $\operatorname{In}_{x} \mathrm{Ga}_{1-x} \mathrm{As}$ semiconductor alloys by high-energy synchrotron X-ray diffraction. Phys. Rev. B 2001, 63, 205202

[80] Fitch AN. The high resolution powder diffraction beam line at ESRF. J. Res. Natl Inst. Stand. Technol. 2004, 109, 133-142

[81] Willmott PR, Meister D, Leake SJ, Lange M, Bergamaschi A, Böge M, Calvi M, Cancellieri C, Casati N, Cervellino A, Chen Q, David C, Flechsig U, Gozzo F, Henrich B, Jäggi-Spielmann S, Jakob B, Kalichava I, Karvinen P, Krempasky J, Lüdeke A, Lüscher R, Maag S, Quitmann C, Reinle-Schmitt ML, Schmidt T, Schmitt B, Streun A, Vartiainen I, Vitins M, Wang X and Wullschlegera R. The Materials Science beamline upgrade at the Swiss Light Source. J. Synchrotron Rad. 2013, 20, 667-682

[82] https://www.diamond.ac.uk/Instruments/Crystallography/I15-1.html

[83] https://www.aps.anl.gov/Beamlines/Directory/Details?beamline_id=16

[84] Chupas PJ, Qiu X, Hanson JC, Lee PL, Grey CP and Billinge SJL. Rapid-acquisition pair distribution function (RA-PDF) analysis. J. Appl. Cryst. 2003, 36, 1342-1347

[85] Chupas PJ, Chapman KW and Lee PL. Applications of an amorphous silicon-based area 
detector for high-resolution, high-sensitivity and fast time-resolved pair distribution function measurements. J. Appl. Cryst. 2007, 40, 463-470

[86] Juelsholt M, Christiansen TL and Jensen KMØ. Mechanisms for tungsten oxide nanoparticle formation in solvothermal synthesis: from polyoxometalates to crystalline materials. J. Phys. Chem C 2019, 123, 5110-5119

[87] Hammersley AP, Svensson SO, Thompson A, Graafsma H, Kvick A and Moy JP. Calibration and correction of distortions in 2D detector systems. Rev. Sci. Instrum. 1995, 66, 2729-2733

[88] Juhás P, Davis T, Farrow CL and Billinge SJL. PDFgetX3: a rapid and highly automatable program for processing powder diffraction data into total scattering pair distribution functions. J. Appl. Cryst. 2013, 46, 560-566

[89] Thomae SLJ, Prinz N, Hartmann T, Teck M, Correll S and Zobel M. Pushing data quality for laboratory pair distribution function experiments. Rev. Sci. Instrum. 2019, 90, 043905

[90] Tucker MG, Keen DA, Dove MT and Trachenko K. Refinement of the Si-O-Si bond angle distribution in vitreous silica J. Phys. Condensed Matter 2005, 17, S67-S75

[91] Metropolis N, Rosenbluth AW, Rosenbluth MN, Teller AH and Teller E. Equation of state calculation by fast computing machines. J. Chem. Phys. 1953, 21, 1087-1092

[92] Nield VM, Keen DA, Hates W and McGreevy RL. Structural changes in silver bromide at the melting point. J. Phys.: Condensed Matter 1992, 4, 6703-6714

[93] Keen DA. Disordering phenomena in superionic conductors. J. Phys.: Condensed Matter 2002, 14, R819-R857

[94] Nield VM, Keen DA, Hayes W and McGreevy RL. Structure and fast-ion conduction in $\alpha$-AgI. Sol. State Ionics 1993, 66, 247-258

[95] Nield VM, McGreevy RL, Keen DA and Hayes W. Structural disorder in CuBr. Physica B 1994, 202, 159-166

[96] Keen DA and Hull S. Determination of structural disorder in superionic $\mathrm{Ag}_{2}$ Te by neutron total scattering. J. Phys.: Condensed Matter 1998, 10, 8217-8234

[97] Hull S, Norberg ST, Tucker MG, Eriksson SG, Mohn CE and Stølen S. Neutron total scattering study of the $\delta$ and $\beta$ phases of $\mathrm{Bi}_{2} \mathrm{O}_{3}$. Dalton Trans. 2009, 2009, 8737-8745

[98] Larson AC and Von Dreele RB. General Structure Analysis System (GSAS) Los Alamos National Laboratory, Report LAUR 86-748. 2000

[99] Toby BH. EXPGUI, a graphical user interface for GSAS. J. Appl. Cryst. 2001, 34, 210213

[100] Tucker MG, Dove MT and Keen DA. Application of the reverse Monte Carlo method to crystalline materials. J. Appl. Cryst. 2001, 34, 630-638

[101] Tucker MG, Dove MT and Keen DA. Simultaneous analysis of changes in long-range and short-range structural order at the displacive phase transition in quartz. J. Phys.: Condensed Matter 2000, 12, L723-L730

[102] Tucker MG, Keen DA and Dove MT. A detailed structural characterization of quartz on heating through the $\alpha-\beta$ phase transition. Miner. Mag. 2001, 65, 489-507

[103] Pawley GS. Unit-cell refinement from powder diffraction scans. J. Appl. Cryst. 1981, $14,357-361$

[104] Keen DA. Refining disordered structural models using reverse Monte Carlo methods: application to vitreous silica. Phase Transitions 1997, 61, 109-124

[105] Haines J, Cambon O, Keen DA, Tucker MG and Dove MT. Structural disorder and loss of piezoelectric properties in $\alpha$-quartz at high temperature. Appl. Phys. Lett. 2002, 81, 2968-2970

[106] Dove MT, Tucker MG and Keen DA. Neutron total scattering method: simultaneous determination of long-range and short-range order in disordered materials. Eur. J. Mineral. 2002, 14, 331-348

[107] Tucker MG, Goodwin AL, Dove MT, Keen DA, Wells SA and Evans JSO. Negative thermal expansion in $\mathrm{ZrW}_{2} \mathrm{O}_{8}$ : mechanisms, rigid unit modes and neutron total scattering. Phys. Rev. Lett. 2005, 95, 255501

[108] Tucker MG, Keen DA, Evans JSO and Dove MT. Local structure in $\mathrm{ZrW}_{2} \mathrm{O}_{8}$ from 
neutron total scattering. J. Phys.: Condensed Matter 2007, 19, 335215

[109] Evans JSO, David WIF and Sleight AW. Structural investigation of the negative-thermalexpansion material $\mathrm{ZrW}_{2} \mathrm{O}_{8}$. Acta Cryst. B 1999, 55, 333-340

[110] Pryde AKA, Hammonds KD, Dove MT, Heine V, Gale JD and Warren MC. Origin of the negative thermal expansion in $\mathrm{ZrW}_{2} \mathrm{O}_{8}$ and $\mathrm{ZrV}_{2} \mathrm{O}_{7}$. J. Phys.: Condensed Matter 1996, 8, 10973-10982

[111] Cao D, Bridges F, Kowach GR and Ramirez AP. Correlated atomic motions in the negative thermal expansion material $\mathrm{ZrW}_{2} \mathrm{O}_{8}$ : A local structure study Phys. Rev. B 2003, 68, 014303

[112] Conterio MJ, Goodwin AL, Tucker MG, Keen DA, Dove MT, Peters L and Evans JSO. Local structure in $\mathrm{Ag}_{3}\left[\mathrm{Co}(\mathrm{CN})_{6}\right]$ : Colossal thermal expansion, rigid unit modes and argentophilic interactions. J. Phys.: Condensed Matter 2008, 20, 255225

[113] Goodwin AL, Calleja M, Conterio MJ, Dove MT, Evans JSO, Keen DA, Peters L and Tucker MG. Colossal positive and negative thermal expansion in the framework material $\mathrm{Ag}_{3}\left[\mathrm{Co}(\mathrm{CN})_{6}\right]$. Science 2008, 319, 794-797

[114] Keen DA, Dove MT, Evans JSO, Goodwin AL, Peters L and Tucker MG. Hydrogenbonding transition and isotope-dependent negative thermal expansion in $\mathrm{H}_{3} \mathrm{Co}(\mathrm{CN})_{6}$. $J$. Phys.: Condensed Matter 2010, 22, 404202

[115] Pauling L and Pauling P. A trireticulate crystal structure: trihydrogen cobalticyanide and trisilver cobalticyanide. Proc. Nat. Acad. Sci. 1968, 60, 362-367

[116] http://scarc.library.oregonstate.edu/coll/pauling/rnb/46/rnb46.html

[117] Malarski Z, Sobczyk L and Grech E. Structure and IR spectroscopic behaviour of NHN hydrogen bonds. J. Mol. Structure 1988, 177, 339-349

[118] Proffen Th and Billinge SJL. PDFFIT, a program for full profile structural refinement of the atomic pair distribution function. J. Appl. Cryst. 1999, 32, 572-575

[119] Proffen Th, DiFrancesco RG, Billinge SJL, Brosha EL and Kwei GH. Measurement of the local Jahn-Teller distortion in $\mathrm{LaMnO}_{3.006}$. Phys. Rev. B 1999, 60, 9973-9977

[120] Qiu X, Proffen Th, Mitchell JF and Billinge SJL. Orbital correlations in the pseudocubic $O$ and rhombohedral $R$ phases of $\mathrm{LaMnO}_{3}$ Phys. Rev. Lett. 2005, 94, 177203

[121] Hou D, Zhao C, Paterson AR, Li S, Jones JL. Local structures of perovskite dielectrics and ferroelectrics via pair distribution function analyses. J. Eur. Ceram. Soc. 2018, 38, 971-987

[122] Halpern O and Johnson MH. On the magnetic scattering of neutrons. Phys. Rev. 1939, $55,898-923$

[123] Shull CG and Smart JS. Detection of antiferromagnetism by neutron diffraction. Phys. Rev. 1949, 76, 1256-1257

[124] Shull CG, Strauser WA and Wollan EO. Neutron diffraction by paramagnetic and antiferromagnetic substances. Phys. Rev. 1951, 83, 333-345

[125] Stewart JR, Andersen KH, Cywinski R and Murani AP. Magnetic diffuse scattering in disordered systems studied by neutron polarization analysis (invited). J. Appl. Phys. 2000, 87, 5425-5430

[126] Goodwin AL, Tucker MG, Dove MT and Keen DA. Magnetic structure of MnO at $10 \mathrm{~K}$ from total neutron scattering data. Phys. Rev. Lett. 2006, 96, 047209

[127] Blech IA and Averbach BL. Spin correlations in MnO. Physics 1964, 1, 31-44

[128] Paddison JAM, Gutmann MJ, Stewart JR, Tucker MG, Dove MT, Keen DA and Goodwin AL. The magnetic structure of paramagnetic MnO. Phys. Rev. B 2018, 97, 014429

[129] Keen DA and McGreevy RL. Determination of disordered magnetic structures by RMC modelling of neutron diffraction data. J. Phys.: Condensed Matter 1991, 3, 7383-7394

[130] Hannon AC, Wright AC and Sinclair RN. The atomic and magnetic structure of meltspun amorphous $\mathrm{Dy}_{7} \mathrm{Ni}_{3}$. Mater. Sci. \& Eng. A 1991, 134, 883-887

[131] Keen DA, Bewley RI, Cywinski R and McGreevy RL. Spin configurations in an amorphous random-anisotropy magnet. Phys. Rev. B 1996, 54, 1036

[132] Keffer F and O'Sullivan W. Problem of spin arrangements in MnO and similar antiferromagnets. Phys. Rev. 1957, 108, 637-644 
[133] Shirane G. A note on the magnetic intensities of powder neutron diffraction. Acta Cryst. 1959, 12, 282-285

[134] Shaked H, Faber, Jr J and Hitterman RL. Low-temperature magnetic structure of MnO: A high-resolution neutron-diffraction study. Phys. Rev. B 1988, 38, 11901-11903

[135] Paddison JAM, Stewart JR and Goodwin AL. spinvert: a program for refinement of paramagnetic diffuse scattering data. J. Phys.: Condensed Matter 2013, 25, 454220

[136] Paddison JAM and Goodwin AL. Empirical magnetic structure solution of frustrated spin systems. Phys. Rev. Lett. 2012, 108, 017204

[137] Frandsen BA, Yang X and Billinge SJL. Magnetic pair distribution function analysis of local magnetic correlations. Acta Cryst. A 2014, 70, 3-11

[138] Frandsen BA and Billinge SJL. Magnetic structure determination from the magnetic pair distribution function (mPDF): ground state of MnO. Acta Cryst. A 2015, 71, 325-334

[139] Juhás P, Davis T, Farrow CL and Billinge SJL. PDFgetX3: a rapid and highly automatable program for processing powder diffraction data into total scattering pair distribution functions J. Appl. Cryst. 2013, 46, 560-566

[140] Juhás P, Louwen JN, van Eijck L, Vogt ETC and Billinge SJL. PDFgetN3: atomic pair distribution functions from neutron powder diffraction data using ad hoc corrections $J$. Appl. Cryst. 2018, 51, 1492-1497

[141] Culbertson CM, Flak AT, Yatskin M, Cheong PH-Y, Cann DP and Dolgos MR. Neutron total scattering studies of group II titanates $\left(\mathrm{ATiO}_{3}, \mathrm{~A}^{2+}=\mathrm{Mg}, \mathrm{Ca}, \mathrm{Sr}, \mathrm{Ba}\right)$. Sci. Rep. 2020, 10, 3729

[142] Lager GA, Downs RT‘ Origlieri M abd Garoutte R. High-pressure single-crystal X-ray diffraction study of katoite hydrogarnet: Evidence for a phase transition from $I a 3 d \rightarrow I \overline{4} 3 d$ symmetry at 5 GPa Am. Miner. 2002, 87, 642-647

[143] Keen DA, Keeble DS and Bennett TD. Neutron and X-ray total scattering study of hydrogen disorder in fully hydrated hydrogrossular, $\mathrm{Ca}_{3} \mathrm{Al}_{2}\left(\mathrm{O}_{4} \mathrm{H}_{4}\right)_{3}$. Phys. Chem. Minerals, 2018, 45, 333-342

[144] Thygesen PMM, Young CA, Beake EOR, Romero FD, Connor LD, Proffen TE, Phillips AE, Tucker MG, Hayward MA, Keen DA and Goodwin AL. Local structure study of the orbital order/disorder transition in $\mathrm{LaMnO}_{3}$ Phys. Rev. B, 2017, 95, 174107

[145] Zhou J-S and Goodenough JB. Paramagnetic phase in single-crystal $\mathrm{LaMnO}_{3}$. Phys. Rev. B, 1999, 60, R15002

[146] Ahmed MR and Gehring GA. Volume collapse in $\mathrm{LaMnO}_{3}$ studied using an anisotropic Potts model. Phys. Rev. B, 2009, 79, 174106

[147] Eremenko M, Krayzman V, Bosak A, Playford HY, Chapman KW, Woicik JC, Ravel B and Levin I. Local atomic order and hierarchical polar nanoregions in a classical relaxor ferroelectric. Nature Comm. 2019, 10, 2728

[148] Senn MS, Keen DA, Lucas TCA, Hriljac JA and Goodwin AL. Emergence of long-range order in $\mathrm{BaTiO}_{3}$ from local symmetry-breaking distortions. Phys. Rev. Lett. 2016, 116, 207602

[149] Levin I, Krayzman V and Woicik JC. Local structure in perovskite (Ba,Sr) $\mathrm{TiO}_{3}$ : Reverse Monte Carlo refinements from multiple measurement techniques. Phys. Rev. B, 2014, 89, 024106

[150] Zhang N, Yokota H, Glazer AM, Ren Z, Keen DA, Keeble DS, Thomas PA and Ye Z-G. The Missing Boundary in the Phase Diagram of $\mathrm{PbZr}_{1-x} \mathrm{Ti}_{x} \mathrm{O}_{3}$. Nature Comm. 2014, 5, 5231

[151] Keeble DS, Barney ER, Keen DA, Tucker MG, Kreisel J and Thomas PA. Bifurcated polarization rotation in bismuth-based piezoelectrics. Adv. Funct. Mater. 2013, 23, 185190

[152] Comès R, Lambert M and Guinier A. Désordre linéaire dans les cristaux (cas du silicium, du quartz, et des pérovskites ferroélectriques). Acta Cryst. A 1970, 26, 244-254

[153] Keen DA and Dove MT. Local structures of amorphous and crystalline phases of silica, $\mathrm{SiO}_{2}$, by neutron total scattering. J. Phys.: Condensed Matter 1999, 11, 9263-9273

[154] Keen DA, Goodwin AL, Tucker MG, Dove MT and Evans JSO. Structural description 
of pressure-induced amorphization in $\mathrm{ZrW}_{2} \mathrm{O}_{8}$. Phys. Rev. Lett. 2007, 98, 225501

[155] Keen DA, Goodwin AL, Tucker MG, Hriljac JA, Bennett TD, Dove MT, Kleppe AK, Jephcoat AP and Brunellli M. Diffraction study of pressure-amorphized $\mathrm{ZrW}_{2} \mathrm{O}_{8}$ using in situ and recovered samples. Phys. Rev. B, 2011, 83, 064109

[156] Bennett TD, Goodwin AL, Dove MT, Keen DA, Tucker MG, Barney ER, Soper AK, Bithell EG, Tan J-C and Cheetham AK. Structure and properties of an amorphous metalorganic framework. Phys. Rev. Lett. 2010, 104, 115503

[157] Gaillac R, Pullumbi P, Beyer KA, Chapman KW, Keen DA, Bennett TD and Coudert FX. Liquid metal-organic frameworks. Nature Mater. 2017, 16, 1149-1155

[158] Bennett TD, Yue Y, Li P, Qiao A, Tao H, Greaves NG, Richards T, Lampronti GI, Redfern SAT, Blanc F, Farha OK, Hupp JT, Cheetham AK and Keen DA. Melt-quenched glasses of metal-organic frameworks. J. Am. Chem. Soc. 2016, 138, 3484-3492

[159] Keen DA and Bennett TD. Structural investigations of amorphous metal-organic frameworks formed via different routes. Phys. Chem. Chem. Phys. 2018, 20, 7857-7861

[160] Patterson AL. The Scherrer formula for X-ray particle size determination. Phys. Rev. 1939, 56, 978-982

[161] Ingham B. X-ray characterisation of nanoparticles. Cryst. Reviews 2015, 21, 229-303

[162] Christiansen TL, Cooper SR and Jensen KMØ. There's no place like real-space: elucidating size-dependent atomic structure of nanomaterials using pair distribution function analysis. Nanoscale Adv. 2020, 2, 2234-2254

[163] Korsounski VI, Neder RB, Hradil K, Barglik-Chory Ch, Müller G and Neuefeind J. Investigation of nanocrystalline $\mathrm{CdS}-$ glutathione particles by radial distribution function. J. Appl. Cryst. 2003, 36, 1389-1396

[164] Korsunskiy VI and Neder RB Exact model calculations of the total radial distribution functions for the X-ray diffraction case and systems of complicated chemical composition. J. Appl. Cryst. 2005, 38, 1020-1027

[165] Korsunskiy VI, Neder RB, Hofmann A, Dembski S, Graf C and Rühl E. Aspects of the modelling of the radial distribution function for small nanoparticles. J. Appl. Cryst. 2007, 40, 975-985

[166] Page K, Hood TC, Proffen Th and Neder RB. Building and refining complete nanoparticle structures with total scattering data. J. Appl. Cryst. 2011, 44, 327-336

[167] Proffen Th and Neder RB. DISCUS: a program for diffuse scattering and defect-structure simulation J. Appl. Cryst. 1997, 30, 171-175

[168] Neder RB and Proffen Th. Diffuse scattering and defect structure simulations: a cook book using program DISCUS. IUCr Texts on Crystallography, No 11. Oxford University Press (Oxford, UK.) 2008

[169] Bertolotti F, Moscheni D, Migliori A, Zacchini S, Cervellino A, Guagliardi A, Masciocchi N. A total scattering Debye function analysis study of faulted Pt nanocrystals embedded in a porous matrix. Acta Cryst. A 2016, 72, 632-644

[170] Jensen KM O, Juhas P, Tofanelli MA, Heinecke CL, Vaughan G, Ackerson CJ and Billinge SJL. Polymorphism in magic-sized $\mathrm{Au}_{144}(\mathrm{SR})_{60}$ clusters. Nature Comm. 2015, 7, 11859

[171] Lopez-Acevedo O, Akola J, Whetten RL, Grönbeck H and Häkkinen H. Structure and bonding in the ubiquitous icosahedral metallic gold cluster $\mathrm{Au}_{144}(\mathrm{SR})_{60}$. J. Phys. Chem. C 2009, 113 5035-5038

[172] Bahena D, Bhattarai N, Santiago U, Tlahuice A, Ponce A, Bach SBH, Yoon B, Whetten RL, Landman U and Jose-Yacaman M. STEM electron diffraction and high-resolution images used in the determination of the crystal structure of the $\mathrm{Au}_{144}(\mathrm{SR})_{60}$ cluster. $J$. Phys. Chem. Lett. 2013, 4, 975-981

[173] Zhang H and Banfield JF. Thermodynamic analysis of phase stability of nanocrystalline titania J. Mater. Chem. 1998, 8, 2073-2076

[174] Krayzman V, Cockayne E, Johnston-Peck AC, Vaughan G, Zhang F, Allen AJ, Kunz LY, Cargnello M, Friedman LH and Levin I. Local structural distortions and failure of the surface-stress "core-shell" model in brookite titania nanorods. Chem. Mater. 2020, 32, 
$286-298$

[175] Eremenko M, Krayzman V, Gagin A and Levin I. Advancing reverse Monte Carlo structure refinements to the nanoscale. J. Appl. Cryst. 2017, 50, 1561-1570

[176] Levin I, Keeble DS, Cibin G, Playford HY, Eremenko M, Krayzman V, Laws WJ and Reaney IM. Nanoscale polar heterogeneities and branching bi-displacement directions in $\mathrm{K}_{0.5} \mathrm{Bi}_{0.5} \mathrm{TiO}_{3}$. Chem. Mater. 2019, 31, 2450-2458

[177] Temkin RJ, Paul W and Connell GAN. Amorphous germanium II. Structural properties Adv. in Phys. 1973, 22, 581-641

[178] Cargill III GS. Influence of neglected small-angle scattering in radial distribution function analysis J. Appl. Cryst. 1971, 4, 277-283

[179] Farrow CL and Billinge SJL. Relationship between the atomic pair distribution function and small-angle scattering: implications for modeling of nanoparticles Acta Cryst. A $\mathbf{2 0 0 9}$, $65,232-239$

[180] Bowron DT, Soper AK, Jones K, Ansell S, Birch S, Norris J, Perrott L, Riedel D, Rhodes NJ, Wakefield SR, Botti A, Ricci M-A and Grazzi F. NIMROD: The Near and InterMediate Range Order Diffractometer of the ISIS second target station Rev. Sci. Instrum. 2010, 81, 033905

[181] Pinnavaia TJ, Pauly TR and Kim SS. Mesoporous molecular sieve catalysts: relationships between reactivity and long range structural order/disorder. In "Supported catalysts and their applications." Eds Sherrington DC and Kybett AP, Royal Society of Chemistry, Cambridge, UK (2001) pp19-26.

[182] Coelho AA. TOPAS and TOPAS-Academic: an optimization program integrating computer algebra and crystallographic objects written in C++ J. Appl. Cryst. 2018, 51, 210-218 (see also examples in https://community.dur.ac.uk/john.evans/topas_workshop/topas_user_menu.htm)

[183] Toby BH and Von Dreele RB. GSAS-II: the genesis of a modern open-source all purpose crystallography software package. J. Appl. Cryst. 2013, 46, 544-549

[184] https://www.mail-archive.com/gsas-ii@aps.anl.gov/msg00111.html

[185] Krayzman V, Levin I, Woicik JC and Bridges F. Correlated rattling-ion origins of dielectric properties in reentrant dipole glasses $\mathrm{BaTiO}_{3}-\mathrm{BiScO}_{3}$. Appl. Phys. Lett. 2015, 10\%, 192903

[186] Beecher AN, Semonin OE, Skelton JM, Frost JM, Terban MW, Zhai H, Alatas A, Owen JS, Walsh A and Billinge SJL. Direct observation of dynamic symmetry breaking above room temperature in methylammonium lead iodide perovskite. ACS Energy Lett. 2016, 1, 880-887

[187] Sanchez S, Steiner U and Hua X. Phase evolution during perovskite formation-insight from pair distribution function analysis Chem. Mater. 2019, 31, 3498-3506

[188] Hua X, Liu Z, Fischer MG, Borkiewicz O, Chupas PJ, Chapman KW, Steiner U, Bruce PG and Gey CP. Lithiation thermodynamics and kinetics of the $\mathrm{TiO}_{2}$ (B) nanoparticles J. Am. Chem. Soc. 2017, 139, 13330-13341

[189] Bozin ES, Yin WG, Koch RJ, Abeykoon M, Hor YS, Zheng H, Lei HC, Petrovic C, Mitchell JF and Billinge SJL. Local orbital degeneracy lifting as a precursor to an orbitalselective Peierls transition. Nature Comm. 2019, 10, 3638

[190] Koch RJ, Konstantinova T, Abeykoon M, Wang A, Petrovic C, Zhu Y, Bozin ES and Billinge SJL. Room temperature local nematicity in FeSe superconductor Phys. Rev. B 2019, 100, 020501(R)

[191] Keen DA and Goodwin AL. The crystallography of correlated disorder. Nature 2015 , 521, 303-309

[192] Cliffe MJ, Wan W, Zou X, Chater PA, Kleppe AK, Tucker MJ, Wilhelm H, Funnell NP, Coudert F-X and Goodwin AL. Correlated defect nanoregions in a metal-organic framework. Nature Comm. 2014, 5, 4176

[193] Cheetham AK, Bennett TD, Coudert F-X and Goodwin AL. Defects and disorder in metal organic frameworks. Dalton Trans. 2016, 45, 4113-4126

[194] Paddison JAM, Jacobsen H, Petrenko OA, Fernádez-Diaz MT, Deen PP and Good- 
win AL. Hidden order in spin-liquid $\mathrm{Gd}_{3} \mathrm{Ga}_{5} \mathrm{O}_{12}$. Science 2015, 350, 179-181

[195] Van Benschoten AH, Liu L, Gonzalez A, Brewster AS, Sauter NK, Fraser JS and Wall ME. Measuring and modeling diffuse scattering in protein X-ray crystallography. Proc. Natl Acad. Sci. 2016, 113, 4069-4074

[196] Wall ME, Wolff AM and Fraser JS. Bringing diffuse X-ray scattering into focus. Curr. Opinion in Struct. Bio. 2018, 50, 109-116

[197] Weber T and Simonov A. The three-dimensional pair distribution function analysis of disordered single crystals: basic concepts. Z. Kristallogr. 2012, 227, 238-247

[198] Simonov A, Weber T and Steurer W. Yell: a computer program for diffuse scattering analysis via three-dimensional delta pair distribution function refinement. J. Appl. Cryst. 2014, 47, 1146-1152

[199] Keen DA, Harris MJ and David WIF. Neutron time-of-flight measurements of diffuse scattering. Physica B 1998, 241-243, 201-203

[200] Simonov A, De Baerdemaeker T, Boström HLB, Ríos Gómez ML, Gray HJ, Chernyshov D, Bosak A, Bürgi H-B and Goodwin AL. Hidden diversity of vacancy networks in Prussian blue analogues Nature 2020, 578, 256-260

[201] Roth N, May AF, Ye F, Chakoumakos BC and Iversen BB. Model-free reconstruction of magnetic correlations in frustrated magnets IUCrJ 2018, 5, 410-416

[202] Keen DA and McGreevy RL. Structural modelling of glasses using reverse Monte Carlo simulation. Nature 1990, 344, 423-425 